The Astrophysical Journal Supplement Series, 165:400-431, 2006 July

(C) 2006. The American Astronomical Society. All rights reserved. Printed in U.S.A.

\title{
10,000 STANDARD SOLAR MODELS: A MONTE CARLO SIMULATION
}

\author{
JOHN N. BAHCALL ${ }^{1}$ \\ Institute for Advanced Study, Einstein Drive, Princeton, NJ 08540 \\ Aldo M. Serenelli \\ Institute for Advanced Study, Einstein Drive, Princeton, NJ 08540; and Max-Planck-Institut für Astrophysik, \\ Karl-Schwarzschild-Strasse 1, 85748 Garching, Germany \\ AND \\ SARBANi Basu \\ Department of Astronomy, Yale University, New Haven, CT 06520-8101 \\ Received 2005 November 18; accepted 2006 March 7
}

\begin{abstract}
We have evolved 10,000 solar models using 21 input parameters that are randomly drawn for each model from separate probability distributions for every parameter. We use the results of these models to determine the theoretical uncertainties in the predicted surface helium abundance, the profile of the sound speed versus radius, the profile of the density versus radius, the depth of the solar convective zone, the eight principal solar neutrino fluxes, and the fractions of nuclear reactions that occur in the CNO cycle or in the three branches of the $p$ - $p$ chains. We also determine the correlation coefficients of the neutrino fluxes for use in analysis of solar neutrino oscillations. Our calculations include the most accurate available input parameters, including radiative opacity, equation of state, and nuclear cross sections. We incorporate both the recently determined heavy element abundances recommended by Asplund et al. and the older (higher) heavy element abundances recommended by Grevesse \& Sauval. We present best estimates of many characteristics of the standard solar model for both sets of recommended heavy element compositions.
\end{abstract}

Subject headings: neutrinos — nuclear reactions, nucleosynthesis, abundances — Sun: abundances —

Sun: helioseismology — Sun: interior

\section{INTRODUCTION}

The primary purpose of this paper is to provide a quantitative basis for deciding if a given prediction from solar models agrees or disagrees with a measured value. We proceed by constructing solar models in which, for every model separately, each of 21 input parameters is drawn randomly from a corresponding probability distribution that describes our knowledge of the parameter. We evolve models with many different sets of input parameters and use the calculated distributions of different theoretical quantities to describe the statistical significance of comparisons between solar model predictions and helioseismological or neutrino measurements. To give an explicit example, the calculated probability distribution of the surface helium abundance is determined by evolving many different solar models, each with its own set of 21 randomly chosen input parameters, and counting how many solar models yield helium abundances within each specified bin or range of values.

The exquisite precision that has been obtained in helioseismology over the past decade and the revolutionary advances in understanding the properties of solar neutrinos make it appropriate to develop the best possible analysis techniques. New and more powerful measurements of helioseismological parameters and of solar neutrinos will be available in the next decade. The Monte Carlo simulations described in this paper will help to position us to take full advantage of the new data.

To the best of our knowledge, the calculations described in this paper are the first systematic attempt to use Monte Carlo simula-

\footnotetext{
1 John N. Bahcall passed away on 2005 August 17. He will be deeply missed.
}

tions to determine the uncertainties in solar model predictions of parameters measured by helioseismology. The helioseismological parameters we study are the depth of the convective zone, the surface helium abundance, and the profiles of the sound speed and density versus radius. Bahcall \& Ulrich (1988) used a less extensive Monte Carlo simulation, 1000 solar models and 5 input parameters, to determine the principal uncertainties in solar neutrino predictions. The Monte Carlo simulations described in the present paper provide a quantitative statistical basis for deciding if solar model predictions agree, or disagree, with helioseismological measurements. We do not know of any other statistical measure of the agreement, or lack of it, between solar models and helioseismology. As astroseismology continues to develop, Monte Carlo simulations of the kind described in this paper will be necessary to determine the statistical measure of agreement between stellar models and astroseismological measurements.

We provide in this paper the first full determination of the correlation coefficients of the predicted solar model neutrino fluxes, including correlations imposed by the evolution of the solar model as well as correlations introduced by specific input parameters. Previous discussions of correlations between neutrino fluxes have mostly been based on power-law approximations to the dependence of individual neutrino fluxes on specific input parameters (Fogli \& Lisi 1995; Fogli et al. 2002). The correlation coefficients determined here will make possible a simpler and somewhat more powerful analysis of solar neutrino propagation.

In subsequent papers, we will use the models calculated for this paper to discuss the uncertainties in quantities that require more extensive analysis to derive standard deviations. Examples of the quantities that will be studied later include the shapes 
TABLE 1

Best Estimates and $1 \sigma$ Uncertainties for 10 Important InPut Parameters FOR SOLAR MODELS

\begin{tabular}{|c|c|c|c|}
\hline $\begin{array}{l}\text { Quantity } \\
\text { (1) }\end{array}$ & $\begin{array}{c}\text { Best Estimate } \\
\text { (2) }\end{array}$ & $\begin{array}{c}1 \sigma \text { Uncertainty } \\
(\%) \\
(3)\end{array}$ & $\begin{array}{c}\text { Reference } \\
\text { (4) }\end{array}$ \\
\hline$p-p$ & $3.94 \times 10^{-25} \mathrm{MeV} \mathrm{b}$ & 0.4 & 1 \\
\hline${ }^{3} \mathrm{He}+{ }^{3} \mathrm{He} \ldots \ldots \ldots \ldots \ldots$. & $5.4 \mathrm{MeV} \mathrm{b}$ & 6.0 & 2,3 \\
\hline${ }^{3} \mathrm{He}+{ }^{4} \mathrm{He} . \ldots \ldots \ldots \ldots \ldots . . . . .$. & $0.53 \mathrm{keV} \mathrm{b}$ & 9.4 & 3,4 \\
\hline${ }^{7} \mathrm{Be}+e^{-} \ldots \ldots \ldots \ldots \ldots$ & Eq. (26), ref. 3 & 2 & 3,5 \\
\hline${ }^{7} \mathrm{Be}+p \ldots \ldots \ldots \ldots \ldots$. & $20.6 \mathrm{eV} \mathrm{b}$ & 3.8 & 6 \\
\hline 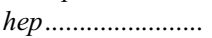 & $8.6 \times 10^{-20} \mathrm{keV} \mathrm{b}$ & 15.1 & 1 \\
\hline${ }^{14} \mathrm{~N}+p \ldots \ldots \ldots \ldots \ldots \ldots$ & $1.69 \mathrm{keV} \mathrm{b}$ & 8.4 & 7,8 \\
\hline age ............................ & $4.57 \times 10^{9} \mathrm{yr}$ & 0.44 & 9 \\
\hline diffusion ............... & 1.0 & 15.0 & 10 \\
\hline luminosity.............. & $3.8418 \times 10^{33}$ ergs s$^{-1}$ & 0.4 & 11,12 \\
\hline
\end{tabular}

Notes.- Some comments on the input parameters are given in the text in $\S 2.1$. The first seven quantities listed in col. (1) of the table refer to the rates of the lowenergy nuclear fusion reactions. The last three quantities represent the current age of the Sun, the element diffusion coefficient, and the present-day luminosity of the Sun measured with photons. The best estimate of 1.0 for the diffusion coefficient corresponds to the value calculated by Thoul et al. (1994). The second and third columns give, respectively, the best estimate of each of the quantities and the $1 \sigma$ uncertainty, expressed in percent of the best estimate.

References.- - (1) Park et al. (2003); (2) Junker et al. (1998); (3) Adelberger et al. (1998); (4) Singh et al. (2004); (5) Gruzinov \& Bahcall (1997); (6) Junghans et al. (2003); (7) Formicola et al. (2004); (8) Runkle et al. (2005); (9) Bahcall \& Pinsonneault (1995); (10) Thoul et al. (1994); (11) Fröhlich \& Lean (1998); (12) Bahcall et al. (2005a).

of the production probabilities versus radius for solar neutrino fluxes, the shape of the electron distribution versus radius, and the shape of the neutron distribution versus radius. These three quantities are all necessary for a precise analysis of solar neutrino oscillations.

At present, the uncertainties in the heavy element abundances on the surface of the Sun represent the dominant uncertainties in the prediction with solar models of many quantities of interest. We therefore carry out simulations using three different choices for the heavy element abundances and their uncertainties (see discussion below). We use the same uncertainties for noncomposition parameters for all three choices of the heavy element abundances. Table 1 lists the uncertainties in 10 important input parameters; $\S 3$ discusses the uncertainties due to radiative opacity and equation of state.

Studies of the theoretical uncertainties in standard solar models have been the subject of numerous studies in the past. In the spirit of the present work, the investigation by Bahcall \& Ulrich (1988) represents the most complete study previously published. Some other works with a similar aim (the list is not extensive) are those of Turck-Chièze et al. (1988), Sackmann et al. (1990), Guenther et al. (1992, 1996), Turck-Chièze \& Lopes (1993), Boothroyd \& Sackmann (2003), Couvidat et al. (2003), and Guzik et al. (2005).

\subsection{The Dilemma Posed by the Heavy Element Abundances}

New and much improved determinations for volatile elements have led to lower estimated photospheric abundances for the very important elements C, N, O, Ne, and Ar (see Asplund et al. 2000, 2004, 2005; Lodders 2003; Allende Prieto et al. 2001, 2002). Reductions range between 0.13 and 0.24 dex. The photospheric abundance of Si has also been reported (Asplund 2000) to be smaller than previous determinations by $0.05 \mathrm{dex}$. Si is usually used as reference element to link the photospheric and meteoritic abundance scales (Lodders 2003). As a result, a lower value of the photospheric $\mathrm{Si}$ leads to an equal reduction of the meteoritic abundances of other important elements (e.g., $\mathrm{Mg}, \mathrm{S}, \mathrm{Ca}$, Fe, Ni).

These new determinations use three-dimensional calculations (not one-dimensional as in the previous calculations), which solve the MHD equations consistently with radiative transfer and which correctly predict observed line widths. Moreover, the new calculations frequently include non-LTE effects; observational effects such as blends are treated carefully. The net result is that for the new calculations the abundances inferred from molecular and atomic lines are generally in agreement, whereas this was often not the case in previous abundance studies.

Surprisingly, these new (lower) heavy element abundances, when included in solar model calculations, lead to best-estimate predictions for helioseismologically measured quantities like the depth of the convective zone, the surface helium abundance, and the radial distributions of sound speeds and densities that are in strong disagreement with the helioseismological measurements (Bahcall \& Pinsonneault 2004, hereafter BP04; Bahcall et al. 2005a, 2005c; Basu \& Antia 2004). So far there has not been a successful resolution of this problem (see, e.g., BP04; Bahcall et al. 2004b, 2005c; Basu \& Antia 2004; Antia \& Basu 2005; Turck-Chièze et al. 2004; Guzik \& Watson 2004; Guzik et al. 2005; Seaton \& Badnell 2004; Badnell et al. 2005; Montalbán et al. 2004).

Given what we know about the input parameters of the solar models, are the disagreements between solar models that incorporate the new abundances (as summarized in Asplund et al. 2005, hereafter AGS05 abundances) and helioseismological measurements statistically significant? And, if so, at what significance level? The Monte Carlo calculations described in this paper are required to answer these questions.

Quite remarkably, the older (higher) heavy element abundances (as summarized in Grevesse \& Sauval 1998, hereafter GS98 abundances) lead to good agreement with helioseismological measurements when incorporated into precise solar models (see, e.g., Bahcall et al. 2001b, 2005c; BP04; Basu \& Antia 2004). In this subject, for now, it seems that "Better is worse."

With this unclear situation regarding heavy element abundances, what is our best strategy to simulate the uncertainties in the surface chemical composition? We hedge our bets. We simulate 5000 solar models for both of the following cases: (1) adopt AGS05 abundances using the perhaps "optimistic" uncertainties determined by Asplund et al. (2005) and summarized in Table 3 of this paper (hereafter AGS05-Opt composition choice); and (2) adopt GS98 recommended abundances but with "conservative" uncertainties given in Table 3 of the this paper (hereafter GS98-Cons composition choice). These two cases represent our primary Monte Carlo simulation. In addition, we compute 1000 solar models for a third hybrid case: (3) adopt the newer AGS05 abundances but with conservative uncertainties; we denote this option AGS05-Cons.

\subsection{How This Paper Was Written}

Our greatest fear in carrying out this project was that we would discover something that we wanted to change after we had calculated the 10,000 Monte Carlo models. In order to avoid this disaster, we went carefully over all the details by examining the outputs of many sets of small numbers of models (10-100) that were ultimately discarded, but which we used to refine the technical details of how we handled the simulations of input parameters and the calculations and analysis of solar models.

Based on the preliminary calculations, we wrote a complete draft of the paper that described all the technical details and the 
results (including tables and figures). We decided we needed to complete this exercise before running the 10,000 models to make sure that the results were understandable and self-consistent and that the simulations were indeed doing what we wanted them to do. Although this is an unorthodox way to write a paper, it turned out to be essential for this project.

We discovered using the preliminary models that we had to adjust some important technical aspects of our simulation. For example, we had to shift the mean of the lognormal distribution of the simulated heavy element composition variables so as to give the observed best estimate composition value (see eq. [6]). We had to make several adjustments in the size and distribution of the mesh points in our final models so as to make possible robust and automated helioseismological inversions. As we wrote up the results, we realized that there were additional things that we needed to print out and analyze or save. A paper that is written in this unusual way should probably be read in an unusual way (see $\oint \oint 1.4-1.6)$.

\subsection{Outline of This Paper}

We present in $\S 2$ the best estimates and $1 \sigma$ uncertainties for 19 of the 21 input parameters, including all 7 of the critical nuclear parameters, as well as the solar age and luminosity, the diffusion coefficient, and, perhaps more importantly, the nine most significant heavy element abundances. The equation of state and the radiative opacities are treated separately in $\S 3$. In this section we describe how we compute the effective $1 \sigma$ uncertainties for the radiative opacity and the equation of state for all of the measurable quantities that we calculate with solar models. We also give in this section the computed $1 \sigma$ uncertainties due to opacity and equation of state for all of the predicted solar model quantities. We then describe in $\S 4$ the stellar evolution code used in the calculations and some numerical issues, particularly regarding the precision with which each solar model has been calculated in order that the numerical error for every model is less than $0.1 \sigma$ of the estimated uncertainty in each of the calculated helioseismological and neutrino predictions. In $\S 5$ we present and discuss the best estimate predictions of our standard (preferred) solar models for 23 output parameters. We also present in this section the best estimates for the production profile versus radius of each solar neutrino flux and the profiles of the electron and neutron number densities. We present in $\S 6$ our Monte Carlo results for the depth of the solar convective zone and for the surface helium abundance. We also compare these results with the helioseismologically measured values. Section 7 compares the calculated solar model sound speed profiles and the density profiles with the results of helioseismological measurements. We describe in $\S 8$ the Monte Carlo results for the distributions of individual solar neutrino fluxes and also illustrate the important correlations between the different fluxes. In $\S 9$, we tabulate and discuss the correlation coefficients among the predicted neutrino fluxes. We present and discuss in $\S 10$ the fractions of the total solar nuclear energy generation that occur via different fusion pathways. Finally, we summarize our main results and discuss their implications in $\S 11$.

\subsection{How Should This Paper Be Read?}

We think most readers will be product oriented. They will want to see the results and will not be as interested in the technical details of how the calculations were done. We describe the technical details in this paper; they are necessary in order for the experts to evaluate our results and may be useful in other contexts. But we do not expect anyone but dedicated experts to read these descriptions.
Therefore, most readers should begin by leafing through the paper to get a general impression of what is included, paying particular attention to figures and tables. Very few readers need to go through the paper in the logical order in which it is written.

\subsection{What to Skip}

The average reader can easily skip essential aspects of our presentation like the choice of the 19 best estimate values for the input parameters that are given in $\S 2$ and the technical way that we simulate composition uncertainties (also described in $\S 2$ ). To use the results, it is also not necessary to understand how we have evaluated uncertainties due to the input functions that represent the radiative opacity and the equation of state $(\S 3)$. Only aficionados of solar modeling will be interested in $\S 4$ on the precision with which we have calculated different parameters and technical details like the number of radial shells used in the evaluations.

\subsection{What to Read}

We give here some examples of sections that may be of interest to readers with expertise in different areas. If you teach a course that touches on solar energy or on stellar evolution, or if you are an astronomer working in a specialty not connected to stellar evolution or to the Sun, you may find it interesting to peruse the section on the standard solar model, $\S 5$. This perusal will give you a feel for what we can calculate about the Sun. Then you can jump to the final summary and discussion, $\S 11$, to get an overall picture of the agreement between the solar model and different experiments and to appreciate the outstanding challenges.

If you are interested in helioseismology or astroseismology, you will want to look the results given in $\S \S 6$ and 7 . In these sections, we present the uncertainties in predicting quantities that have been measured helioseismologically: the distribution of sound speeds, the distribution of the matter density, the surface abundance of helium, and the depth of the convective zone. We also compare the measured and predicted values for helioseismological variables and discuss the extent to which the theoretical and observed values agree or disagree.

If you are interested in neutrinos, you will want to look carefully at the results presented in $\S 8$. We describe in this section the uncertainties in the predicted neutrino fluxes and compare the best-fit values with the inferences from solar neutrino experiments. We also describe the correlations that are potentially observable between the $p e p, p-p$, and ${ }^{7} \mathrm{Be}$ solar neutrino fluxes. In $\S 9$ the correlation coefficients between all the computed neutrino fluxes are given. In addition, we describe in $\S 5.4$ the distribution of the production probability of each of the neutrino fluxes, and in $\S 5.5$ we present the electron and neutron number densities versus radius (quantities that are required to discuss aspects of neutrino oscillations).

Of course, stellar model theorists may be interested in some of the technical details regarding the calculation of our solar models, details that are given in $\S 4$ (brief description of the stellar evolution code and precision of the models) and $\S 5$ (input parameters and their accuracy).

Nuclear astrophysicists may like to know the fraction of solar energy generation that takes place in different reaction paths. This information is given in Table 18 of $\S 10$. Nuclear physicists in particular may find it useful to look at Table 1 to see the current status of the most important nuclear fusion cross sections and the discussion in $\S 8$ to understand how the nuclear uncertainties affect the predicted neutrino fluxes.

We hope that most readers will be interested in the conclusions and discussion presented in $\S 11$. 


\section{BEST ESTIMATES AND UNCERTAINTIES FOR INPUT PARAMETERS}

In this section, we present the best estimate, or standard, values we adopt for each of the input parameters of the solar models. We also present the $1 \sigma$ uncertainties of the best estimate parameters.

In $\S 2.1$, we tabulate and discuss for 10 important input parameters the best-fit values and $1 \sigma$ uncertainties. These $10 \mathrm{pa}-$ rameters include all the critical nuclear parameters as well as the solar age and luminosity and the diffusion coefficient for heavy element diffusion. In $\S 2.2$, we describe for the nine most important surface heavy element abundances the best estimate values we adopt and their associated uncertainties. The simulation of the composition uncertainties is less straightforward than the simulation of the uncertainties for the 10 noncomposition parameters discussed in $\S 2.1$. We present in $\S 2.3$ the equations that are used to simulate the composition uncertainties. We describe in $\S 2.4$ how the software works that produces the 19 simulated input parameters discussed above for each solar model.

The final 2 input parameters that we consider, out of a total of 21 , are the radiative opacity and the equation of state. The opacity and equation of state are complicated functions, unlike the parameters discussed in this section, which are all scalar numbers. Therefore, we defer a discussion of the radiative opacity and the equation of state to a separate discussion in $\S 3$.

\subsection{Ten Important Input Parameters}

Table 1 presents the best estimates and the associated $1 \sigma$ uncertainties that we have adopted for each of 10 important input parameters to the solar models. The most recent references on which we rely for these data are given in the last column of the table.

The reader may find useful some brief comments regarding Table 1. The first seven rows of the table refer, with the exception of the row for the ${ }^{7} \mathrm{Be}+e^{-}$reaction, to the low-energy cross section factors for the indicated nuclear fusion reactions (see for example chap. 3 of Bahcall 1989). The entries for the $p-p$ reaction (low-energy cross section factor $S_{11}$ ) and the hep reaction have recently been recalculated with a rather high precision (Park et al. 2003) culminating more than six decades of theoretical work on the $p$ - $p$ reaction. The ${ }^{3} \mathrm{He}-{ }^{3} \mathrm{He}$ reaction $\left(S_{3,3}\right)$ has been measured, in an experimental tour de force, down to the energies at which solar fusion occurs (Junker et al. 1998).

The rate of the ${ }^{3} \mathrm{He}\left({ }^{4} \mathrm{He}, \gamma\right){ }^{7} \mathrm{Be}$ reaction $\left(S_{3,4}\right)$ represents the most important nuclear physics uncertainty in the prediction of solar neutrino fluxes (see BP04). We continue to use the estimated uncertainty given by Adelberger et al. (1998). However, a recent measurement by Singh et al. (2004) gives a best estimate that agrees exactly with the Adelberger et al. recommended value but with a much smaller error bar. The important resulting Singh et al. measurement should be checked by other experimental groups before it can be used to reduce the error estimate for the ${ }^{3} \mathrm{He}+{ }^{4} \mathrm{He}$ reaction. The measurements should also be extended to lower energies; the Singh measurement goes down to $420 \mathrm{keV}$.

The reaction ${ }^{7} \mathrm{Be}\left(e^{-}, \nu\right)^{7} \mathrm{Li}$ is, unlike the other nuclear reactions listed in Table 1, an electron capture reaction, not a nucleonnucleon fusion reaction. The electron is attracted to the ${ }^{7} \mathrm{Be}$ nucleus, not repelled by Coulomb forces as in a nucleon-nucleon reaction. Therefore, the ${ }^{7} \mathrm{Be}+e^{-}$reaction cannot be described by a low-energy cross section factor in the way that nucleonnucleon fusion reactions are described. The reaction rate must be calculated theoretically, not measured. We use formula (26) of Adelberger et al. (1998) for the rate of the ${ }^{7} \mathrm{Be}+e^{-}$reaction. This formula has a coefficient that is about $1 \%$ higher than was obtained in the previous theoretical calculations that go back more than 40 years. The reason is that Adelberger et al. (1998) use the recalculation by Bahcall (1994) of the capture rate from states of ${ }^{7} \mathrm{Be}$ that are bound in the Sun. In his recalculation, Bahcall used profiles of the temperature, density, and chemical composition obtained from modern solar models.

In recent years, reevaluations of the rate of the ${ }^{14} \mathrm{~N}(p, \gamma){ }^{15} \mathrm{O}$ reaction have yielded values much smaller for the astrophysical factor $S_{1,14}$ than the previous adopted value (Angulo \& Descouvemont 2001; Mukhamedzhanov et al. 2003). More importantly, the reaction rate has been measured recently by two beautiful, independent experiments (Formicola et al. 2004; Runkle et al. 2005). We use the weighted average cross section $\left(S_{1,14}\right)$ obtained from the measurements of Formicola and Runkle for this reaction and the associated $1 \sigma$ uncertainty. The rates of other important nuclear reactions not listed in Table 1 are taken from Adelberger et al. (1998).

G. Wasserburg determined the "age of the Sun" to be bounded by $4.563 \times 10^{9} \mathrm{yr}<t_{\odot}<4.576 \times 10^{9} \mathrm{yr}$ (see Appendix in Bahcall $\&$ Pinsonneault 1995). We adopt the central value of this interval as the solar age and the interval limits to represent $\pm 1 \sigma$ uncertainties. There is some uncertainty regarding to what stage of the pre-main-sequence solar evolution this age represents. Premain-sequence evolution of a $1 M_{\odot}$ stellar model lasts about (3-4) $\times 10^{7} \mathrm{yr}$. We adopt one-half of the central value of the premain-sequence lifetime as a measure $(1 \sigma)$ of the systematic uncertainty regarding the formation time of meteorites. The final uncertainty in the solar age quoted in Table 1 is the quadratic combination of both uncertainties mentioned above. A more thorough discussion can be found in Bahcall \& Pinsonneault (1995). The solar luminosity is the same as adopted in Bahcall et al. (2005a), and its uncertainty is discussed in Bahcall \& Pinsonneault (1995).

We use the diffusion subroutine that is described in Thoul et al. (1994) and that is publicly available online. ${ }^{2}$ Our best estimate for the diffusion rate assumes that the results from this subroutine are exactly correct (hence, the best estimate value of 1.0 in Table 1 ). A discussion of the adopted uncertainty is also given in Thoul et al. (1994; see also Proffitt 1994).

\subsection{Composition Parameters}

In recent years, determinations of the solar abundances of heavy elements have become more refined and detailed (Lodders 2003 and especially Asplund 2000; Asplund et al. 2000, 2004, 2005; Allende Prieto et al. 2001, 2002). These recent determinations yield significantly lower values than were previously adopted (e.g., by Grevesse \& Sauval 1998) for the abundances of the volatile heavy elements: $\mathrm{C}, \mathrm{N}, \mathrm{O}, \mathrm{Ne}$, and Ar. However, these recent abundance determinations lead to solar models that disagree with helioseismological measurements (BP04; Basu \& Antia 2004). By contrast, solar models that use the older determinations of element abundances by Grevesse \& Sauval (1998) are in excellent agreement with helioseismology (BP04; Bahcall et al. 2005c; Basu \& Antia 2004; Antia \& Basu 2005; TurckChièze et al. 2004; Guzik et al. 2005; Montalbán et al. 2004).

As of this writing, we do not know the reason for the discrepancy between helioseismological measurements and the predictions of solar models constructed with the more recently determined heavy element abundances. We therefore carry out independent simulations using the older heavy element abundances

\footnotetext{
2 See http://www.sns.ias.edu/ jnb.
} 
TABLE 2

Adopted Abundances

\begin{tabular}{|c|c|c|}
\hline $\begin{array}{c}\text { Element } \\
\text { (1) }\end{array}$ & $\begin{array}{c}\text { GS98 } \\
(2)\end{array}$ & $\begin{array}{c}\text { AGS05 } \\
\text { (3) }\end{array}$ \\
\hline $\mathrm{C} \ldots .$. & 8.52 & 8.39 \\
\hline N... & 7.92 & 7.78 \\
\hline $\mathrm{O} \ldots \ldots$ & 8.83 & 8.66 \\
\hline $\mathrm{Ne} \ldots .$. & 8.08 & 7.84 \\
\hline $\mathrm{Na} \ldots .$. & 6.32 & 6.27 \\
\hline $\operatorname{Mg} \ldots$. & 7.58 & 7.53 \\
\hline Al......... & 6.49 & 6.43 \\
\hline Si & 7.56 & 7.51 \\
\hline $\mathrm{S}$ & 7.20 & 7.16 \\
\hline $\mathrm{Ar}$ & 6.40 & 6.18 \\
\hline $\mathrm{Ca} \ldots \ldots \ldots \ldots$ & 6.35 & 6.29 \\
\hline 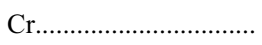 & 5.69 & 5.63 \\
\hline $\mathrm{Mn}$ & 5.53 & 5.47 \\
\hline 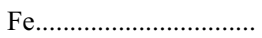 & 7.50 & 7.45 \\
\hline 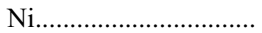 & 6.25 & 6.19 \\
\hline
\end{tabular}

NoTES.-Best estimate element abundances for the two abundance compilations adopted in this work (Grevesse \& Sauval 1998, GS98) and (Asplund et al. 2005, AGS05). Only elements accounted for in the radiative opacity calculations by the Opacity Project group are given.

recommended by Grevesse \& Sauval (1998) and the more recent heavy element abundances recommended by Asplund et al. (2005). In Table 2 we summarize the best estimate values for the GS98 and AGS05 compositions adopted in this paper. Only elements accounted for in the Opacity Project radiative opacity calculations are given (Badnell et al. 2005).

We follow the compilers of heavy element abundances in regarding as the appropriate quantity on which to focus attention the logarithmic ratio

$$
\text { abundance }_{i}=\log \left(N_{i} / N_{\mathrm{H}}\right)+12.0 .
$$

The quantity abundance is $_{i}$ the logarithmic ratio of the number of atoms of type $i$ divided by the number of hydrogen atoms $\left(N_{\mathrm{H}}\right)$ on the scale in which the logarithm of the number of hydrogen atoms is 12.0 .

We vary the heavy element abundances for the following nine important elements: C, N, O, Ne, Mg, Si, S, Ar, and Fe. We have carried out numerical experiments with different solar models to verify that the nine heavy elements considered here are overwhelmingly the most significant for solar modeling. The remaining elements listed in Table 2, i.e., $\mathrm{Na}, \mathrm{Al}, \mathrm{Ca}, \mathrm{Cr}, \mathrm{Mn}$, and $\mathrm{Ni}$, are kept equal to their best estimate value in the Monte Carlo simulations.

We define in the next two subsections abundance uncertainties that we caricature as "conservative" uncertainties and "optimistic" uncertainties.

\subsubsection{Conservative Uncertainties}

We first define "conservative [historical] uncertainties" (see col. [2] of Table 4 of Bahcall \& Serenelli 2005). We calculate conservative uncertainties by assuming that the differences between the Asplund et al. (2005) recommended abundances and the Grevesse \& Sauval (1998) recommended abundances represent the current $1 \sigma$ uncertainties. Thus,

$$
\sigma\left(\text { abundance }_{i}\right)=\mid \text { abundance }_{i}\left(\text { GS98) } - \text { abundance } _ { i } \left(\text { AGSO5) }_{\text {bu }}\right.\right.
$$

TABLE 3

\begin{tabular}{|c|c|c|}
\hline $\begin{array}{l}\text { Heavy Element } \\
\text { (1) }\end{array}$ & $\begin{array}{l}\text { Conservative (Historical) } \\
\text { (dex) } \\
\text { (2) }\end{array}$ & $\begin{array}{c}\text { Optimistic } \\
\text { (Asplund et al. 2005) } \\
\text { (dex) } \\
\text { (3) }\end{array}$ \\
\hline С & 0.13 & 0.05 \\
\hline $\mathrm{N}$ & 0.14 & 0.06 \\
\hline $\mathrm{O}$ & 0.17 & 0.05 \\
\hline $\mathrm{Ne}$ & 0.24 & 0.06 \\
\hline $\mathrm{Mg}$ & 0.05 & 0.03 \\
\hline 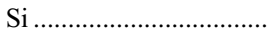 & 0.05 & 0.02 \\
\hline 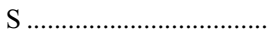 & 0.04 & 0.04 \\
\hline $\mathrm{Ar}$ & 0.22 & 0.08 \\
\hline 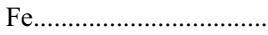 & 0.05 & 0.03 \\
\hline
\end{tabular}

Adopted $1 \sigma$ Uncertainties for Individual Heavy Elements (in dex)

Notes.-We give in col. (2), under the heading "Conservative," our preferred estimated errors, the differences between the recent abundance determinations (Asplund et al. 2005) and the previously standard values (Grevesse \& Sauval 1998) (see eq. [2]). Col. (3), under the heading "Asplund et al. 2005," lists our "optimistic uncertainties"; these uncertainties are quoted in the recent paper by (Asplund et al. 2005). We use meteoritic abundances and uncertainties for the nonvolatile elements $\mathrm{Mg}, \mathrm{Si}, \mathrm{S}$, and $\mathrm{Fe}$.

where in equation (2) GS98 stands for the composition recommended by Grevesse \& Sauval (1998) and AGS05 stands for the composition recommended by Asplund et al. (2005).

\subsubsection{Optimistic Uncertainties}

The primary uncertainties in the determination of heavy element abundances are generally not the measurement errors. The most important uncertainties are usually the systematic uncertainties that arise from the detailed modeling of the solar atmosphere that is necessary in order to infer element abundances from the measurements of line strengths. It is very difficult to assess the systematic uncertainties that arise from the modeling. We cite as evidence of this difficulty the fact that when compilers of element abundances list errors they usually do not specify whether they intend their errors to be used as $1 \sigma$ uncertainties, $3 \sigma$ uncertainties, or to have some other significance.

We define here as "optimistic $1 \sigma$ uncertainties" the abundance uncertainties recommended by Asplund et al. (2005). We use the characterization "optimistic" in contrast to the "conservative" uncertainties defined in $\S 2.2 .1$. The optimistic uncertainties are a factor of 2 or more smaller than the conservative uncertainties for the most abundant elements (see Table 3).

\subsection{Simulating Composition Uncertainties}

We describe in this subsection how we simulate the distribution of uncertainties for each of the heavy element abundances. This question deserves special attention since people working in the field of element abundances almost universally quote best estimates and uncertainties in terms of logarithms of the number abundances. Since symmetric logarithmic uncertainties result in asymmetric errors on the abundances $\left(\left[10^{+\epsilon}-1.0\right]\right.$ is different from $\left.\left|10^{-\epsilon}-1.0\right|\right)$, special care must be taken to make sure that logarithmic uncertainties translate into uncertainties for the abundances that have the desired properties (e.g., the correct average value).

Let

$$
y=\log _{10}\left[\left(N_{i} / N_{\mathrm{H}}\right) /\left(N_{i} / N_{\mathrm{H}}\right)_{0}\right],
$$

where $\log \left(N_{i} / N_{\mathrm{H}}\right)_{0}$ is the tabulated (recommended) value of the abundance. Let $\sigma$ be the uncertainty in $\log \left(N_{i} / N_{\mathrm{H}}\right)_{0}$ that is listed 
in Table 3. We assume a normal distribution for $y$ with the tabulated value of $\sigma$ (in dex). Thus,

$$
P(y) d y=[\sqrt{(2 \pi) \sigma}]^{-1} \exp \left[-y^{2} /\left(2 * \sigma^{2}\right)\right] d y .
$$

The normal distribution in the logarithm of $\left(N_{i} / N_{\mathrm{H}}\right) /\left(N_{i} / N_{\mathrm{H}}\right)_{0}$ translates into a lognormal distribution of $\left(N_{i} / N_{\mathrm{H}}\right) /\left(N_{i} / N_{\mathrm{H}}\right)_{0}$. This translation is exhibited by letting

$$
z \equiv \frac{\left(N_{i} / N_{\mathrm{H}}\right)}{\left(N_{i} / N_{\mathrm{H}}\right)_{0}} .
$$

Then

$$
P(z)=\left[z \sqrt{(2 \pi) \sigma_{1}}\right]^{-1} \exp \left[-(\ln z)^{2} /\left(2 * \sigma_{1}^{2}\right)\right],
$$

where $\sigma_{1}$ is $\ln 10 \sigma$. The variable $z$ is lognormal distributed with an average value

$$
\langle z\rangle=\exp \left(\sigma_{1}^{2} / 2\right)
$$

We want $\langle z\rangle$ to be equal to 1.0. To accomplish this, we shift the whole distribution by considering instead of $z$ the variable $z^{\prime}$, where

$$
z^{\prime} \equiv z-\left[\exp \left(\sigma_{1}^{2} / 2\right)-1.0\right] \equiv \frac{\left(N_{i} / N_{\mathrm{H}}\right)^{\prime}}{\left(N_{i} / N_{\mathrm{H}}\right)_{0}}
$$

Then, because of the relation between the average and the standard deviation in a lognormal distribution, equation (7), we have $\left\langle z^{\prime}\right\rangle=1.0$.

We calculate $z^{\prime}$, which is used in the stellar evolution program and in evaluating opacities, from equations (5) and (8). Thus,

$$
z^{\prime}=\frac{\left(N_{i} / N_{\mathrm{H}}\right)^{\prime}}{\left(N_{i} / N_{\mathrm{H}}\right)_{0}}=10^{y}-\left[\exp \left(\sigma_{1}^{2} / 2\right)-1.0\right]
$$

In general, the standard deviation of $z^{\prime}$ can be related to the standard deviation of $y$ by

$$
\sigma\left(z^{\prime}\right)=\sigma\left(10^{y}\right)=\sqrt{\exp \left(\sigma_{1}^{2}(y)\right)\left[\exp \left(\sigma_{1}^{2}(y)\right)-1\right]}
$$

where, as before, $\sigma_{1}(y)=\ln 10 \sigma(y)$. For small values if $10^{y}=$ $\left(N_{i} / N_{\mathrm{H}}\right) /\left(N_{i} / N_{\mathrm{H}}\right)_{0}$, they are related by a simple factor

$$
\sigma\left(z^{\prime}\right)=\sigma\left(10^{y}\right) \simeq \ln 10 \sigma(y) .
$$

\subsection{Simulation Software}

The essence of our Monte Carlo simulations is software that chooses for each solar model a randomly selected value for each of the 19 parameters discussed in this section. For each of the 10 parameters discussed in $\S 2.1$, the software chooses a particular value from a Gaussian probability distribution with the mean and standard deviation given in Table 1. For the nine composition variables, the software chooses for each solar model particular values from probability distributions with the uncertainties listed in Table 3 and, as appropriate, with the best estimate heavy element abundances as given in Grevesse \& Sauval (1998) or Asplund et al. (2005).

Since we consider very large numbers of models, 5000 in each simulation, there is a small chance that a simulated value for one of the variables will be nonphysical, if we accept without thinking the probability distributions discussed in $\S \S 2.1$, 2.2, and 2.3. The problematic cases could be the neon and argon composition variables when the conservative uncertainties are adopted, in which case a strict application of the lognormal probability distribution would yield a few models with neon or argon abundances less than zero due to the shift in mean value (eq. [8]). To deal with this situation, the software rejects nonphysical values, i.e., negative simulated values for positive definite quantities such as cross sections or compositions, and repeats the random selection until a positive value is found. Given that negative, nonphysical values of neon and argon are expected to occur at the 3.3 and $3.9 \sigma$ level, respectively, we do not expect this procedure will introduce any bias in the simulated data.

\section{UNCERTAINTIES DUE TO OPACITY AND EOS}

We describe in $\S 3.1$ how we compute the effective $1 \sigma$ uncertainties that arise from uncertainties in the radiative opacity and in the equation of state (EOS). Since these quantities are not single numbers like the input parameters discussed in $\S 2$, the estimate of the effective errors of the opacity and the EOS have to be computed separately for each output quantity of interest, depending on the sensitivity of each quantity to the radiative opacity and the EOS. We then present and discuss in $\S 3.2$ the calculated effective $1 \sigma$ uncertainties due to the radiative opacity, and in $\S 3.3$ we present the results for the $1 \sigma$ uncertainties due to the equation of state.

\subsection{Definition of Effective $1 \sigma$ Uncertainties for Opacity and Equation of State}

We begin this subsection by defining how we compute the uncertainties in different solar model predictions that are caused by our imperfect knowledge of the radiative opacity and the equation of state. Then we illustrate these definitions by showing explicitly how we calculate the uncertainties in the rms sound speed profile.

Let us denote by $X$ the solar model quantity for which we want to determine the uncertainty introduced by the opacity uncertainties. First, we evolve two solar models that are identical except that one model uses the recent OP opacity calculations (Badnell et al. 2005; Seaton \& Badnell 2004; Seaton 2005) and the other model uses the OPAL opacity (Iglesias \& Rogers 1996). From this pair of matched solar models we get two values for $X$ we call $X_{i}(\mathrm{OP})$ and $X_{i}(\mathrm{OPAL})$ for the models with the OP and the OPAL opacities, respectively (the subscript $i$ denotes a given pair of matched models). The unbiased estimator $s_{i}^{2}$ for the variance is

$$
s_{i}^{2}(X(\text { opacity }))=\frac{\left[X_{i}(\mathrm{OP})-X_{i}(\mathrm{OPAL})\right]^{2}}{2} .
$$

We define the fractional standard deviation squared

$\sigma_{i}^{2}(X($ opacity $))=\frac{s_{i}^{2}(X(\text { opacity }))}{\mu_{i}^{2}(X(\text { opacity }))}=2 \frac{\left[X_{i}(\mathrm{OP})-X_{i}(\mathrm{OPAL})\right]^{2}}{\left[X_{i}(\mathrm{OP})+X_{i}(\mathrm{OPAL})\right]^{2}}$,

where $\mu_{i}(X$ (opacity) $)$ is the mean value between $X_{i}(\mathrm{OP})$ and $X_{i}$ (OPAL).

In order to obtain a more representative value for $\sigma(X$ (opacity)), we decided to average the difference shown in equation (13) over a matched set of $N=20$ pairs of solar models. In all $N$ cases, one member of each pair of models was constructed using the OP 
opacity and one member was constructed using the OPAL opacity. For each pair of models, the 19 input parameters discussed in $\S 2$ were simulated as described in $\S 2.4$. The 19 parameters were the same for both members of each pair, but different parameters were simulated for all of the $N$ pairs. The OPAL 2001 equation of state was used in all cases.

In practice, we calculated $\sigma(X$ (opacity) $)$ from the equation

$$
\sigma(X(\text { opacity }))=\sqrt{N^{-1} \sum_{i} \sigma_{i}^{2}(X(\text { opacity }))}
$$

where as before $i$ denotes a pair of matched solar models (same values for the 19 input parameters discussed in $\S 2$ and EOS).

The effective $1 \sigma$ fractional uncertainty in $X$ due to the equation of state is calculated in an analogous fashion. In this case, the matched pairs of solar models are computed by changing only the EOS. We use the 2001 OPAL equation of state (Rogers 2001) and the earlier 1996 OPAL equation of state (Rogers et al. 1996). Thus,

$$
\sigma(X(\mathrm{EOS}))=\sqrt{N^{-1} \sum_{i} \sigma_{i}^{2}(X(\mathrm{EOS}))}
$$

where $\sigma_{i}^{2}(X(\mathrm{EOS}))$ for each pair of matched solar models is computed as

$$
\sigma_{i}^{2}(X(\mathrm{EOS}))=2 \frac{\left[X_{i}(\mathrm{EOS} 2001)-X_{i}(\mathrm{EOS} 1996)\right]^{2}}{\left[X_{i}(2001)+X_{i}(1996)\right]^{2}}
$$

One of the quantities that is of greatest interest is the distribution of sound speeds predicted by the solar model. We characterize this distribution by the root mean squared (rms) difference between the sound speeds predicted by a given solar model and the sound speeds inferred from the measured helioseismological frequencies. Thus,

$$
\delta c=\sqrt{M^{-1} \sum_{i=1}^{M}\left[\frac{\left(c_{\odot}-c_{\text {model }}\right)^{2}}{c_{\odot}^{2}}\right]},
$$

where the summation is carried out over $M$ shells in the solar model. For consistency and greatest accuracy, the inversion of the helioseismological frequencies to obtain the solar sound speeds is accomplished using as a reference model the same solar model whose sound speed is being considered (see, e.g., Basu et al. 2000). We define the rms difference in densities, $\delta \rho$, analogous to the definition of $\delta \mathrm{c}$ in equation (17) by

$$
\delta \rho=\sqrt{M^{-1} \sum_{i=1}^{M}\left[\frac{\left(\rho_{\odot}-\rho_{\text {model }}\right)^{2}}{\rho_{\odot}^{2}}\right]} .
$$

We perform the summation indicated in equations (17) and (18) over three separate regions: (1) the interior region: $0.07 R_{\odot} \leq$ $R \leq 0.45 R_{\odot} ;(2)$ the exterior region: $0.45 R_{\odot} \leq R \leq 0.95 R_{\odot}$; and (3) the entire measured region: $0.07 R_{\odot} \leq R \leq 0.95 R_{\odot}$. We have broken up the measured domains of sound speeds and of densities into these three regions because the region just below the solar convective zone is relatively poorly described by the standard solar models (see, e.g., Fig. 13 of Bahcall et al. 2001 b or Fig. 1 of Bahcall et al. 2005c).
We illustrate the use of equation (13) by showing explicitly how we calculate the effective $1 \sigma$ uncertainty of the sound speed distribution due to uncertainties in the radiative opacity. We have

$$
\sigma(\delta c(\text { opacity }))=\sqrt{N^{-1} \sum_{i} \sigma_{i}^{2}(\delta c(\text { opacity }))}
$$

where, analogously to equation (13),

$$
\sigma_{i}^{2}(\delta c(\text { opacity }))=2 \frac{\left[\delta c_{i}(\mathrm{OP})-\delta c_{i}(\mathrm{OPAL})\right]^{2}}{\left[\delta c_{i}(\mathrm{OP})+\delta c_{i}(\mathrm{OPAL})\right]^{2}} .
$$

Similarly, for the $1 \sigma$ uncertainty in $\delta c$ due to the equation of state uncertainties, we have

$$
\sigma(\delta c(\mathrm{EOS}))=\sqrt{N^{-1} \sum_{i} \sigma_{i}^{2}(\delta c(\mathrm{EOS}))}
$$

where for each individual pair of matched solar models (same 19 input parameters and radiative opacities),

$$
\sigma_{i}^{2}(\delta c(\mathrm{EOS}))=2 \frac{\left[\delta c_{i}(\mathrm{EOS} 2001)-\delta c_{i}(\mathrm{EOS} 1996)\right]^{2}}{\left[\delta c_{i}(\mathrm{EOS} 2001)+\delta c_{i}(\mathrm{EOS} 1996)\right]^{2}} .
$$

\subsection{Effective $1 \sigma$ Uncertainties due to Radiative Opacity}

In this subsection, we describe and discuss the effective $1 \sigma$ uncertainties due to the radiative opacity. Table 4 presents the effective $1 \sigma$ uncertainties due to radiative opacity for individual solar neutrino fluxes, measured helioseismological parameters, and the parameters that characterize the different nuclear fusion reactions that are responsible for solar energy generation. The results were calculated using equation (14). The numerical values without parentheses were computed using solar models that incorporate the Grevesse \& Sauval (1998) heavy element abundances; the values in parentheses were computed using the Asplund et al. (2005) abundances. The uncertainties are given in all cases in fractional percent.

For all the solar neutrino fluxes, the radiative opacity introduces errors that are small compared to the previously estimated total uncertainties in the predicted and the measured solar neutrino fluxes (BP04; Bahcall \& Serenelli 2005; Bahcall et al. 2004a). This statement is correct for solar models computed with both the Grevesse \& Sauval (1998) heavy element abundances, as well as the Asplund et al. (2005) abundances. However, the $\sim 2 \%(\sim 1 \%)$ uncertainty in the predicted ${ }^{8} \mathrm{~B}$ solar neutrino flux due to the radiative opacity is comparable to some of the other commonly calculated theoretical uncertainties for this important flux. Nevertheless, even for ${ }^{8} \mathrm{~B}$ neutrinos the radiative opacity contributes an uncertainty that is a factor of several below the total theoretical uncertainty for this important neutrino flux.

For the surface helium abundance and the depth of the convective zone, the radiative opacity contributes uncertainties that are comparable to the claimed accuracy in the helioseismological measurements. For the surface helium abundance, the quoted measurement error is 0.0034 or $1.4 \%$ (see eq. [24] and Basu \& Antia 2004), which should be compared with the smaller $0.3 \%$ uncertainty due to the radiative opacity (Table 4). For the depth of the convective zone, the spread among accurate measurements is about 0.001 or $0.14 \%$ (see eq. [23] and Basu \& Antia 2004; see also Kosovichev \& Fedorova 1991; Christensen-Dalsgaard et al. 
TABLE 4

$\sigma\left(\right.$ Opacity $\left._{\text {eff }}\right)$ : Effective Standard Deviations due to Uncertainties in the Radiative Opacity

\begin{tabular}{|c|c|c|c|c|c|}
\hline $\begin{array}{l}\text { Neutrino Flux } \\
\text { (1) }\end{array}$ & $\begin{array}{c}\text { Effective } 1 \sigma \\
(\%) \\
(2)\end{array}$ & $\begin{array}{l}\text { Helioseismological Quantity } \\
\text { (3) }\end{array}$ & $\begin{array}{c}\text { Effective } 1 \sigma \\
(\%) \\
(4)\end{array}$ & $\begin{array}{c}\text { Nuclear } \\
\text { Fusion } \\
\text { Branch } \\
(5)\end{array}$ & $\begin{array}{c}\text { Effective } 1 \sigma \\
(\%) \\
(6)\end{array}$ \\
\hline$p-p$ & $0.07(0.04)$ & $Y_{\text {surf }}$ & $0.32(0.29)$ & $p-p$ & $0.01(<0.01)$ \\
\hline 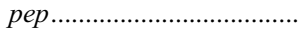 & $0.17(0.10)$ & $R_{\mathrm{cz}}$ & $0.17(0.10)$ & $\mathrm{CNO}$ & $1.29(0.97)$ \\
\hline hер & $0.23(0.18)$ & $\delta c_{\text {all }}$ & $29.0(12.6)$ & $p-p(\mathrm{I})$ & $0.10(0.07)$ \\
\hline${ }^{7} \mathrm{Be}$ & $0.78(0.62)$ & $\delta c_{\text {inner }}$ & $19.4(7.2)$ & $p-p(\mathrm{II})$ & $0.78(0.61)$ \\
\hline${ }^{8} \mathrm{~B}$ & $1.87(1.36)$ & $\delta c_{\text {outer }}$ & $32.0(13.5)$ & $p-p(\mathrm{III})$ & $0.79(0.61)$ \\
\hline${ }^{13} \mathrm{~N}$ & $1.14(0.86)$ & $\delta \rho_{\text {all }}$ & $26.8(8.7)$ & & \\
\hline${ }^{15} \mathrm{O}$ & $1.49(1.12)$ & $\delta \rho_{\text {inner }}$ & $17.4(13.3)$ & & \\
\hline${ }^{17} \mathrm{~F}$ & $1.65(1.24)$ & $\delta \rho_{\text {outer }}$ & $29.2(8.7)$ & & \\
\hline
\end{tabular}

Notes.-The standard deviations were computed with the aid of eq. (14), using solar models evolved separately for the OPAL and OP radiative opacity determinations. The values without parentheses were computed using solar models that incorporate the Grevesse \& Sauval (1998) heavy element abundances; the values in parentheses were computed using Asplund et al. (2005) abundances. The first two columns of the table refer to solar neutrino fluxes. The third and fourth columns give results for helioseismological quantities: the surface helium abundance, the depth of the convective zone, the rms difference between the solar sound speed and the model sound speed (for the total measured range; the inner region: $R \leq 0.45 R_{\odot}$; and the outer region: $R \geq 0.45 R_{\odot}$, see eqs. [17] and [19]), as well as the analogous rms differences between the solar density and the model density. The last two columns present results for percentages of the solar energy generation that involves different nuclear paths: all $p-p$ reactions; all CNO reactions; $p-p(\mathrm{I})$ (terminated by ${ }^{3} \mathrm{He}-{ }^{3} \mathrm{He}$ or $\left.\mathrm{p}+{ }^{2} \mathrm{H}\right) ; p-p(\mathrm{II})$ (terminated through $e^{-}+{ }^{7} \mathrm{Be}$ ) and $p$ - $p$ (III) (terminated through $p+{ }^{7} \mathrm{Be}$ ). The $1 \sigma$ uncertainty is given in percent of the relevant quantity.

1991), while the radiative opacity causes an uncertainty of $0.17 \%$ ( $0.10 \%$ for AGS05 abundances) that is comparable or larger ( Table 4).

The radiative opacity causes a huge uncertainty, $\sim 20 \%-32 \%$ ( $\sim 7 \%-14 \%$ for AGS05 heavy element abundances), in the calculated profile of the sound speed. The uncertainty in the density profile due to the radiative opacity varies from about $17 \%$ (13\% for AGS05 abundances) in the inner region $\left(R \leq 0.45 R_{\odot}\right)$ to $30 \%$ ( $\sim 9 \%$ for AGS05 abundances) in the outer region of the Sun $\left(R \geq 0.45 R_{\odot}\right)$.

The calculated fractions of the nuclear fusion reactions that take different paths in the Sun are practically independent of uncertainties due to the radiative opacity (last two columns of Table 4). In all cases, the fractional uncertainties are $\lesssim 1 \%$ in the frequencies that different nuclear fusion paths are taken, with the only exception being CNO for the GS98 composition, for which we get $1.3 \%$.

\subsection{Effective $1 \sigma$ Uncertainties due to Equation of State}

In this subsection, we present and discuss the calculated effective $1 \sigma$ uncertainties due to the equation of state. We determine the uncertainties for the EOS from equation (15).
Table 5 summarizes the effective uncertainties that are due to a lack of knowledge of the equation of state. We see immediately from Table 5 that the uncertainty in the EOS does not significantly affect the calculation of the neutrino fluxes (see col. [2] of Table 5) nor the fraction of the nuclear energy generation that occurs via different fusion pathways (see col. [6] of Table 5). For both the neutrino fluxes and the fusion fractions, the fractional uncertainties are in all cases less than $0.5 \%$.

Also, the surface helium abundance is only affected by $0.2 \%$, and the depth of the convective zone by less than $0.01 \%$, by the uncertainty in the EOS. Both of these uncertainties are small compared to the helioseismological measurement errors.

The situation is different for the sound speed profile and the density profile. For these profiles, the uncertainty in the equation of state can cause a $1 \sigma$ difference that ranges from about $12 \%(6 \%$ for AGS05 abundances) to $21 \%$ (15\% for AGS05 abundances), depending on whether one considers the sound or the density profile and whether one considers the total profile or the inner or outer profile.

\section{SOLAR MODEL CALCULATIONS}

In $\S 4.1$ we briefly describe the stellar evolution code used for computing the solar models of our Monte Carlo simulations. In

TABLE 5

$\sigma$ (Equation of State eff): Effective Fractional Standard Deviations due to Uncertainties in the Equation of State

\begin{tabular}{|c|c|c|c|c|c|}
\hline $\begin{array}{l}\text { Neutrino Flux } \\
\text { (1) }\end{array}$ & $\begin{array}{c}\text { Effective } 1 \sigma \\
(\%) \\
(2)\end{array}$ & $\begin{array}{l}\text { Helioseismological } \\
\text { Quantity } \\
\text { (3) }\end{array}$ & $\begin{array}{c}\text { Effective } 1 \sigma \\
(\%) \\
(4)\end{array}$ & $\begin{array}{l}\text { Nuclear } \\
\text { Fusion } \\
\text { Branch } \\
(5)\end{array}$ & $\begin{array}{c}\text { Effective } 1 \sigma \\
(\%) \\
(6)\end{array}$ \\
\hline$p-p$ & $0.02(0.02)$ & $Y_{\text {surf }}$ & $0.12(0.14)$ & $p-p$ & $0.00(<0.01)$ \\
\hline pep & $0.01(0.01)$ & $R_{\mathrm{cz}}$ & $<0.01(<0.01)$ & $\mathrm{CNO}$ & $0.22(0.24)$ \\
\hline hер & $0.05(0.05)$ & $\delta c_{\text {all }}$ & $11.6(5.2)$ & $p-p(\mathrm{I})$ & $0.02(0.02)$ \\
\hline${ }^{7} \mathrm{Be}$ & $0.18(0.20)$ & $\delta c_{\text {inner }}$ & $16.2(11.3)$ & $p-p(\mathrm{II})$ & $0.18(0.20)$ \\
\hline${ }^{8} \mathrm{~B}$ & $0.30(0.33)$ & $\delta c_{\text {outer }}$ & $13.7(4.6)$ & $p-p(\mathrm{III})$ & $0.18(0.20)$ \\
\hline${ }^{13} \mathrm{~N}$ & $0.20(0.21)$ & $\delta \rho_{\text {all }}$ & $15.7(4.2)$ & & \\
\hline${ }^{15} \mathrm{O}$ & $0.24(0.26)$ & $\delta \rho_{\text {inner }}$ & $10.4(13.0)$ & & \\
\hline${ }^{17} \mathrm{~F}$ & $0.26(0.29)$ & $\delta \rho_{\text {outer }}$ & $17.8(4.1)$ & & \\
\hline
\end{tabular}

Notes.-The standard deviations were computed with the aid of eq. (15), using solar models evolved separately for the OPAL 1996 and OPAL 2001 equations of state. The notation is the same as for Table 4. 
$\S 4.2$ we describe the precision with which the solar models were computed. In particular, we summarize the results of tests carried out using different numbers of radial zones, time steps, and criteria for convergence to the adopted solar luminosity, radius, and chemical composition.

\subsection{Stellar Evolution Code}

The stellar evolution code used for computing the solar models in our Monte Carlo simulations is the Garching stellar evolution code, which has been described in some detail in Weiss $\&$ Schlattl (2000) with the updates/modifications mentioned in Bahcall et al. (2005a). Crucial to this work is the calculation of appropriate radiative opacities that depend not only on the total metallicity assumed for the Sun but on the individual element abundances. For this reason, we compute for each solar model in our simulations a complete new set of radiative opacity tables corresponding to the simulated composition. This has been performed using the data and software tools provided by the Opacity Project group (Seaton 2005).

\subsection{Precision of Solar Model Calculations}

In general, we have set the numerical parameters of our stellar evolution code such that the errors we make in calculating the desired solar parameters - neutrino related quantities and helioseismological parameters - are less than $0.1 \sigma$ of the current uncertainty in predicting each parameter.

Our best standard solar models (Bahcall et al. 2005c) have approximately 2000 radial mesh points. The base of the convective zone is particularly well resolved by using a grid spacing $\Delta R / R_{\odot} \approx 4 \times 10^{-5}$ in a region centered at the base of the convective zone and extending by $0.002 R_{\odot}$ both outward and inward. Because the depth of the convective zone evolves very slowly during solar evolution, redistributing mesh points in each evolutionary step is enough to guarantee that the depth of the convective zone is very well defined at all times. This high density of mesh points near the boundary of the convective zone is necessary in order to compute a precise depth of the convective zone. Evolution from the zero-age main sequence to the solar age is accomplished with evolutionary time steps that are not longer than 10 Myr. Convergence of the model to the measured values of the solar luminosity, radius, and surface $Z / X$ is considered satisfactory when the relative differences between the computed and the adopted values are smaller than $10^{-6}$ for each of the three quantities. With these conditions, the computational time required to calculate a solar model is kept within reasonable limits if only a few solar models have to be computed; however, the computational time becomes prohibitively large when thousands of models are required.

The computational time can be reduced by relaxing the constraints on the model accuracy. However, when a less stringent convergence criterion is adopted, e.g., fewer mesh points are used, or a longer evolutionary time step is permitted, the solar model predictions deviate slightly from those of the more accurate models. As a practical compromise, we allow small deviations of the predicted solar model quantities from the results of our most precise models, deviations that are less than or equal to $0.1 \sigma$ of the current uncertainty in the predictions of each parameter. Among the quantities discussed in this paper, the predicted values that are most sensitive to the numerical accuracy of the solar models are, given their small current theoretical uncertainties, the depth of the convective zone and the $p-p$ and pep neutrino fluxes. The calculated depth of the convective zone is sensitive to the radial mesh density, while the neutrino fluxes are mostly affected by the evolutionary time step.

Guided by trial and error, we performed a series of numerical tests and found an acceptable set of constraints that preserves the desired accuracy while significantly reducing the required computational time. There are three important sets of requirements that we have used in evolving models for the Monte Carlo calculations discussed in this paper. First, the convergence accuracy is $10^{-4}$ in the solar radius, luminosity, and surface $Z / X$. Second, the total number of mesh points in each solar model is about 1200 during the initial 3.5 Gyr of evolution and is smoothly increased from that moment on until the model has about 1800 mesh points at the end of the evolution. At all times, the high mesh density near the base of the convective zone is same as in our most precise models. This fine mesh distribution is necessary for the solar sound speed and density inversions to have a similar level of accuracy as our best solar models described in $\S 5$. Third, evolutionary time steps of up to $15 \mathrm{Myr}$ (50\% longer than in our most precise models) are allowed.

The computational time is reduced by more than a factor of 3 relative to our standard models (see $\S 5$ ) for solar models computed with these precision requirements. However, the calculated values of all the neutrino fluxes and nuclear fusion rates and all of the helioseismological parameters we discuss in this paper are the same as in our most precisely calculated models to within an accuracy of $0.1 \sigma$ of the current theoretical uncertainty. In particular, the $p$ - $p$ and pep neutrino fluxes and the depth of the convective zone of our best standard solar models (Bahcall et al. $2005 \mathrm{c})$ are reproduced with the less precise models considered here to better than $0.07 \sigma(p-p), 0.05 \sigma(p e p)$, and $0.07 \sigma$ (convective zone). Other quantities have larger theoretical uncertainties and thus the errors introduced by using less accurate models become negligible. For example, for the important ${ }^{8} \mathrm{~B}$ neutrino flux the error due to the reduced requirements for the precision of the solar models is only $0.005 \sigma\left({ }^{8} \mathrm{~B}\right)$.

\section{THE STANDARD SOLAR MODEL}

We present in this section the best estimate predictions of our standard solar models. The most important input parameters, aside from composition variables, are listed in Table 1. Any input quantities not discussed explicitly in $\S 2$ are the same as described in Bahcall et al. (2005c), BP04, or Bahcall et al. (2001b), with the latest description taking precedence. The best estimate heavy element abundances are given in Grevesse \& Sauval (1998) and Asplund et al. (2005). For short, we will sometimes refer to these standard solar models as, respectively, the BSB(GS98) and the BSB(AGS05) standard models.

The only difference between the models discussed in this section and the models discussed in Bahcall et al. (2005c) is that for the models presented here (and throughout this paper) we use the improved low-temperature opacities of Ferguson et al. (2005) rather than the previously available opacities of Alexander \& Ferguson (1994). The improved low-temperature opacities make no significant difference in any of the quantities we consider here except for the depth of the convective zone. For the BS05(OP) model, the agreement with helioseismology is slightly improved by using the new opacities. The Ferguson et al. (2005) opacities decrease the depth of the convective zone by $0.07 \%$ (or $0.0005 R_{\odot}$ ) relative to the values obtained with the Alexander \& Ferguson (1994) values.

The free parameters in our solar models are the initial helium abundance $Y_{\text {init }}$, the initial metallicity $Z_{\text {init }}$ and the mixing length parameter $\alpha$. Our zero-age main-sequence model is a $1 M_{\odot}$ 
TABLE 6

Standard Solar Model Predictions: Measurable Quantities

\begin{tabular}{|c|c|c|c|c|c|}
\hline $\begin{array}{l}\text { Neutrino } \\
\text { Source } \\
\text { (1) }\end{array}$ & $\begin{array}{l}\text { Neutrino Flux } \\
\text { (2) }\end{array}$ & $\begin{array}{l}\text { Helioseismological } \\
\text { Quantity } \\
\text { (3) }\end{array}$ & $\begin{array}{l}\text { Helioseismological } \\
\text { Value } \\
\text { (4) }\end{array}$ & $\begin{array}{c}\text { Other } \\
\text { Quantities } \\
(5)\end{array}$ & $\begin{array}{l}\text { Calculated } \\
\text { Value } \\
(6)\end{array}$ \\
\hline$p-p \ldots \ldots$ & $5.99(6.06)$ & $Y_{\text {surface }}$ & $0.2426(0.2291)$ & $\mathrm{Cl}(\mathrm{SNU})$ & $8.12(6.58)$ \\
\hline рер & $1.42(1.45)$ & $R_{\mathrm{cz}}$ & $0.7132(0.7279)$ & $\mathrm{Ga}(\mathrm{SNU})$ & $126.08(118.88)$ \\
\hline hер & $7.93(8.25)$ & $\delta c_{\text {all }}$ & $0.00099(0.00488)$ & $p-p$ & $99.2 \%(99.5 \%)$ \\
\hline${ }^{7} \mathrm{Be}$ & $4.84(4.34)$ & $\delta c_{\text {inner }}$ & $0.00077(0.00239)$ & CNO & $0.78 \%(0.50 \%)$ \\
\hline${ }^{8} \mathrm{~B}$ & $5.69(4.51)$ & $\delta c_{\text {outer }}$ & $0.00114(0.00606)$ & $p-p(\mathrm{I})$ & $88.3 \%(89.6 \%)$ \\
\hline${ }^{13} \mathrm{~N}$ & $3.05(2.00)$ & $\delta \rho_{\text {all }}$ & $0.0113(0.0442)$ & $p-p($ II $)$ & $10.8 \%(9.6 \%)$ \\
\hline${ }^{15} \mathrm{O}$ & $2.31(1.44)$ & $\delta \rho_{\text {inner }}$ & $0.0054(0.0070)$ & $p-p(\mathrm{III})$ & $0.91 \%(0.81 \%)$ \\
\hline${ }^{17} \mathrm{~F}$ & $5.83(3.25)$ & $\delta \rho_{\text {outer }}$ & $0.0143(0.0591)$ & & \\
\hline
\end{tabular}

NoтеS.-The values without parentheses were calculated using the Grevesse \& Sauval (1998) heavy element abundances and represent our preferred model BSB(GS98). The values that are enclosed in parentheses were obtained with a solar model that uses the Asplund et al. (2005) solar heavy element abundances and represent the model BSB(AGS05). The table presents the predicted neutrino fluxes in the first two columns, in units of $10^{10}(p-p), 10^{9}\left({ }^{7} \mathrm{Be}\right), 10^{8}\left(p e p,{ }^{13} \mathrm{~N},{ }^{15} \mathrm{O}\right), 10^{6}\left({ }^{8} \mathrm{~B},{ }^{17} \mathrm{~F}\right)$, and $10^{3}(h e p) \mathrm{cm}^{-2} \mathrm{~s}^{-1}$. The third and fourth columns give the calculated quantities that are measured helioseismologically: the surface helium abundance, the depth of the convective zone, and the fractional uncertainties in the rms profiles $(\delta c$ and $\delta \rho)$ of the sound speed and density (all measured points, as well as the inner and outer regions of the Sun; see eq. [17] for a definition of the rms fractional differences). The last two columns give the solar model predictions, assuming no neutrino oscillations, for the chlorine and gallium solar neutrino experiments, and the percentage of nuclear fusion energy that is generated by different paths. The quantities $p-p$ and CNO refer, respectively, to the full collection of $p-p$ and CNO fusion reactions. The percentages for the different $p-p$ branches are denoted by $p-p(\mathrm{I}), p-p$ (II), and $p-p$ (III), respectively (see also the caption to Table 4).

homogeneous star. An acceptable solar model has to have the present-day solar luminosity, radius, and surface metallicity at the present solar age within a precision already discussed in $\S 4.2$.

\subsection{Predictions for 23 Measurable Quantities}

Table 6 gives, for 23 measurable quantities, the calculated best estimate predictions for our preferred standard solar models, BSB(GS98) and BSB(AGS05). The values that are not in parentheses were calculated using the Grevesse \& Sauval (1998) solar heavy element abundance (BSB(GS98) model); these values are very similar to those obtained with the solar model BS05(OP) of Bahcall et al. (2005c). The values that are in parentheses were calculated using the Asplund et al. (2005) recommended solar heavy element abundances (BSB(AGS05) model); these values correspond most closely to the values obtained from the solar model BS05(AGS, OP) of Bahcall et al. (2005c).

We now comment on some of the measurable quantities listed in Table 6. We first consider the predicted quantities that have been measured with helioseismology and then discuss briefly the quantities that have been measured by solar neutrino experiments.

\subsubsection{Measured Helioseismological Quantities}

For comparison with the value given in Table 6, the helioseismologically determined depth of the convective zone is (Kosovichev \& Fedorova 1991; Christensen-Dalsgaard et al. 1991; Guzik \& Cox 1993; Basu \& Antia 1997; Basu \& Antia 2004; Basu 1998)

$$
R_{\mathrm{CZ}}=0.713 \pm 0.001 R_{\odot} .
$$

The surface helium abundance of the Sun has recently been redetermined by Basu \& Antia (2004). They find

$$
Y_{\text {surf }}=0.2485 \pm 0.0034 \text {. }
$$

The interpretation of the errors given in equations (23) and (24) is not simple since systematic uncertainties are dominant. However, it is clear from Table 6 that the best estimates for $R_{\mathrm{CZ}}$ and
$Y_{\text {surface }}$ computed with the Grevesse \& Sauval (1998) abundances are in agreement with the measured values, while the best estimate values computed with the Asplund et al. (2005) differ noticeably from the measured values.

We will compare in $\S 6$ the Monte Carlo distributions for $R_{\mathrm{CZ}}$ and $Y_{\text {surface }}$ with the observed values given above. The profiles, $\delta c$ and $\delta \rho$, of the fractional differences, solar-model, of the sound speed and density are discussed in $\S 7$ and compared with helioseismological measurements. For completeness, we present in $\S 5.3$ the absolute values of the sound speed and density at different radii in the Sun in our standard models.

\subsubsection{Measured Solar Neutrino Quantities}

The measured event rate in the chlorine solar neutrino experiment, expressed in solar neutrino units (SNU), is (Cleveland et al. 1998)

$\left.\Sigma \phi(i) \sigma(i)\right|_{\mathrm{Cl}}=2.56 \pm 0.16$ (statistical) \pm 0.16 (systematic)SNU,

where the summation is over all eight of the neutrino fluxes shown in Table 6 . The difference between the predicted standard model value of the chlorine event rate and the measured event rate created the "solar neutrino problem" in 1968 (Bahcall et al. 1968; Davis et al. 1968). The predicted rates given in Table 6 for the $\mathrm{BSB}(\mathrm{GS} 98)$ and BSB(AGS05) solar models bracket the predicted value estimated in 1968 .

The neutrino absorption cross sections and their uncertainties used to calculate the predicted rate for the chlorine experiment shown in Table 6 are taken from Bahcall \& Ulrich (1988), except for the ${ }^{8} \mathrm{~B}$ absorption cross section, which is taken from Bahcall (1997). The uncertainties from the high-energy neutrinos (hep and ${ }^{8} \mathrm{~B}$ ) are calculated separately and combined quadratically with the uncertainties from the lower energy neutrinos (all other neutrino sources). The reason is that the lower energy neutrinos essentially cause only ground-state to ground-state nuclear transitions, whereas the hep and ${ }^{8} \mathrm{~B}$ neutrinos predominantly cause transitions to excited states. 
The weighted average rate measured by the SAGE, GALLEX, and GNO solar neutrino experiments is (Hampel et al. 1999; Abdurashitov et al. 2002, 2003; Altmann et al. 2005)

$$
\left.\Sigma \phi(i) \sigma(i)\right|_{\mathrm{Ga}}=68.1 \pm 3.85 \mathrm{SNU} .
$$

The neutrino absorption cross sections and their uncertainties used to calculate the predicted rate in the gallium experiments (see Table 6) are taken from Bahcall (1997). The uncertainties from the high-energy and low-energy neutrinos are combined quadratically, as explained above for the chlorine experiment.

The flux of electron neutrinos from ${ }^{8} \mathrm{~B}$ neutrino flux measured in the Kamiokande, Super-Kamiokande, and SNO experiments, assuming no distortion of the neutrino energy spectrum (no neutrino oscillations), is (Aharmim et al. 2005; Ahmed et al. 2004; Fukuda et al. 1996, 2001)

$$
\phi\left({ }^{8} \mathrm{~B}\right)_{e}=(1.68 \pm 0.10) \times 10^{6} \mathrm{~cm}^{-2} \mathrm{~s}^{-1} .
$$

The measured rates of electron type solar neutrinos determined in the chlorine, gallium, Kamiokande, Super-Kamiokande, and SNO experiments is, in all cases, much less than the rate predicted by the standard solar models. The discrepancies can be seen easily by comparing the values given in Table 6 with the values given in equations (25), (26), and (27).

The differences between the predicted standard model rates and the measured rates in the chlorine and gallium solar neutrino experiments are well explained by the hypothesis of solar neutrino oscillations (Gribov \& Pontecorvo 1969; Wolfenstein 1978; Mikheyev \& Smirnov 1985; Mikheyev \& Smirnov 1986; see, e.g., Bahcall et al. 2004a). The electron type neutrinos that are produced in the Sun and that have been measured directly on earth have mostly been converted to muon and tau neutrinos by the time they reach the terrestrial detectors. The quantitative disagreements between solar neutrino measurements and the predictions of the standard solar model, neglecting neutrino oscillations, are presented and discussed in $\S 8$.

By contrast, the total flux of ${ }^{8} \mathrm{~B}$ neutrinos (electron, muon, and tau neutrinos) determined by the SNO experiment (Aharmim et al. 2005, average of Phase I and Phase II measurements) is

$$
\phi\left({ }^{8} \mathrm{~B}\right)=(4.99 \pm 0.33) \times 10^{6} \mathrm{~cm}^{-2} \mathrm{~s}^{-1}
$$

which is in excellent agreement with the predicted ${ }^{8} \mathrm{~B}$ neutrino flux (see Table 6). In fact, the measured flux lies approximately halfway between the values predicted by the BSB(GS98) and the BSB(AGS05) solar models.

Given the reluctance to accept the solar model results by many physicists in the 1980s and 1990s (which led to the solar neutrino problem), it is of interest to compare the present best estimate rates for the standard solar model predictions with the values in the systematic study by Bahcall \& Ulrich (1988). Despite two decades of refinements in nuclear parameters, opacity, equation of state, and the inclusion of element diffusion, as well as intensive studies of the surface heavy element abundances, the neutrino predictions from the standard solar model remain almost unchanged. The 1988 prediction for the rate in the chlorine experiment (then the only available solar neutrino experiment) was 7.9 SNU (Bahcall \& Ulrich 1988), which is intermediate between the values of $8.1 \mathrm{SNU}$ and 6.6 SNU predicted, respectively, by the current BSB(GS98) and BSB(AGS05) solar models. The predicted gallium rate in 1988 was 132 SNU, which is 5\% (10\%) higher than the rate currently predicted with the BSB(GS98) and

\begin{tabular}{|c|c|}
\hline Quantity & Value \\
\hline \multicolumn{2}{|l|}{ Center } \\
\hline$T_{\mathrm{C}}$ & $15.67(15.48)$ \\
\hline$\rho_{\mathrm{C}}$ & $152.9(150.4)$ \\
\hline 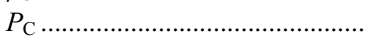 & $235.7(233.8)$ \\
\hline$X_{\mathrm{C}}$ & $0.3461(0.3647)$ \\
\hline$Y_{\mathrm{C}}$ & $0.6337(0.6202)$ \\
\hline \multicolumn{2}{|c|}{ Base of Convective Zone } \\
\hline$T_{\mathrm{CZ}} \ldots \ldots \ldots \ldots \ldots \ldots$ & $2.184(2.006)$ \\
\hline 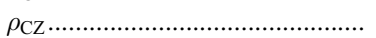 & $0.1862(0.1555)$ \\
\hline$P_{\mathrm{CZ}} \ldots \ldots \ldots \ldots \ldots \ldots \ldots \ldots \ldots \ldots \ldots \ldots \ldots \ldots \ldots \ldots \ldots \ldots \ldots \ldots \ldots \ldots \ldots$ & $0.05584(0.04341)$ \\
\hline 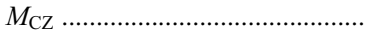 & $0.02403(0.01974)$ \\
\hline 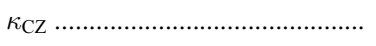 & $20.62(19.03)$ \\
\hline \multicolumn{2}{|c|}{ Other Quantities } \\
\hline$Y_{\text {init }}$ & $0.27250(0.26001)$ \\
\hline 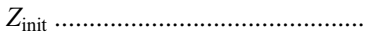 & $0.01884(0.01405)$ \\
\hline 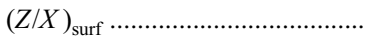 & $0.02292(0.01655)$ \\
\hline 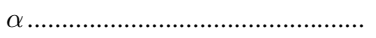 & $2.2097(2.1531)$ \\
\hline
\end{tabular}

TABLE 7

Some Characteristics of the Standard Solar Models

Notes.-Some characteristic solar model quantities. The table presents values calculated with the BSB(GS98) (no parentheses) standard solar model and the BSB(AGS05) standard model (in parentheses). Present-epoch central quantities are the temperature $T_{\mathrm{C}}$ (in units of $10^{6} \mathrm{~K}$ ), the density $\rho_{\mathrm{C}}$ (in units of $\mathrm{g} \mathrm{cm}^{-3}$ ), the pressure $P_{\mathrm{C}}$ in units of $\left(10^{15} \mathrm{erg} \mathrm{cm}^{-3}\right)$, as well as the hydrogen $X_{\mathrm{C}}$ and helium $Y_{\mathrm{C}}$ mass fractions. Conditions at the base of the convective zone are given by the temperature $T_{\mathrm{CZ}}$, density $\rho_{\mathrm{CZ}}$, pressure $P_{\mathrm{CZ}}$ (same units as before) and opacity $\kappa_{\mathrm{CZ}}$ (in units of $\mathrm{cm}^{2} \mathrm{~g}^{-1}$ ). $M_{\mathrm{CZ}}$ is the mass of the convective zone in units of solar masses. Finally, $Y_{\text {init }}, Z_{\text {init }},(Z / X)_{\text {surface }}$, and $\alpha$ are the initial helium mass fraction and metallicity, the present surface heavy metals to hydrogen mass fraction of the models, and the mixing length parameter.

$\mathrm{BSB}(\mathrm{AGS} 05)$ models. The best estimate value for the ${ }^{8} \mathrm{~B}$ neutrino flux was $5.76 \times 10^{6} \mathrm{~cm}^{-2} \mathrm{~s}^{-1}$, within $2 \%$ of the current prediction using the $\mathrm{BSB}(\mathrm{GS} 98)$ model. In all cases, the changes in the predictions for solar neutrino experiments have been less than the quoted theoretical errors given in 1988 (or now).

\subsection{Some Characteristics of the Standard Solar Models}

In this subsection, we present some characteristics of the standard solar model that are important and of general interest, but which - unlike the 23 quantities discussed in $\S 5.1$ - cannot be measured directly. Table 7 lists the central values of the temperature, density, and pressure, as well as the hydrogen mass fraction and the helium mass fraction. The values that are not enclosed in parentheses refer to the BSB(GS98) standard solar model, and the values in parentheses refer to the BSB(AGS05) solar model. The table also gives the values at the base of the convective zone of the temperature, density, and pressure, as well as the mass enclosed in the convective zone and the magnitude of the radiative opacity at the base of the zone. The last portion of the table gives the initial helium and heavy element abundance, the present-day surface abundance of $Z / X$, and the mixing length parameter.

At the present-epoch, the solar core in our standard models is contracting while the outer layers are expanding. The net effect is an increase in the gravitational binding energy of the Sun that releases energy at rate equal to $0.04 \%$ of the present solar luminosity, 
half of which is radiated away while the other half is stored as internal energy.

It is of interest to see how the characteristic parameters of the solar model have evolved over the last two decades, in which important refinements have been introduced into the calculations. The refinements include taking account of the diffusion of elements, using a more accurate radiative opacity and equation of state, and revising and refining the input nuclear cross sections. As a reference model, we use the Bahcall \& Ulrich (1988) standard solar model, which represented the first systematic combined investigation of the solar neutrino problem and of helioseismology and which was also the most comprehensive solar model study prior to the inclusion of element diffusion.

The central values of $T_{\mathrm{C}}, \rho_{\mathrm{C}}, P_{\mathrm{C}}, X_{\mathrm{C}}$, and $Y_{\mathrm{C}}$ for the Bahcall \& Ulrich (1988) model were 15.6, 148, 229, 0.3411, and 0.639 (same units as in the Table 7). We see by comparing the earlier values with the values given in Table 7 that the important improvements over the past two decades in the solar model physics have left the central parameters of the model almost unchanged.

On the other hand, the quantities at the base of the convective zone have changed considerably over the past two decades. The depth of the convective zone has moved deeper as the result of including element diffusion (Bahcall \& Pinsonneault 1995). In 1988 , the estimated depth of the convective zone was $0.74 R_{\odot}$, whereas the BSB(GS98) and the BSB(AGS05) solar models locate the base of the convective zone at 0.713 and $0.728 R_{\odot}$, respectively, in much better agreement with helioseismological measurements of the convective zone depth (see eq. [23]). All of the current best estimate parameters for the solar convective zone reflect the fact that the transition between radiative and convective energy transport occurs in a deeper part of the solar model than it did for the Bahcall \& Ulrich solar model.

In 1988, the best estimate for the initial helium abundance was $Y_{\text {init }}=0.271$, which is essentially identical to the current best estimated obtained with the BSB(GS98) solar model but is $4 \%$ larger than the best estimate obtained with the BSB(AGS05) model. The biggest change since 1988 is in the adopted ratio of $Z / X_{\text {surf }}$. In 1988, we used the value of $Z / X_{\text {surf }}=0.02765$ from Grevesse (1984), which is $21 \%$ larger than the Grevesse \& Sauval (1998) value and 67\% larger than the Asplund et al. (2005) ratio.

\subsection{Sound Speed and Density versus Radius}

In this subsection, we present and discuss the sound speed profile and the density profile in the Sun. These profiles are not directly measurable but are nevertheless of considerable theoretical interest. In $\S 7$, we compare the sound speed and density profiles in Monte Carlo solar models with the corresponding profiles in the Sun. Helioseismological inversions of solar observations determine not the absolute values of the sound speed and density that are discussed in the present subsection, but rather the differences between the model and solar profiles that are discussed in $\S 7$.

Table 8 presents the sound speeds as a function of solar radius for both the BSB(GS98) and the BSB(AGS05) standard solar models. The sound speed in the solar models varies from about $500 \mathrm{~km} \mathrm{~s}^{-1}$ in the solar center to about $8 \mathrm{~km} \mathrm{~s}^{-1}$ on the solar surface. The two standard solar models have very similar sound speed profiles. The relative difference of the solar sound speed between the BSB(GS98) and BSB(AGS05) models in the convective envelope is about $-0.05 \%$. At the base of the convective zone the sound speed of BSB(GS98) becomes larger and the relative difference has a maximum of about $1 \%$ at $0.65 R_{\odot}$. From that point inward, the difference decreases, becoming negative again at $0.3 R_{\odot}$ and reaches the value of $-0.04 \%$ at the solar center.
TABLE 8

The Sound Speed in the Sun as a Function of Radius for the Standard Solar Models BSB(GS98) and BSB(AGS05)

\begin{tabular}{|c|c|c|}
\hline$R / R_{\odot}$ & $\mathrm{BSB}(\mathrm{GS} 98)$ & $\mathrm{BSB}(\mathrm{AGS} 05)$ \\
\hline $0.000 \ldots \ldots$ & $5.0666 \mathrm{E}+02$ & $5.0873 \mathrm{E}+02$ \\
\hline 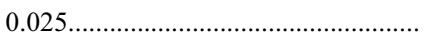 & $5.0803 \mathrm{E}+02$ & $5.0996 \mathrm{E}+02$ \\
\hline 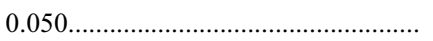 & $5.1074 \mathrm{E}+02$ & $5.1236 \mathrm{E}+02$ \\
\hline $0.075 \ldots \ldots$ & $5.1167 \mathrm{E}+02$ & $5.1304 \mathrm{E}+02$ \\
\hline $0.100 \ldots \ldots \ldots$ & $5.0838 \mathrm{E}+02$ & $5.0963 \mathrm{E}+02$ \\
\hline 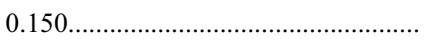 & $4.8748 \mathrm{E}+02$ & $4.8852 \mathrm{E}+02$ \\
\hline $0.200 \ldots \ldots \ldots \ldots$ & $4.5498 \mathrm{E}+02$ & $4.5569 \mathrm{E}+02$ \\
\hline 0.250 & $4.2068 \mathrm{E}+02$ & $4.2100 \mathrm{E}+02$ \\
\hline 0.300 & $3.8941 \mathrm{E}+02$ & $3.8933 \mathrm{E}+02$ \\
\hline 0.350 & $3.6228 \mathrm{E}+02$ & $3.6178 \mathrm{E}+02$ \\
\hline 0.400 & $3.3866 \mathrm{E}+02$ & $3.3773 \mathrm{E}+02$ \\
\hline $0.450 \ldots \ldots$ & $3.1782 \mathrm{E}+02$ & $3.1647 \mathrm{E}+02$ \\
\hline 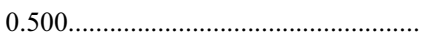 & $2.9912 \mathrm{E}+02$ & $2.9737 \mathrm{E}+02$ \\
\hline $0.550 \ldots \ldots \ldots \ldots$ & $2.8188 \mathrm{E}+02$ & $2.7976 \mathrm{E}+02$ \\
\hline $0.600 \ldots \ldots$ & $2.6531 \mathrm{E}+02$ & $2.6295 \mathrm{E}+02$ \\
\hline $0.650 \ldots \ldots$ & $2.4831 \mathrm{E}+02$ & $2.4599 \mathrm{E}+02$ \\
\hline $0.675 \ldots \ldots$ & $2.3923 \mathrm{E}+02$ & $2.3707 \mathrm{E}+02$ \\
\hline $0.700 \ldots \ldots \ldots \ldots . . . .$. & $2.2940 \mathrm{E}+02$ & $2.2767 \mathrm{E}+02$ \\
\hline 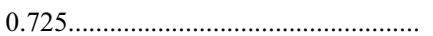 & $2.1702 \mathrm{E}+02$ & $2.1699 \mathrm{E}+02$ \\
\hline 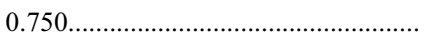 & $2.0345 \mathrm{E}+02$ & $2.0358 \mathrm{E}+02$ \\
\hline 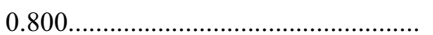 & $1.7603 \mathrm{E}+02$ & $1.7613 \mathrm{E}+02$ \\
\hline $0.850 \ldots \ldots \ldots$ & $1.4748 \mathrm{E}+02$ & $1.4755 \mathrm{E}+02$ \\
\hline $0.900 \ldots \ldots \ldots \ldots$ & $1.1609 \mathrm{E}+02$ & $1.1614 \mathrm{E}+02$ \\
\hline 0.920 & $1.0201 \mathrm{E}+02$ & $1.0207 \mathrm{E}+02$ \\
\hline $0.930 \ldots \ldots \ldots-1$ & $9.4440 \mathrm{E}+01$ & $9.4501 \mathrm{E}+01$ \\
\hline $0.940 \ldots \ldots \ldots-1$ & $8.6375 \mathrm{E}+01$ & $8.6441 \mathrm{E}+01$ \\
\hline 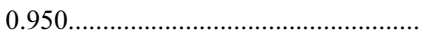 & $7.7661 \mathrm{E}+01$ & $7.7731 \mathrm{E}+01$ \\
\hline $0.960 \ldots \ldots \ldots \ldots \ldots \ldots$ & $6.8017 \mathrm{E}+01$ & $6.8093 \mathrm{E}+01$ \\
\hline $0.970 \ldots \ldots \ldots \ldots \ldots \ldots$ & $5.6939 \mathrm{E}+01$ & $5.7020 \mathrm{E}+01$ \\
\hline $0.980 \ldots \ldots \ldots \ldots \ldots \ldots$ & $4.4299 \mathrm{E}+01$ & $4.4401 \mathrm{E}+01$ \\
\hline 0.990 & $2.8017 \mathrm{E}+01$ & $2.7985 \mathrm{E}+01$ \\
\hline 1.000 & $7.9193 \mathrm{E}+00$ & $7.9889 \mathrm{E}+00$ \\
\hline
\end{tabular}

Notes.-The tabulated values of $c$ are the sound speed in $\mathrm{km} \mathrm{s}^{-1}$. More extensive numerical tables of $c$ are available at http://www.sns.ias.edu/ jnb.

Quadratic interpolation within Table 8 accurately reproduces the numerical values from the solar models. The sound speed can be interpolated from the table to an accuracy that is typically much better than $0.1 \%$ from the center of the Sun up to $0.98 R_{\odot}$. Only in the region $0.15 R_{\odot}<R<0.2 R_{\odot}$ is the accuracy of the quadratic interpolation degraded to about $0.15 \%$.

Table 9 presents the density profiles for the BSB(GS98) and $\mathrm{BSB}(\mathrm{AGS} 05)$ standard solar models. The density varies by 9 orders of magnitude from the solar interior to the solar surface, from $153 \mathrm{~g} \mathrm{~cm}^{-3}\left(150 \mathrm{~g} \mathrm{~cm}^{-3}\right)$ at the center of the Sun to $1.65 \times 10^{-7} \mathrm{~g} \mathrm{~cm}^{-3}\left(1.68 \times 10^{-7} \mathrm{~g} \mathrm{~cm}^{-3}\right)$ at the solar surface (optical depth equal 0.312). Quadratic interpolation in Table 9 reproduces the solar model densities to an accuracy better than $0.2 \%$ in the inner $0.8 R_{\odot}$ and is more accurate than $0.5 \%$ up to $0.97 R_{\odot}$.

The fractional differences between the densities for the $\mathrm{BSB}(\mathrm{GS} 98)$ and the BSB(AGS05) solar models are larger than the fractional differences of the sound speeds. The density in the convective envelope is about 7\% larger in the BSB(GS98) model than in the BSB(AGS05) model. This difference smoothly drops to zero at $0.45 R_{\odot}$, where it becomes negative and at $0.25 R_{\odot}$ the $\mathrm{BSB}(\mathrm{AGS} 05)$ density is about $1 \%$ larger than that of the BSB(GS98) model. Toward the center the BSB(GS98) model again has higher density than the BSB(AGS05) model, the difference being close to $2 \%$ in the center. 
TABLE 9

The Logarithm of the Total Density in the Sun as a Function of Radius for the Standard Solar Models BSB(GS98) and BSB(AGS05)

\begin{tabular}{|c|c|c|}
\hline$R / R_{\odot}$ & BSB(GS98) & BSB(AGS05) \\
\hline $0.000 \ldots \ldots \ldots \ldots \ldots \ldots$ & 2.185 & 2.177 \\
\hline 0.025 & 2.164 & 2.158 \\
\hline 0.050 & 2.109 & 2.104 \\
\hline 0.075 & 2.032 & 2.028 \\
\hline 0.100 & 1.943 & 1.941 \\
\hline 0.150 & 1.752 & 1.753 \\
\hline $0.200 \ldots \ldots \ldots$ & 1.546 & 1.548 \\
\hline $0.250 \ldots \ldots$ & 1.321 & 1.325 \\
\hline $0.300 \ldots \ldots \ldots$ & 1.082 & 1.085 \\
\hline $0.350 \ldots \ldots$ & 0.836 & 0.839 \\
\hline $0.400 \ldots$ & 0.592 & 0.593 \\
\hline $0.450 \ldots \ldots \ldots \ldots \ldots$ & 0.355 & 0.354 \\
\hline 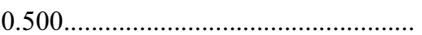 & 0.127 & 0.124 \\
\hline 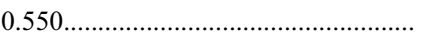 & -0.091 & -0.097 \\
\hline $0.600 \ldots \ldots$ & -0.299 & -0.309 \\
\hline $0.650 \ldots \ldots$ & -0.496 & -0.512 \\
\hline 0.675 & -0.591 & -0.610 \\
\hline $0.700 \ldots \ldots$ & -0.684 & -0.706 \\
\hline $0.725 \ldots \ldots \ldots$ & -0.768 & -0.798 \\
\hline $0.750 \ldots \ldots$ & -0.853 & -0.883 \\
\hline $0.800 \ldots \ldots$ & -1.042 & -1.073 \\
\hline 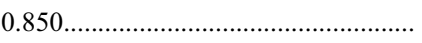 & -1.274 & -1.304 \\
\hline $0.900 \ldots \ldots$ & -1.586 & -1.616 \\
\hline 0.920 & -1.754 & -1.784 \\
\hline 0.930 & -1.854 & -1.884 \\
\hline 0.940 & -1.970 & -2.000 \\
\hline 0.950 & -2.108 & -2.138 \\
\hline 0.960 & -2.280 & -2.309 \\
\hline $0.970 \ldots \ldots \ldots$ & -2.508 & -2.537 \\
\hline $0.980 \ldots \ldots \ldots$ & -2.852 & -2.880 \\
\hline $0.990 \ldots \ldots \ldots$ & -3.506 & -3.533 \\
\hline 1.000 & -6.783 & -6.774 \\
\hline
\end{tabular}

Notes.-The tabulated values are $\log _{10} \rho$, where $\rho$ is the total density in $\mathrm{g} \mathrm{cm}^{-3}$. More extensive numerical tables of $\rho$ and are available at http://www.sns.ias.edu/ $\sim$ jnb.

\subsection{Neutrino Fluxes versus Radius}

Figure 1 shows the production profiles versus radius of each of the important solar neutrino fluxes, as well as the solar luminosity. The ${ }^{8} \mathrm{~B},{ }^{7} \mathrm{Be},{ }^{15} \mathrm{O}$, and ${ }^{17} \mathrm{~F}$ neutrino fluxes are concentrated toward the center of the Sun, while the $p-p$, pep, and hep fluxes are more broadly distributed.

The ${ }^{13} \mathrm{~N}$ neutrino production profile is double-peaked. The inner peak (small radii) represents neutrinos produced where the $\mathrm{CN}$ reactions are approximately in steady state. The outer peak (large radii) represents the residual burning of ${ }^{12} \mathrm{C}$ by the reaction ${ }^{12} \mathrm{C}(p, \gamma){ }^{13} \mathrm{~N}\left(\beta^{+}\right){ }^{13} \mathrm{C}$ at radii at which the temperature is too low to permit the subsequent burning of nitrogen.

Table 10 gives the locations of the peak in the flux distribution per unit radius for each solar neutrino flux, as well as the locations below and above the peak radius within which $34.1 \%$ (effective $\sigma / 2$ ) of the flux is produced. The table presents values that were computed using the Grevesse \& Sauval (1998) recommended heavy element abundances and also values that were computed using the Asplund et al. (2005) recommended abundances. One can see immediately from the table that the flux distributions are practically independent of which of the two recommended compositions is used, which is another indication that solar neutrino fluxes are not much affected by the choice of heavy element composition (within the currently fashionable range of surface heavy element abundances).
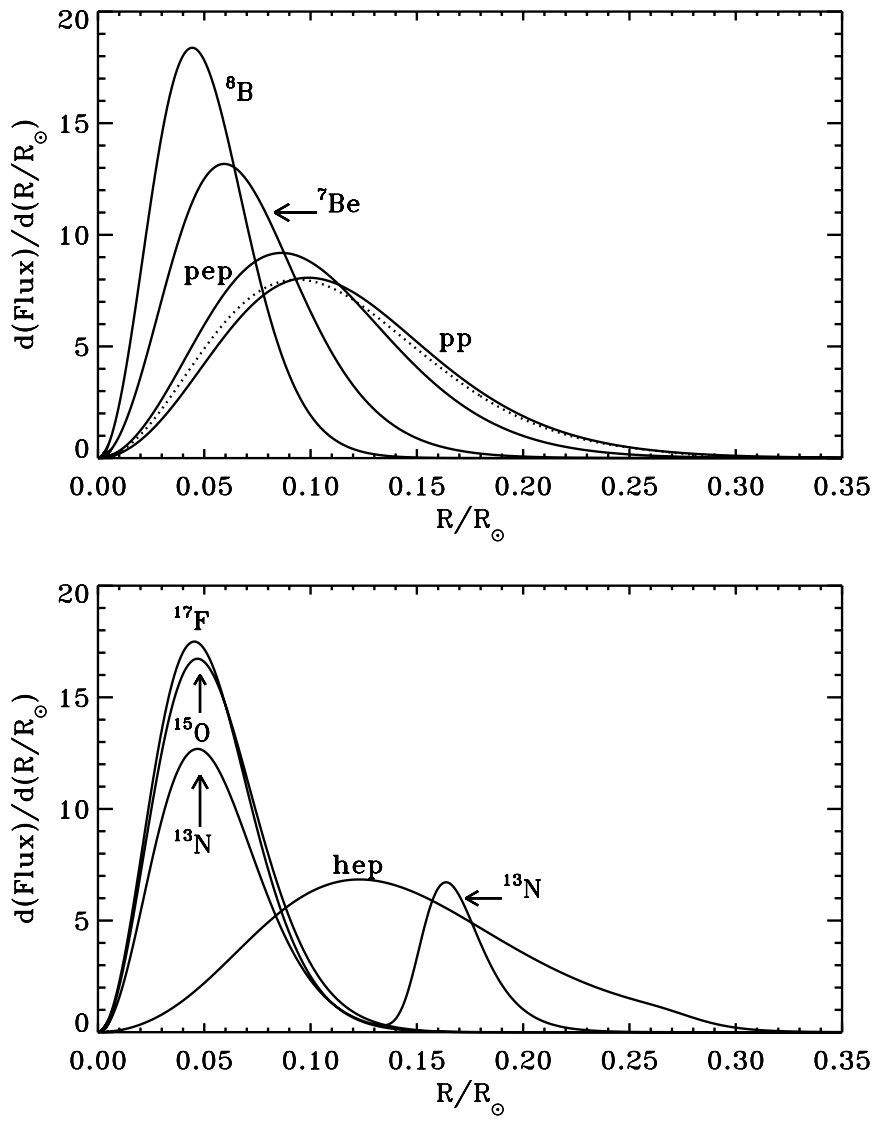

FIg. 1.-Neutrinos fluxes vs. radius. The figure shows the production profiles of the principal neutrino fluxes vs. radius for our standard solar model $\mathrm{BSB}(\mathrm{GS} 98)$. The dotted line in the upper panel, close to the profile for the $p-p$ neutrino flux, represents the production profile of the solar luminosity. The production profiles are normalized to unity when integrated over $d R / R_{\odot}$. The double-peaked distribution of the ${ }^{13} \mathrm{~N}$ neutrino flux is explained in the text.

TABLE 10

Neutrino Flux Dependence on Radius

\begin{tabular}{|c|c|c|c|c|c|c|}
\hline \multirow[b]{2}{*}{$\begin{array}{l}\text { Neutrino Flux } \\
\text { (1) }\end{array}$} & \multicolumn{3}{|c|}{ BSB(GS98) } & \multicolumn{3}{|c|}{ BSB(AGS05) } \\
\hline & $\begin{array}{l}R_{\text {peak }} \\
\text { (2) }\end{array}$ & $\begin{array}{l}R_{\text {inner }} \\
\text { (3) }\end{array}$ & $\begin{array}{l}R_{\text {outer }} \\
\text { (4) }\end{array}$ & $\begin{array}{c}R_{\text {peak }} \\
(5)\end{array}$ & $\begin{array}{c}R_{\text {inner }} \\
\text { (6) }\end{array}$ & $\begin{array}{l}R_{\text {outer }} \\
\text { (7) }\end{array}$ \\
\hline & 0.0990 & 0.0470 & 0.1471 & 0.0990 & 0.0471 & 0.1472 \\
\hline pep....... & 0.0864 & 0.0410 & 0.1286 & 0.0866 & 0.0411 & 0.1290 \\
\hline hep ....... & 0.1230 & 0.0616 & 0.1796 & 0.1230 & 0.0618 & 0.1797 \\
\hline${ }^{7} \mathrm{Be} .$. & 0.0594 & 0.0276 & 0.0889 & 0.0592 & 0.0274 & 0.0887 \\
\hline${ }^{8} \mathrm{~B} \ldots$ & 0.0443 & 0.0220 & 0.0654 & 0.0442 & 0.0219 & 0.0653 \\
\hline${ }^{13} \mathrm{~N} \ldots .$. & 0.0468 & 0.0221 & 0.0698 & 0.0470 & 0.0224 & 0.0701 \\
\hline${ }^{13} \mathrm{~N}_{2} \ldots \ldots \ldots \ldots \ldots \ldots \ldots \ldots$ & 0.1637 & 0.1473 & 0.1781 & 0.1615 & 0.1450 & 0.1758 \\
\hline $15 \mathrm{O}$ & 0.0468 & 0.0220 & 0.0700 & 0.0470 & 0.0222 & 0.0703 \\
\hline${ }^{17} \mathrm{~F}$ & 0.0454 & 0.0217 & 0.0675 & 0.0455 & 0.0218 & 0.0677 \\
\hline
\end{tabular}

Notes.-The table presents the peak radius, $R_{\text {peak }}$, and the inner and outer radii, $R_{\text {inner }}$ and $R_{\text {outer }}$, for each neutrino flux. The peak radius corresponds to the maximum of the flux production per unit radius, while the inner and outer radii correspond to the points at which $34.1 \%$ of the flux is produced on either side of the peak. The flux distributions are shown in Fig. 1. The second ${ }^{13} \mathrm{~N}$ peak, ${ }^{13} \mathrm{~N}_{2}$, can be seen clearly in Fig. 1 and is explained in the text. The second, third, and fourth columns were computed using GS98 heavy element abundances; the fifth, sixth, and seventh columns were computed using AGS05 heavy element abundances. 
TABLE 11

The Electron Number Density and the Neutron Number Density vs. Radius for the Standard Solar Models (GS98 and AGS05, see $\S 5.1$ )

\begin{tabular}{|c|c|c|c|c|}
\hline \multirow[b]{2}{*}{$R / R_{\odot}$} & \multicolumn{2}{|c|}{$\log _{10}\left(n_{e} / N_{\mathrm{A}}\right)$} & \multicolumn{2}{|c|}{$\log _{10}\left(n_{n} / N_{\mathrm{A}}\right)$} \\
\hline & GS98 & AGS05 & GS98 & AGS05 \\
\hline $0.000 \ldots \ldots \ldots \ldots \ldots \ldots \ldots$ & 2.0125 & 2.0114 & 1.6990 & 1.6795 \\
\hline 0.025 & 1.9981 & 1.9974 & 1.6665 & 1.6476 \\
\hline 0.050 & 1.9581 & 1.9585 & 1.5770 & 1.5593 \\
\hline 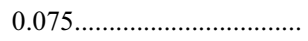 & 1.8998 & 1.9015 & 1.4495 & 1.4325 \\
\hline 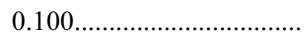 & 1.8295 & 1.8326 & 1.3037 & 1.2862 \\
\hline 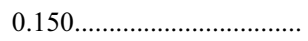 & 1.6648 & 1.6703 & 1.0102 & 0.9901 \\
\hline $0.200 \ldots$ & 1.4711 & 1.4783 & 0.7440 & 0.7223 \\
\hline $0.250 \ldots$ & 1.2509 & 1.2591 & 0.4950 & 0.4729 \\
\hline $0.300 \ldots \ldots$ & 1.0129 & 1.0211 & 0.2462 & 0.2236 \\
\hline $0.350 \ldots \ldots \ldots \ldots$ & 0.7678 & 0.7752 & -0.0050 & -0.0285 \\
\hline 0.400 & 0.5244 & 0.5303 & -0.2519 & -0.2769 \\
\hline 0.450 & 0.2877 & 0.2916 & -0.4911 & -0.5183 \\
\hline 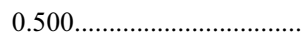 & 0.0602 & 0.0614 & -0.7209 & -0.7509 \\
\hline 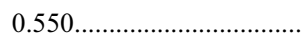 & -0.1574 & -0.1594 & -0.9407 & -0.9740 \\
\hline $0.600 \ldots \ldots \ldots \ldots$ & -0.3649 & -0.3708 & -1.1501 & -1.1878 \\
\hline 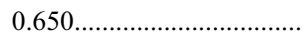 & -0.5621 & -0.5732 & -1.3489 & -1.3919 \\
\hline 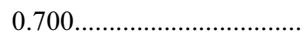 & -0.7460 & -0.7651 & -1.5582 & -1.6008 \\
\hline $0.750 \ldots \ldots \ldots \ldots$ & -0.9130 & -0.9394 & -1.7395 & -1.8012 \\
\hline $0.800 \ldots \ldots \ldots$ & -1.1024 & -1.1287 & -1.9289 & -1.9906 \\
\hline 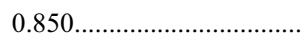 & -1.3341 & -1.3603 & -2.1605 & -2.2221 \\
\hline 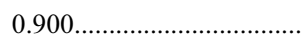 & -1.6462 & -1.6721 & -2.4726 & -2.5340 \\
\hline $0.940 \ldots \ldots \ldots \ldots \ldots \ldots \ldots$ & -2.0306 & -2.0561 & -2.8571 & -2.9180 \\
\hline 0.950 & -2.1685 & -2.1938 & -2.9950 & -3.0557 \\
\hline $0.960 \ldots$ & -2.3399 & -2.3648 & -3.1663 & -3.2267 \\
\hline $0.970 \ldots \ldots \ldots$ & -2.5683 & -2.5926 & -3.3947 & -3.4545 \\
\hline 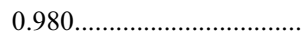 & -2.9124 & -2.9355 & -3.7388 & -3.7973 \\
\hline $0.990 \ldots \ldots \ldots \ldots \ldots \ldots . .$. & -3.5662 & -3.5890 & -4.3927 & -4.4508 \\
\hline 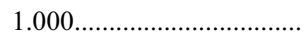 & -6.8436 & -6.8296 & -7.6700 & -7.6915 \\
\hline
\end{tabular}

Notes.-The tabulated values are $\log _{10}\left(n_{e} / N_{\mathrm{A}}\right)$ and $\log _{10}\left(n_{n} / N_{\mathrm{A}}\right)$, where $n_{e}\left(n_{n}\right)$ is the electron (neutron) number density measured in number per $\mathrm{cm}^{3}$ and $N_{\mathrm{A}}$ is Avogadro's number. More extensive numerical files of $n_{e}$ and $n_{n}$ are available at http://www.sns.ias.edu/ jnb. The numerical values were calculated with our preferred solar models constructed using either GS98 or AGS05 heavy element abundances and are described in $\S 5$ and Table 6.

\subsection{Electron and Neutron Number Densities versus Radius}

We present in this subsection the electron and neutron number densities that are required to calculate the propagation of neutrinos through solar material. The dominant effect for converting an electron type neutrino to a muon or tau neutrino in the Sun is proportional to the profile of the electron number density minus one-half the neutron number density, $n_{e}-0.5 n_{n}$, as a function of solar radius (Wolfenstein 1978; Mikheyev \& Smirnov 1985; Lim \& Marciano 1988). This matter-induced change of neutrino flavors is known as the MSW effect, after its discoverers Mikheyev, Smirnov, and Wolfenstein. The probability for matter to induce transformations of other active neutrinos, $\nu_{\mu}$ or $\nu_{\tau}$, is proportional to $-0.5 n_{n}$. If one only considers active neutrinos, $\nu_{e}, \nu_{\mu}$, and $\nu_{\tau}$, then it is not necessary to know $n_{n}$ because the common phase induced by $n_{n}$ does not affect the oscillation probability. However, in order to calculate the propagation of sterile neutrinos, one requires the profile of $n_{e}-0.5 n_{n}$ (Barger et al. 1991).

We present here the separate distributions for $n_{e}$ and $n_{n}$. The user can easily form the combined density $n_{e}-0.5 n_{n}$ from the values given here. In addition, since we give separately $n_{e}$ and $n_{n}$ the user can study more exotic, nonstandard interactions that may require different combinations of $n_{e}$ and $n_{n}$ (see, e.g., Wolfenstein 1978; Friedland et al. 2004).

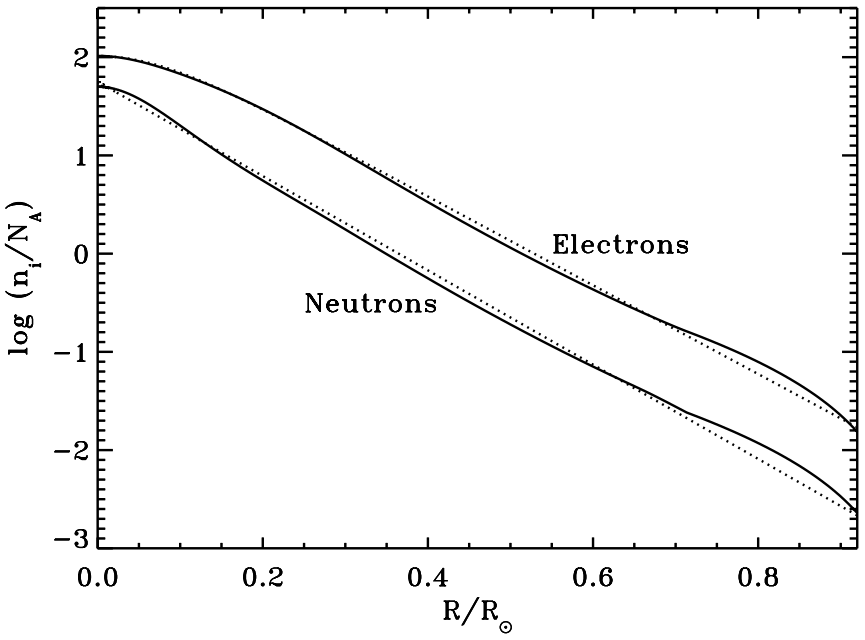

FIG. 2.-Electron and neutron number densities vs. radius. The figure shows as solid lines the logarithm of the number densities of electrons and of neutrons, divided by Avogadro's number $N_{\mathrm{A}}$, vs. radius. The dotted lines represent the analytic fits presented in the text, eqs. (29) and (30). For radii $R<0.3 R_{\odot}$, the analytic fits are sufficiently close to the actual model distributions that it is difficult to see them as separate lines in the figure. The electron and neutron number densities plotted in this figure were calculated using a solar model that incorporated GS98 heavy element abundances. The number densities are practically the same if AGS05 heavy element abundances are used, see text and Table 11.

Table 11 gives, at representative points in the Sun, the electron and neutron number densities as a function of solar radius for our standard solar model. For the electron distribution, the values given in Table 11 can be used (with quadratic interpolation) to reproduce the actual electron distribution in the given solar models to better than $0.1 \%$ in all the solar interior from the center up to $0.7 R_{\odot}$ and, for larger radii, to better than $2 \%$ up to $0.99 R_{\odot}$. For the neutron distribution, quadratic interpolation in Table 11 reproduces the solar models distribution to an accuracy of $0.5 \%$ (usually much better) from the center up to $0.7 R$. and, for larger radii, to an accuracy of $2 \%$ up to $0.99 R_{\odot}$. More extensive numerical files of the number densities are available online (see footnote 2 ).

As can be seen in Table 11, the electron and neutron density distributions are different for different assumed solar compositions. In the case of the electron density distribution, the differences between the models are smaller than $2 \%$ for $R<0.6 R_{\odot}$ and rise up to $6 \%$ in the convective envelope $\left(R>0.7 R_{\odot}\right)$, where the higher values correspond to the BSB(GS98) model. On the other hand, the neutron density is $5 \%$ higher for the $\mathrm{BSB}(\mathrm{GS} 98$ ) model at the center and this difference smoothly increases up to $10 \%$ at the base of the convective envelope, while in the convective envelope itself the difference is about $15 \%$.

Figure 2 shows the calculated solar model values of the electron and neutron number densities versus solar radius for the standard solar model constructed with the GS98 heavy element abundances. In the inner region of the Sun, $R<0.3 R_{\odot}$, where matter effects are most relevant for neutrino oscillations, the electron and neutron number densities can be approximated by analytic formulae. We find for the electron number density

$\log n_{e} / N_{\mathrm{A}}=2.36-4.52 x-0.33 \exp \left[(-x / 0.075)^{1.1}\right]$

and for the neutron number density

$$
\log n_{n} / N_{\mathrm{A}}=1.72-4.80 x,
$$


where $x=R / R_{\odot}$. The analytic fits given in equations (29) and (30) are shown as dotted lines in Figure 2.

The first two terms in equation (29) (with slightly different constants), originally derived by Bahcall \& Ulrich (1988; see Bahcall 1989), have been used by many authors in calculations of MSW survival probabilities. However, these two terms alone significantly overestimate the electron number density for radii less than $0.12 R_{\odot}$ (see Fig. $6 d$ of Bahcall \& Ulrich 1988 or Fig. $4 d$ of Bahcall 1989). We have therefore added the third term in equation (29), which leads to satisfactory agreement with the numerical values for the solar model in the inner region of the Sun. The rms difference between the values given by equation (29) is 7\% for $R<0.7 R_{\odot}$. The agreement of the values given in equation (30) with the solar model values is $12 \% \mathrm{rms}$ for $R<$ $0.7 R_{\odot}$. In the outer region, for $R>0.8 R_{\odot}$ (which is less important for standard MSW transformations), the analytic fits represented by equations (29) and (30) are not accurate and one must use the more precise numerical values extrapolated from Table 11 or take the values directly from the solar model.

For our standard solar model constructed with AGS05 heavy element abundances, the coefficients for the corresponding versions of equations (29) and (30) are practically the same. For the three coefficients in the AGS05 version of equation (29) the numerical constants are, respectively, 2.38, 4.56, and -0.36 (instead of the values of $2.36,4.52$, and -0.33 that are optimal for GS98 abundances). For the AGS05 version of equation (30), the numerical constants are 1.72 and -4.84 (instead of 1.72 and -4.80 for GS98 abundances).

The analytic formulae given in equations (29) and (30) can be used in analytic discussions of solar neutrino oscillations (just as they have been used by many authors in previous decades), and for most purposes these formulae are adequate for numerical calculations of neutrino oscillations. However, for the most precise work, quadratic interpolation in Table 11 should be used.

\section{MONTE CARLO RESULTS FOR CONVECTIVE ZONE DEPTH AND SURFACE HELIUM ABUNDANCE}

We present in this section our Monte Carlo results for the depth of the solar convective zone and the surface helium abundance. We compare the results with the helioseismologically measured values. We begin by discussing in $\S 6.1$ the comparison between the calculated and measured values for the depth of the convective zone. We then discuss in $\S 6.2$ the comparison between the calculated and measured values of the surface helium abundance.

Our major results are summarized in Figure 3. All of the panels in the figure show the number of solar models that were found to have the depth of the convective zone (or, for the righthand panels, the surface helium abundance) in a given bin. The top two rows of the figure were obtained from 5000 solar models each, and the bottom (lowest) row summarizes the results for 1000 solar models. All of the distributions are well fitted (as judged by a reduced $\chi^{2}$ calculation) by a Gaussian shape, which is shown in each panel as a smooth curve.

\subsection{Depth of The Convective Zone}

The top two left panels of Figure 3 summarize the results for the depth of the convective zone that were obtained with our two standard composition choices: (1) GS98 abundances and "conservative" uncertainties (GS98-Cons); (2) AGS05 abundances and "optimistic" uncertainties (AGS05-Opt). The third (lowest) row was computed with a hybrid choice of AGS05 abundances and conservative uncertainties (AGS05-Cons).

The GS98 abundances and the "conservative" composition uncertainties listed in the second row of Table 3 were used in calculating the solar models to which the top panel refers. The mean value and $1 \sigma$ uncertainty for the calculated values of the convective zone are

$$
R_{\mathrm{CZ}}=0.7154 \pm 0.0102 R_{\odot} ; \quad \text { GS98-Cons. }
$$

In this case, the solar model results are in good agreement with the helioseismologically determined depth of the convective zone (see eq. [23]).

However, the situation is different if the AGS05 abundances and the AGS05 uncertainties ("optimistic" uncertainties, see col. [3] of Table 3) are both used. The second row of Figure 3 shows that the disagreement in this case is significant. We find

$$
R_{\mathrm{CZ}}=0.7280 \pm 0.0037 R_{\odot} ; \quad \text { AGS05-Opt. }
$$

Thus, the composition and composition uncertainties recommended by Asplund et al. (2005) lead to solar models with values for $R_{\mathrm{CZ}}$ that differ from the helioseismologically measured value by $3.7 \sigma$, where the $\sigma$ used here is the quadratically combined solar model and helioseismological errors.

If the AGS05 (Asplund et al. 2005) abundances and the "conservative" composition uncertainties are used to calculate the depth of the convective zone, we find (see second row of Fig. 3)

$$
R_{\mathrm{CZ}}=0.7296 \pm 0.0105 R_{\odot} ; \quad \text { AGS05-Cons. }
$$

There is no strong disagreement between the AGS05 abundances and the measured depth of the convective zone if conservative composition uncertainties are assumed. Note, however, that this a result of the large conservative composition uncertainties.

\subsection{Surface Helium Abundance}

The right-hand panels of Figure 3 compare the solar model calculations of the present-day surface helium abundance with the helioseismologically measured values. Using the GS98 abundances and conservative uncertainties, we find (see Fig. 3, top right)

$$
Y_{\text {surf }}=0.2420 \pm 0.0072 ; \quad \text { GS98-Cons },
$$

which is in very good agreement with the helioseismologically determined value of $Y_{\text {surf }}=0.2485$ (see eq. [24]).

The AGS05 abundances and uncertainties lead to solar model predictions,

$$
Y_{\text {surf }}=0.2292 \pm 0.0037 ; \quad \text { AGS05-Opt }
$$

that differ from the helioseismologically measured value by $3.8 \sigma$ (combined solar model and helioseismological error).

The agreement is still not good if we use AGS05 abundance and conservative uncertainties. In this case, we find from the solar models that

$$
Y_{\text {surf }}=0.2285 \pm 0.0067 ; \quad \text { AGS05-Cons. }
$$

The best-estimate with the AGS05 abundances and conservative uncertainties differs from the helioseismologically measured value (eq. [24]) by about $2.7 \sigma$.

\section{MONTE CARLO RESULTS FOR SOUND SPEED AND DENSITY PROFILES}

In the previous section we examined the distribution of the position of the $\mathrm{CZ}$ base and the helium abundance of the models. 

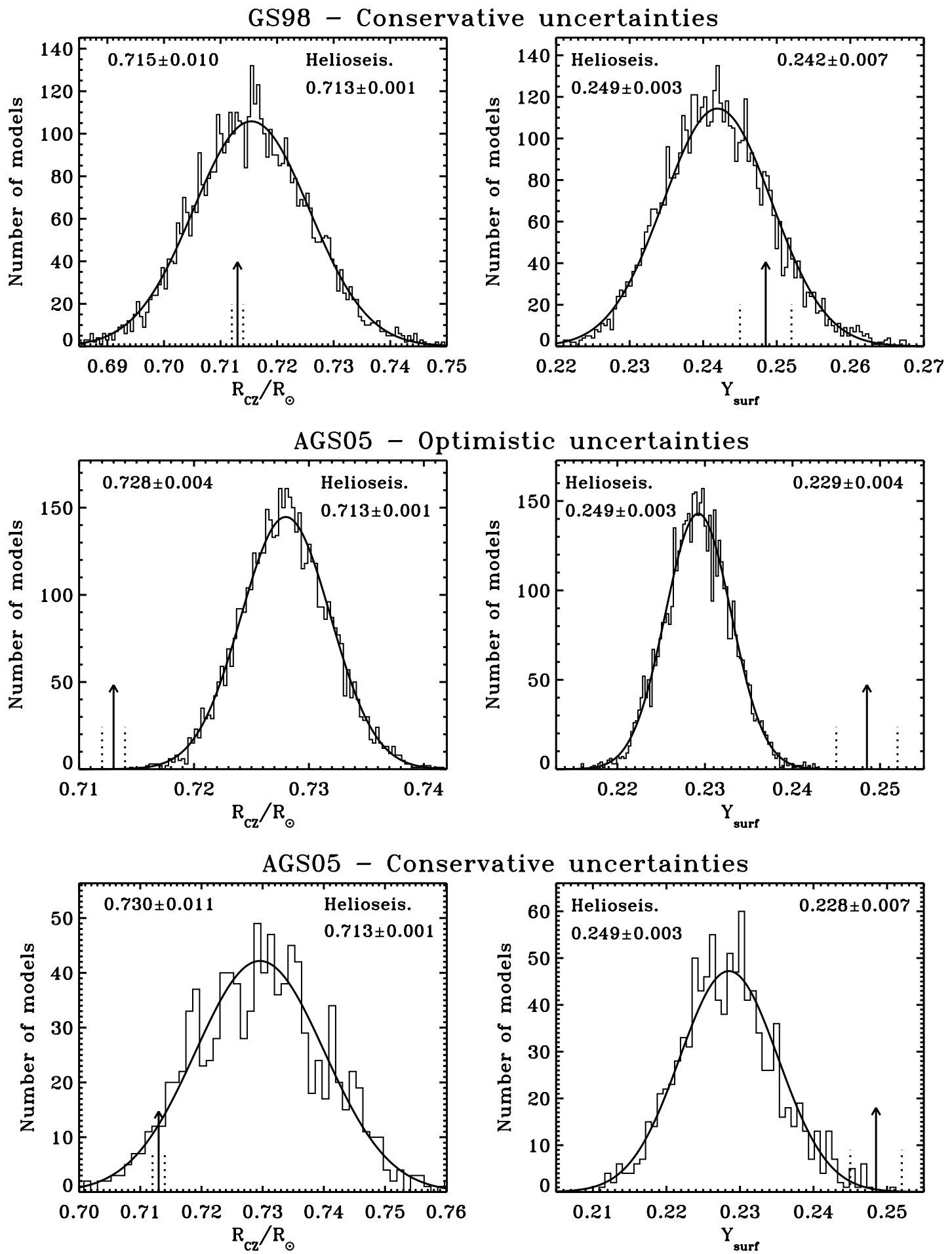

FIg. 3.-Depth of the convective zone, $R_{\mathrm{cz}}$ and surface helium abundance, $Y_{\text {surf }}$. The three left panels present the number of models with different values of the depth of the convective zone for (top) conservative composition errors and Grevesse \& Sauval (1998) recommended abundances; (middle) the recommended errors and abundances given by Asplund et al. (2005); and (bottom) conservative composition errors and the Asplund et al. (2005) recommended abundances. The composition uncertainties are discussed in $\$ 2.2$. The right three panels show the number of solar models with different values of the surface helium abundance for the same three cases as for the depth of the convective zone. The arrows and the dotted lines represent the measured values and their uncertainties. The distribution of models is, in all six cases shown in Fig. 3 , well described with a Gaussian distribution, the smooth curves in each panel. The confidence level in the top two plots is, e.g., $96.3 \%$ and $80.0 \%$.

While the CZ base and helium abundance are two very important quantities obtained from helioseismic studies of the Sun, they are by no means the only two. Helioseismic inversions have allowed us to determine the solar sound-speed and density profiles for most of the Sun, the results are valid from roughly $5 \%-7 \%$ of the solar radius to $95 \%$ of the solar radius. Thus, we have additional information about the Sun with which to compare our models. We present in this section the Monte Carlo results for the comparison between the solar model and helioseismologically obtained solar sound-speed and density profiles.

Inversions to determine solar the solar sound-speed and density profiles are done by inverting the frequency differences 
between the Sun and a solar model to obtain the sound-speed and density between the Sun and the model (called the "reference model"). Thus, inversions directly show us whether or not the structure of a solar model agrees with that of the Sun. For this work, the frequency differences between the Sun and the models were inverted using the Subtractive Optimally Localized Averages (SOLA) technique (Pijpers \& Thompson 1992; Pijpers \& Thompson 1994). We set up the inversion problem and determined the various parameters of the inversion in the manner described by Rabello-Soares et al. (1999). For the helioseismological data, we use solar oscillation frequencies obtained by the Michelson Doppler Imager (MDI) on board the Solar and Heliospheric Observatory $(\mathrm{SOHO})$. In particular, we use frequencies obtained from MDI data that were collected for the first 360 days of its observation (Schou et al. 1998). This data set was chosen because it was derived from a long time series when solar activity was low. The length of the time series results in reduced noise and hence a larger number of modes for which the frequencies can be determined reliably. Mode sets derived from longer data sets are available, but they only consist of low-degree modes (e.g., Bertello et al. 2000). In addition, a longer time series would have meant adding observations from periods of increasing solar activity, which would have changed the frequencies. It is a well-established fact that solar frequencies increase with solar activity. However, it is also known that increase is such that it does not reflect a change in structure of the solar interior (Basu 2002). To quantize the difference between the structure of the Sun and the models, we determine the rms difference between the sound-speed and density profiles of the Sun and the models. A larger rms difference would denote a worse model. We note here that in order to minimize systematic effects, the inversion of the solar frequencies has been done independently with each of the models in our simulation as the reference model.

We present the results discussion the sound-speed differences between the Sun and the different models in $\S 7.1$. The density differences are described in $\S 7.2$. The results are summarized by Figures 4 and 5, as well as Table 12 .

\subsection{Sound Speed Profiles}

The rms difference between the sound-speed profile of the Sun and the models is shown in Figure 4. The differences are shown not only for the entire range of radii over which the inversion results are valid, but also a few smaller radius ranges to check for the sensitivity of the profiles to input physics.

The internal structure of solar models is sensitive, at different depths, to different physical inputs in the calculations. For instance, the adopted solar composition has a more dramatic effect on the structure of the models for $R \gtrsim 0.45 R_{\odot}$ (Bahcall et al. $2005 \mathrm{a}, 2005 \mathrm{~b})$. Of particular importance is how composition affects the location of the base of the solar convective envelope and how this affects the sound-speed profile in the region $R \gtrsim$ $0.45 R_{\odot}$. In the temperature range in this region, opacity is dominated by bound-free transitions, and this largely determines the temperature gradient. Higher metal abundances lead to higher opacities and a steeper radiative temperature gradient. As a result, the condition for convective stability (for which we adopt the Schwarzschild criterion) is satisfied at higher temperatures (i.e., a larger depth) for a higher metallicity. Given the sharp transition in temperature at the $\mathrm{CZ}$ base, a mismatch in its location in the solar models with respect to the actual solar location gives rise to a large difference sound speed in that region. At inner regions, however, free-free transitions and electron scattering opacity sources become gradually more important, diminishing the influence of the details of the solar composition. This leads us to discuss our results for the sound speed profiles (and analogously for the density profiles) not only in terms of the rms sound speed difference $\delta c_{\text {all }}$ over the entire range of validity of the inversion, but also in two smaller ranges, an inner rms $\delta c_{\text {inner }}$ defined for $0.07 R_{\odot} \leq R \leq 0.45 R_{\odot}$, and an outer sound speed difference rms $\delta c_{\text {outer }}$ defined for $0.45 R_{\odot} \leq R \leq 0.95 R_{\odot}$ (see also $\$ 3.1)$.

The resulting distributions for $\delta c_{\text {all }}, \delta c_{\text {inner }}$, and $\delta c_{\text {outer }}$ are shown in Figure 4 (top, middle, and bottom panels, respectively). The column on the left corresponds to the models obtained from the Monte Carlo simulations adopting the GS98-Cons composition choice, while the right column corresponds to models obtained with the AGS05-Opt composition. In the case of the GS98-Cons simulation, the distribution of each of the difference rms is strongly peaked very close to zero, reinforcing the well-known result that solar models adopting the GS98 solar abundances give in general very good agreement with helioseismic results regardless of the uncertainties in the other input physics (e.g., nuclear cross section, EOS, radiative opacities, element diffusion). The tails extending to high rms values result from the adopted large conservative uncertainties. For the AGS05Opt composition simulation the distributions peak at much higher values than in the GS98-Cons case, reflecting the fact that the sound speed of the solar models constructed with this composition are not in good agreement with the sound speed profile inferred from helioseismology. In addition, it is evident that the distributions do not extend to such low rms values as those found with the GS98 composition.

Table 12 summarizes our results by quantitatively characterizing the distributions we have obtained in our Monte Carlo simulations for the rms differences. In the top three rows of Table 12 we characterize the three sound speed difference rms distributions for both the GS98-Cons and AGS05-Opt simulations and, for completeness, the hybrid AGS05-Cons case. In the case of the GS98-Cons composition we characterize each of the rms distributions by giving their most probable value (mode) that we denote by $Q$, and their one-sided $68.3 \%(1 \sigma)$ confidence level value, $\sigma_{0.68}$ (for each quantity of interest, $\sigma_{0.68}$ is defined such that $68.3 \%$ of all the models in the Monte Carlo simulation have this quantity in the interval $\left.\left[0, \sigma_{0.68}\right]\right)$. For the AGS05-Opt case, we find that the distributions are well described by lognormal distributions, and in Table 12 we give their mode $Q$ and the $1 \sigma$ confidence level interval $\left[M-\sigma_{-}, M+\sigma_{+}\right]$(see Appendix for details on how $\sigma_{-}$and $\sigma_{+}$are defined). Solar models using the AGS05 composition show a worse agreement with helioseismology results than models using the GS98 composition. This is evident from Figure 4. An indicative measure of the degradation is given by the ratio of the most probable values of the $\delta c_{\text {all }}$ distributions for each composition choice, e.g., $Q_{\delta c \text {,all }}(\mathrm{AGS} 05) / Q_{\delta c \text {,all }}(\mathrm{GS} 98)$, and we find that this number is $0.00487 / 0.00143=3.4$.

From Figure 4 and Table 12 it is evident that the largest contribution to $\delta c_{\text {all }}$ originates in the outer half of the solar model. Note in particular the smaller scale of the abscissa of the middle panels as compared to the top and bottom panels. This shows that the sound-speed difference between the Sun and the models is quite low in the region that includes the core. Indeed, the low sound-speed difference in the core is the basis of the helioseismic solution of the solar neutrino problem (e.g., Bahcall et al. 1997). The larger values of $\delta c_{\text {outer }}$ can be understood by noting that the region over which the quantity is defined includes, in addition to the convective envelope, the radiative layers immediately below it. The change in temperature gradient at the base of the convection zone causes a large change in sound-speed, and a mismatch of the $\mathrm{CZ}$ base position between the models and 

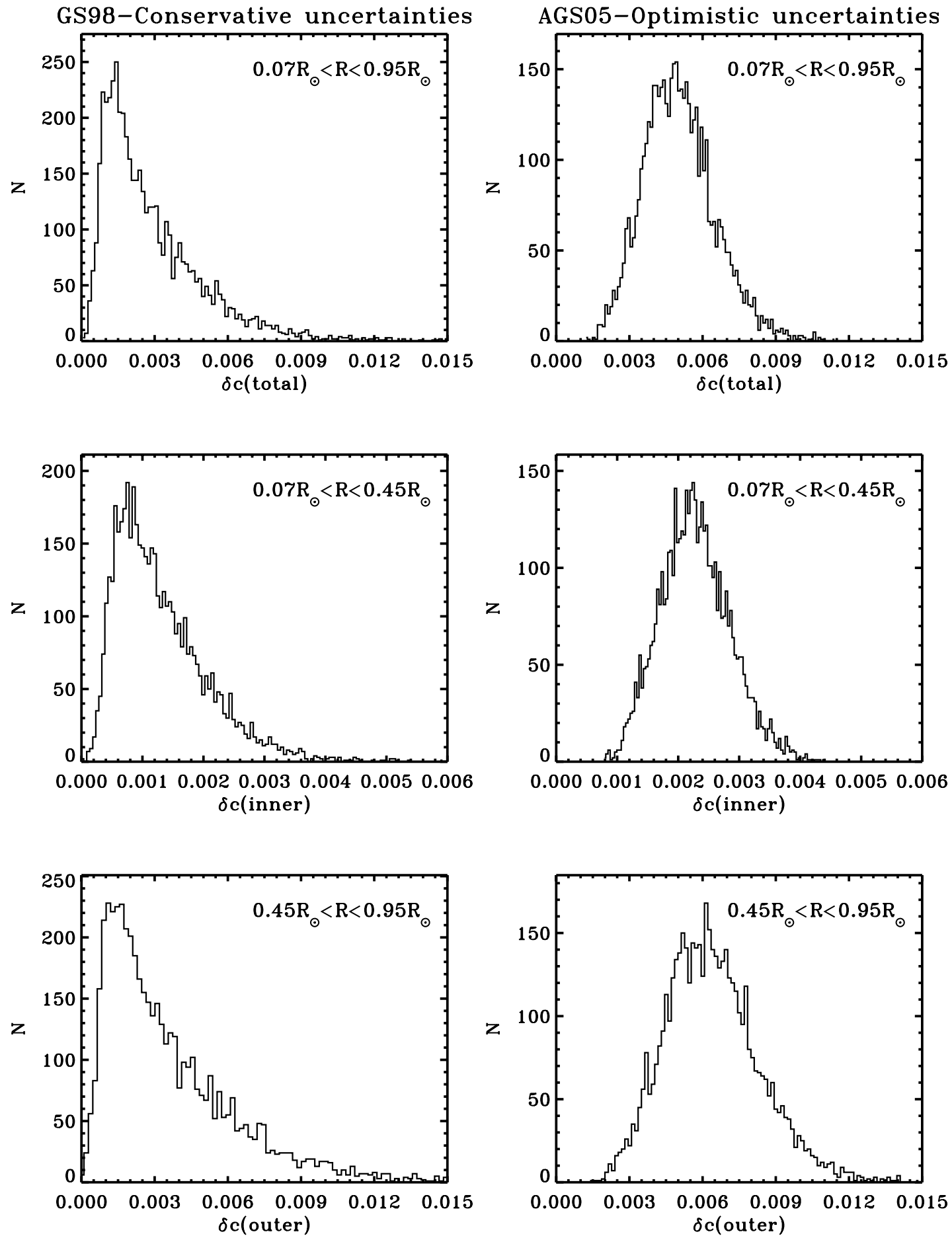

FIG. 4.-Distributions of the rms sound-speed differences. Panels on the left (right) correspond to the GS98-Cons (AGS05-Opt) composition choice. From top to bottom, the total $\delta c_{\text {all }}$, inner $\delta c_{\text {inner }}$, and outer $\delta c_{\text {outer }}$ rms differences are shown. The solar region involved in the definition of each rms difference is shown in the respective panels.

the Sun translates to relatively large differences in the inferred sound speed profiles. In the case of the GS98-Cons composition, for instance, the ratio of the most probable values of the $\delta c_{\text {outer }}$ and $\delta c_{\text {inner }}$ distributions is $Q_{\delta c \text {,outer }} / Q_{\delta c \text {,inner }}=1.5$. The effect of a wrong location of $R_{\mathrm{CZ}}$ is more evident in the simulations adopting the AGS05-Opt composition, for which the difference between the predicted $R_{\mathrm{CZ}}$ and that measured by helioseismology becomes very large. In this case we get $Q_{\delta c \text {,outer }} / Q_{\delta c \text {,inner }}=2.7$. This reflects the fact that $\delta c_{\text {outer }}$ is more affected than $\delta c_{\text {inner }}$ by the adoption the AGS05 solar composition, and the underlying reason is the effect of composition on opacities.

\subsection{Density Profiles}

We present the results for the differences in the density profiles in a manner similar to those of the sound-speed differences. The results are shown in Figure 5. As in the case of sound speed, we define the rms density difference between the Sun and the models $-\rho_{\text {all }}, \rho_{\text {inner }}$, and $\rho_{\text {outer }}$ - with definitions analogous to the sound-speed difference case. The last three rows of Table 12 show the characteristics of the distributions of $\rho_{\text {all }}, \rho_{\text {inner }}$, and $\rho_{\text {outer }}$ that we find for our model obtained from the Monte Carlo simulations. 

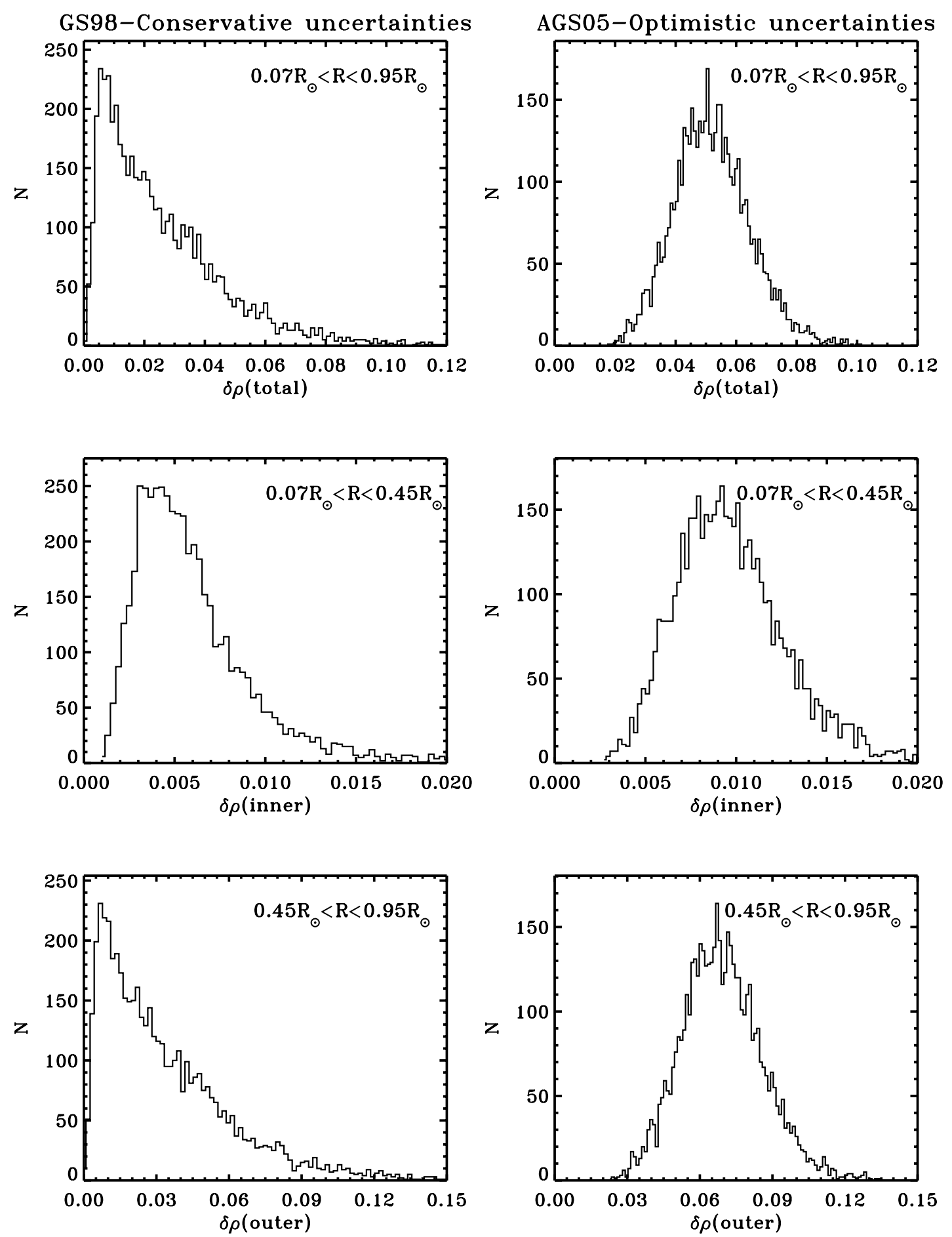

FIG. 5.-Density rms difference distributions. Panels on the left (right) correspond to the GS98-Cons (AGS05-Opt) composition choice. From top to bottom, the total $\delta \rho_{\text {all }}$, inner $\delta \rho_{\text {inner }}$, and outer $\delta \rho_{\text {outer }}$ rms differences are shown. The solar region involved in the definition of each rms difference is shown in the respective panels.

Figure 5 presents, from top to bottom, the distributions for $\rho_{\text {all }}, \rho_{\text {inner }}$, and $\rho_{\text {outer }}$ and for the GS98-Cons (left column) and AGS05-Opt (right column) composition choices. Again, distributions from the GS98-Cons simulation are one-sided distributions. Although this may not be strictly true in the case of the $\rho_{\text {inner }}$ distribution, it is nevertheless a highly asymmetric distribution and we keep this description for the sake of simplicity. AGS05-Opt results are again well described by lognormal distributions. Again, the hybrid AGS05-Cons results are included in the table for the sake of completeness.
Qualitatively, results for the rms density differences resemble those for the sound-speed differences, although the density differences are generally larger than the sound-speed differences. As in the case of sound speed, the distributions for the GS98Cons composition are strongly peaked close to zero, showing the consistency between standard solar models that adopt this composition and the helioseismological inferences for the solar density profile. On the other hand, the Monte Carlo simulation with the AGS05-Opt composition give distributions for $\rho_{\text {all }}$, $\rho_{\text {inner}}$, and $\rho_{\text {outer }}$ that show a much degraded agreement with 
TABLE 12

Sound Speed and Density Profiles: Root Mean Square Differences between Solar Models and Helioseismological Measurements

\begin{tabular}{|c|c|c|c|c|c|c|c|c|}
\hline \multirow[b]{2}{*}{$\begin{array}{l}\text { DIFFERENCE } \\
\text { (1) }\end{array}$} & \multicolumn{2}{|c|}{ GS98-Cons } & \multicolumn{3}{|c|}{ AGS05-Opt } & \multicolumn{3}{|c|}{ AGS05-Cons } \\
\hline & $\begin{array}{c}Q \\
(2)\end{array}$ & $\begin{array}{c}\sigma_{0.68} \\
(3)\end{array}$ & $\begin{array}{c}Q \\
(4)\end{array}$ & $\begin{array}{c}\sigma_{+} \\
(5)\end{array}$ & $\begin{array}{l}\sigma_{-} \\
(6)\end{array}$ & $\begin{array}{c}Q \\
(7)\end{array}$ & $\begin{array}{c}\sigma_{+} \\
(8)\end{array}$ & $\begin{array}{l}\sigma_{-} \\
(9)\end{array}$ \\
\hline 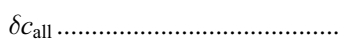 & 0.00143 & 0.00334 & 0.00487 & 0.00136 & 0.00119 & 0.00484 & 0.00316 & 0.00256 \\
\hline 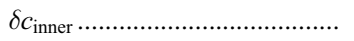 & 0.00075 & 0.00151 & 0.00216 & 0.00060 & 0.00052 & 0.00238 & 0.00096 & 0.00095 \\
\hline$\delta c_{\text {outer }}$ & 0.00111 & 0.00423 & 0.00582 & 0.00206 & 0.00167 & 0.00523 & 0.00453 & 0.00312 \\
\hline 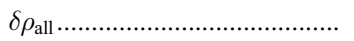 & 0.0055 & 0.0311 & 0.0486 & 0.0135 & 0.0112 & 0.0440 & 0.0311 & 0.0224 \\
\hline$\delta \rho_{\text {inner }}$ & 0.0039 & 0.0067 & 0.0086 & 0.0032 & 0.0025 & 0.0076 & 0.0057 & 0.0033 \\
\hline$\delta \rho_{\text {outer }} \ldots \ldots \ldots \ldots \ldots \ldots \ldots \ldots \ldots \ldots$ & 0.0069 & 0.0408 & 0.0646 & 0.0180 & 0.0149 & 0.0580 & 0.0416 & 0.0295 \\
\hline
\end{tabular}

Notes.-The rms differences $\delta c$ and $\delta \rho$ of the sound speed and the density profiles from the helioseismologically determined profiles are given in the table for three regions of the solar interior. Eqs. (17) and (18) define the rms differences. In the case of the GS98 abundances and conservative uncertainties the distributions are highly asymmetric and we characterize them by their most probable value (or mode) $Q$ (col. [2]) and their one-sided $68.3 \%$ confidence level $\sigma_{0.68}$ (col. [3]). For the AGS05 composition and optimistic uncertainties we give for the distribution of each rms difference the mode $Q$ (col. [4]) and the $\sigma_{+}$ and $\sigma_{-}$values defining the $68.3 \%$ confidence level (cols. [5] and [6], respectively). The same quantities are given for the AGS05 composition and conservative uncertainties in cols. (7)-(9). Details on the definitions of $\sigma_{0.68}, \sigma_{+}$, and $\sigma_{-}$are given in the text. The three regions are (inner) $0.07 R_{\odot} \leq R \leq$ $0.45 R_{\odot}$; (outer) $0.45 R_{\odot} \leq R \leq 0.95 R_{\odot}$; and (all) $0.07 R_{\odot} \leq R \leq 0.95 R_{\odot}$.

helioseismology. An indicative value of this degradation is given by the ratio of the most probable values $Q_{\delta \rho, \text { all }}(\mathrm{AGS05}) /$ $Q_{\delta \rho, \text { all }}(\mathrm{GS} 98)=0.0486 / 0.0055 \approx 8.8$. This seems to indicate that, despite the fact that solar density profile is somewhat less well determined by inversions of the solar frequencies than the solar sound speed profile, the density profile in the solar models is very sensitive to the input physics adopted. Besides, it is known that envelope models for the Sun can be constructed with near perfect sound speed differences even when the AGS05 composition is adopted (Basu \& Antia 2004; Antia \& Basu 2005), these models, however, still have a density profile in disagreement with that determined from helioseismology. This points in the direction that, although somewhat more limited from the observational point of view, density profiles can be a powerful helioseismological tool. This appears to be particularly true in the case of the problem posed by the new determinations of the solar composition.

From the results in this and the previous subsection, we conclude that the disagreement between the standard solar model predictions and helioseismological measurements of the solar sound-speed and density profiles introduced by the adoption of the new solar composition (Asplund et al. 2005), is unlikely to be explained by changing the other input physics included in standard solar models within the currently accepted uncertainties. This result strengthens those described in $\S 6$, where we compared the helium abundance and depth of the convective zone of the models with that of the Sun.

\section{MONTE CARLO RESULTS FOR INDIVIDUAL NEUTRINO FLUXES}

We present in this section the Monte Carlo results for the distribution of each of the neutrino fluxes and their total uncertainties from all sources for the predicted solar neutrino fluxes. Here results are presented, as in other sections, for the three separate cases, which are distinguished by which heavy element composition and by which set of composition uncertainties are adopted, as explained in $\S \S 1.1$ and 2.2.

In terms of the resulting shape of the distributions, we find that the neutrino fluxes can be separated into two groups, regardless of the composition choice. The first group is formed by the $p-p$, pep, hep, and ${ }^{7} \mathrm{Be}$ neutrino fluxes. For these fluxes, we find by $\chi^{2}$ analysis that their distributions can be described as normal distributions to better than a $95 \%$ confidence level. The only exception is the ${ }^{7} \mathrm{Be}$ flux distribution for the GS98-Cons composition choice, for which the confidence level is $70 \%$. The second group is formed by the fluxes that have uncertainties dominated by the composition uncertainties, i.e., the ${ }^{8} \mathrm{~B},{ }^{13} \mathrm{~N}$, ${ }^{15} \mathrm{O}$, and ${ }^{17} \mathrm{~F}$ neutrino fluxes. The distributions of these fluxes are very well described by lognormal distributions, the $\chi^{2}$ analyses yield confidence levels better than $97 \%$ in all cases, which reflect our assumptions regarding the solar composition uncertainties.

For the four most experimentally important neutrino fluxes, the $p$ - $p$, pep, ${ }^{7} \mathrm{Be}$, and ${ }^{8} \mathrm{~B}$ neutrino fluxes, we present histograms for all three composition options. In Figures 6 and 7, the top, middle, and bottom rows correspond to the GS98-Cons, AGS05Opt, and AGS05-Cons composition choices, respectively. For the more difficult to measure hep, ${ }^{13} \mathrm{~N},{ }^{15} \mathrm{O}$, and ${ }^{17} \mathrm{~F}$ neutrinos, we present histograms only for the GS98-Cons case.

Tables 13 and 14 give the parameters needed to characterize the fluxes distributions of our simulations. Table 15 summarizes the Monte Carlo uncertainties for all eight neutrino fluxes and for all three assumptions regarding the composition.

Our Monte Carlo technique only provides direct results for the total uncertainties of each neutrino flux. However, dominant contributions from individual sources to the total uncertainty can generally be identified with the aid of the input standard deviations given in Tables 3, 4, and 5. In the following subsections, we comment on the dominant individual sources of uncertainty for each flux where this seems relevant.

Section 8.1 describes the histogram of results for the ${ }^{8} \mathrm{~B}$ neutrinos that have been measured directly in the Kamiokande (Fukuda et al. 1996), Super-Kamiokande (Fukuda et al. 2001, 2002), and SNO experiments (Ahmed et al. 2004; Aharmim et al. 2005). We then discuss in $\S 8.2$ the ${ }^{7} \mathrm{Be}$ neutrinos that will be studied in the Borexino experiment (Alimonti et al. 2002) and perhaps the KamLAND experiment (Araki et al. 2005) or the LENA experiment (Oberauer et al. 2005). Section 8.3 presents results for the calculated Monte Carlo distribution of the fundamental $p-p$ and pep neutrino fluxes. This section also provides predictions for the anticorrelations of the $p-p$ and $p e p$ neutrino fluxes with the ${ }^{7}$ Be neutrino flux (see Fig. 8), as well as the predicted correlation between the $p-p$ and $p e p$ fluxes (see Fig. 9). We present in $\S 8.4$ the results for the difficult-to-measure hep $,{ }^{13} \mathrm{~N},{ }^{15} \mathrm{O}$, and ${ }^{17} \mathrm{~F}$ neutrino fluxes.

\subsection{The ${ }^{8}$ B Neutrino Flux}

The three panels on the left side of Figure 6 show the histograms of the number of computed models with ${ }^{8} \mathrm{~B}$ neutrino fluxes in each flux bin. The assumed abundances and 

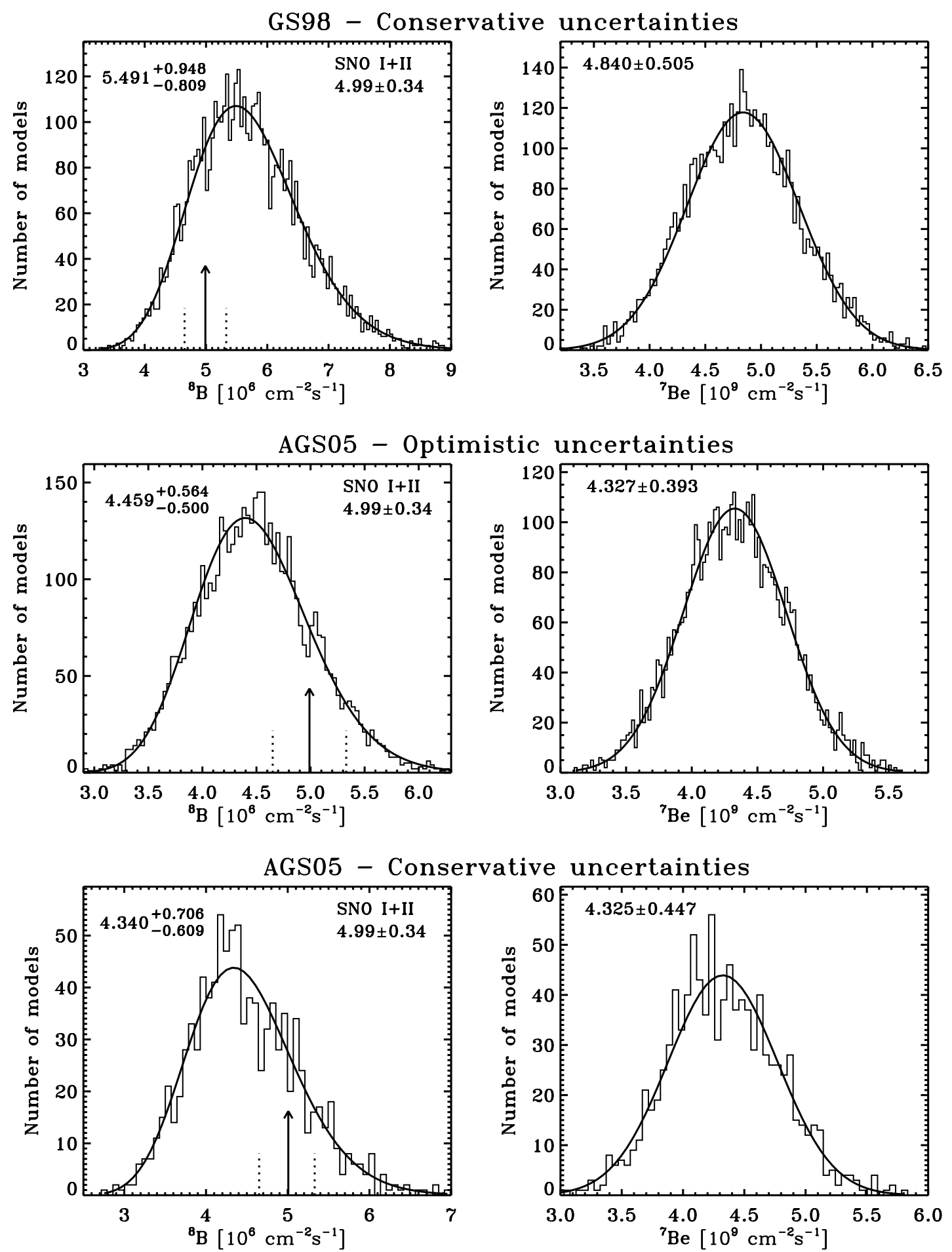

Fig. 6.-The ${ }^{8} \mathrm{~B}$ and ${ }^{7} \mathrm{Be}$ neutrino fluxes. The figure shows the number of solar models from our Monte Carlo simulations that have ${ }^{8} \mathrm{~B}$ (left panels) and ${ }^{7} \mathrm{Be}($ right panels) neutrino fluxes in the indicated ranges. From top to bottom, rows refer to the GS98-Cons, AGS05-Opt, and AGS05-Cons composition choices, respectively. The conservative and optimistic abundance uncertainties are given in Table 3. The smooth curves represent the lognormal (normal) distributions inferred for the ${ }^{8} \mathrm{~B}\left({ }^{7} \mathrm{Be}\right)$ flux distributions from our simulations. For the ${ }^{8} \mathrm{~B}$ flux the mode $Q$ and $\sigma_{+}$and $\sigma_{-}$as defined in the text are given in each panel. In the case of the ${ }^{7} \mathrm{Be}$ flux, the mean and the standard deviation are given. Fluxes units are the same as in Table 6.

abundance uncertainties are written above each of the three rows of panels.

The weighted average value of the SNO neutral current measurements from the Neutral Current Phase I and Phase II measurements (Ahmed et al. 2004; Aharmim et al. 2005) is (4.99士 $0.34) \times 10^{6} \mathrm{~cm}^{-2} \mathrm{~s}^{-1}$. This value is shown as an arrow perpendicular to the horizontal axis of each of the ${ }^{8} \mathrm{~B}$ panels, together with a dotted line that shows the $1 \sigma$ uncertainty of the weighted average. The figure shows that adopting either of the recommended set of heavy element abundances, GS98 or AGS05, leads to good agreement with the total ${ }^{8} \mathrm{~B}$ neutrino flux measured by the SNO neutral current experiments (see BP04; Bahcall et al. $2005 \mathrm{c}$ ). The measured value of the ${ }^{8} \mathrm{~B}$ neutrino flux falls slightly below the best-fit solar model prediction if GS98 abundances are 

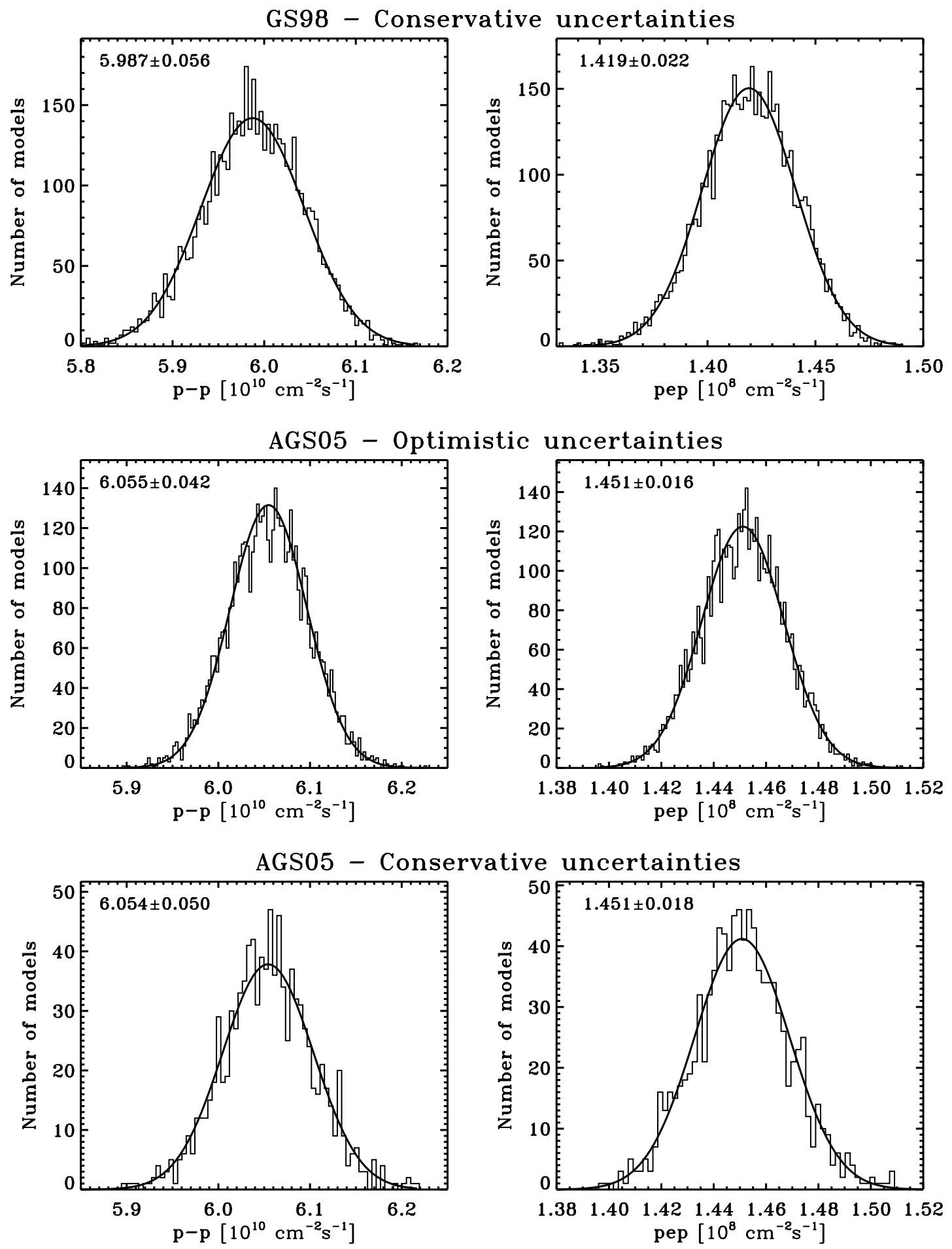

FIG. 7.- The $p$ - $p$ and pep neutrino fluxes. This figure is the same as Fig. 6 except that the present figure refers to $p-p$ and $p e p$ solar neutrinos rather than ${ }^{8} \mathrm{~B}$ and ${ }^{7} \mathrm{~B}$

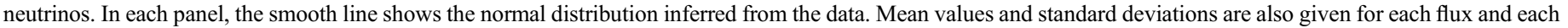
composition case.

adopted (Fig. 6, upper left) and is slightly higher than the best-fit value if AGS05 abundances are assumed. The ${ }^{8} \mathrm{~B}$ solar neutrino flux is not very sensitive to which of the two heavy element compositions, GS98 or AGS05, is incorporated into the solar models.

The effect of the composition uncertainties is, however, noticeable in the shape of the ${ }^{8} \mathrm{~B}$ flux distribution. It is apparent, particularly in the top and bottom left panels of Figure 6, that the ${ }^{8} \mathrm{~B}$ flux distributions are not symmetric. This is a consequence of the assumed distribution for the composition uncertainties
(§ 2.3). As anticipated, we find that the ${ }^{8} \mathrm{~B}$ flux distribution of our Monte Carlo simulations can be very well described by lognormal distributions (with confidence levels better than 98.5\%) for all composition choices. The parameters characterizing each of these distributions are given in Table 14. A summary of the properties of lognormal distributions relevant to this paper is given in the Appendix.

Table 15 and Figure 6 show that the total $1 \sigma$ theoretical uncertainty in the predicted ${ }^{8} \mathrm{~B}$ neutrino flux varies from $17 \%$ to 
TABLE 13

Neutrino FluXes with Gaussian Distributions

\begin{tabular}{|c|c|c|c|c|c|c|}
\hline \multirow[b]{2}{*}{$\begin{array}{l}\text { FLUX } \\
\text { (1) }\end{array}$} & \multicolumn{2}{|c|}{ GS98-Cons } & \multicolumn{2}{|c|}{ AGS05-Орт } & \multicolumn{2}{|c|}{ AGS05-Cons } \\
\hline & $\begin{array}{c}\mu \\
(2)\end{array}$ & $\begin{array}{c}\sigma \\
(3)\end{array}$ & $\begin{array}{c}\mu \\
(4)\end{array}$ & $\begin{array}{c}\sigma \\
(5)\end{array}$ & $\begin{array}{c}\mu \\
(6)\end{array}$ & $\begin{array}{c}\sigma \\
(7)\end{array}$ \\
\hline$p-p \ldots \ldots$ & 5.987 & 0.056 & 6.055 & 0.042 & 6.054 & 0.050 \\
\hline 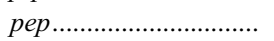 & 1.419 & 0.022 & 1.451 & 0.016 & 1.451 & 0.018 \\
\hline hep & 7.970 & 1.236 & 8.251 & 1.276 & 8.281 & 1.264 \\
\hline${ }^{7} \mathrm{Be}$ & 4.840 & 0.505 & 4.327 & 0.393 & 4.325 & 0.447 \\
\hline
\end{tabular}

Notes.-Parameters of the Gaussian distributions that describe the neutrino fluxes distributions in our Monte Carlo simulations. For each flux and each composition choice, the mean value $\mu$ and standard deviation $\sigma$ are given. Fluxes are in the same units as in Table 6.

$11 \%$, depending on what assumptions we make regarding the heavy element abundances. The SNO neutral current measurements have an accuracy of $\pm 7 \%$ (Ahmed et al. 2004; Aharmim et al. 2005). If one includes all of the solar neutrino and reactor data and the luminosity constraint, then the ${ }^{8} \mathrm{~B}$ neutrino flux is determined to $\pm 5 \%$ (Bahcall et al. 2004a). The theoretical uncertainty for the ${ }^{8} \mathrm{~B}$ solar neutrino flux is more than a factor of 2 larger than the experimental measurement error.

The two largest contributors to the total uncertainty in the predicted ${ }^{8} \mathrm{~B}$ neutrino flux are the cross section factor, $S_{34}$, for the reaction ${ }^{3} \mathrm{He}\left({ }^{4} \mathrm{He}, \gamma\right){ }^{7} \mathrm{Be}$ (which contributes about $7.5 \%$ uncertainty $1 \sigma, \mathrm{BP} 04$ ) and the heavy element abundances (which contribute about $12 \%$ for GS98 abundances and conservative uncertainties and about 5\% for AGS05 abundances and optimistic uncertainties).

\subsection{The ${ }^{7}$ Be Neutrino Flux}

The three panels on the right-hand side of Figure 6 show the histograms of the computed ${ }^{7} \mathrm{Be}$ neutrino flux for the three cases we are considering. We find that the ${ }^{7} \mathrm{Be}$ neutrino flux distribution for each composition choice can be described by a normal distribution, the parameters of which are given in each panel in the figure and in Table 13. The $1 \sigma$ deviation is, as shown in Figure 6 and summarized in Table 15, practically the same for all three cases and ranges from $9.3 \%$ for the AGS05 composition and optimistic uncertainties to $10.5 \%$ for the GS98 conservative case. The theoretical uncertainty for the ${ }^{7} \mathrm{Be}$ solar neutrino flux is relatively insensitive to the assumptions made regarding heavy element abundances and their uncertainties.

The cross section factor $S_{34}$ contributes the largest amount, $\sim 8 \%$, to the total computed uncertainty of the ${ }^{7} \mathrm{Be}$ neutrino flux. This uncertainty could be reduced by improved laboratory measurements (see, e.g., Singh et al. 2004).
The ${ }^{7} \mathrm{Be}$ neutrino flux will be measured by the Borexino solar neutrino experiment (Alimonti et al. 2002) and hopefully also the KamLAND experiment (Araki et al. 2005). In this connection, it is useful to analyze all of the available solar and reactor neutrino data treating the solar neutrino fluxes as unknown variables and including the effects of the luminosity constraint (Bahcall 2002; Spiro \& Vignaud 1990). When this is done, the constraint on the ${ }^{7} \mathrm{Be}$ neutrino flux is (see Table 3 of Bahcall et al. 2004a)

$\phi\left({ }^{7} \mathrm{Be}\right)=1.03_{-1.03}^{+0.24} \phi\left({ }^{7} \mathrm{Be}\right)_{\mathrm{BP} 04}$, exp. data + luminosity constraint.

The ratio of the $\mathrm{BP} 04$ prediction for the ${ }^{7} \mathrm{Be}$ flux to that predicted by the $\mathrm{BSB}(\mathrm{GS} 98$ ) model in this paper is 4.86/4.84 = 1.004 (cf. the ${ }^{7} \mathrm{Be}$ flux in Table 6 of this paper to the ${ }^{7} \mathrm{Be}$ flux in Table 1 of BP04). The coefficient on the right-hand side of equation (37) should be multiplied by 1.004 when the basis for the rate calculation is the $\mathrm{BSB}(\mathrm{GS} 98)$ solar model discussed in this paper.

Unlike the situation with regard to the ${ }^{8} \mathrm{~B}$ neutrino flux for which the experimental error is less than the theoretical uncertainty, the current experimental constraints on the ${ }^{7} \mathrm{Be}$ neutrino flux are much less stringent than the theoretical uncertainty in the predicted rate. If all the experimental evidence is combined with the best solar model prediction, then the uncertainty in the predicted rate for the $\nu+e$ scattering experiments is $\pm 3 \%$ (Bahcall et al. 2004a). This uncertainty has to be combined with a realistic uncertainty of the solar model predictions of the ${ }^{7} \mathrm{Be}$ flux, which we show in this paper is on the order of $\pm 10 \%$.

\subsection{The p-p and pep Neutrino Fluxes}

We begin in $\S 8.3 .1$ by discussing the distribution of the solar model values for the fundamental $p-p$ solar neutrino flux and then describe in $\S 8.3 .2$ the distribution of the closely related pep neutrino flux. The histograms of both the $p-p$ and the pep fluxes are shown in Figure 7. In all cases we find the fluxes in our simulations to be normally distributed.

\subsubsection{The p-p Neutrino Flux}

The left-hand panels of Figure 7 show the histograms of the $p-p$ neutrino fluxes for the indicated three assumptions regarding heavy element composition and their uncertainties. In all three cases, the standard deviation of the theoretical prediction, $\sigma(p-p)$, is about $1 \%$. Moreover, the difference between the best estimate flux that was computed assuming the GS98 composition and the best estimate flux that was computed assuming the AGS05 composition is also 1\% (see Table 6).

TABLE 14

Neutrino Fluxes with Lognormal Distributions

\begin{tabular}{|c|c|c|c|c|c|c|c|c|c|c|c|c|}
\hline \multirow[b]{2}{*}{$\begin{array}{c}\text { FLUX } \\
\text { (1) }\end{array}$} & \multicolumn{4}{|c|}{ GS98-Cons } & \multicolumn{4}{|c|}{ AGS05-OPT } & \multicolumn{4}{|c|}{ AGS05-CONS } \\
\hline & $\begin{array}{c}m \\
(2)\end{array}$ & $\begin{array}{c}s \\
(3)\end{array}$ & $\frac{Q}{(4)}$ & $\begin{array}{c}\mu \\
(5)\end{array}$ & $\begin{array}{c}m \\
(6)\end{array}$ & $\begin{array}{c}s \\
(7)\end{array}$ & $\underset{(8)}{Q}$ & $\begin{array}{c}\mu \\
(9)\end{array}$ & $\begin{array}{c}m \\
(10)\end{array}$ & $\begin{array}{c}s \\
(11)\end{array}$ & $\underset{(12)}{Q}$ & $\begin{array}{c}\mu \\
(13)\end{array}$ \\
\hline${ }^{8} \mathrm{~B}$ & 1.728 & 0.157 & 5.49 & 5.70 & 1.495 & 0.119 & 4.40 & 4.49 & 1.490 & 0.148 & 4.34 & 4.49 \\
\hline${ }^{13} \mathrm{~N}$ & 1.070 & 0.299 & 2.67 & 3.05 & 0.684 & 0.134 & 1.95 & 2.00 & 0.644 & 0.292 & 1.75 & 1.99 \\
\hline${ }^{15} \mathrm{O}$ & 0.792 & 0.304 & 2.01 & 2.31 & 0.350 & 0.151 & 1.39 & 1.44 & 0.312 & 0.295 & 1.25 & 1.43 \\
\hline${ }^{17} \mathrm{~F}$ & 1.674 & 0.486 & 4.21 & 6.00 & 1.166 & 0.152 & 3.14 & 3.25 & 1.105 & 0.466 & 2.43 & 3.37 \\
\hline
\end{tabular}

Notes. - Characterization of the lognormal distributions that describe the neutrino fluxes distributions in our Monte Carlo simulations. For each flux and each

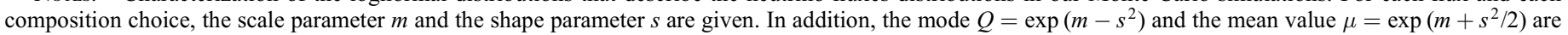
also given. Fluxes are in the same units as in Table 6. 

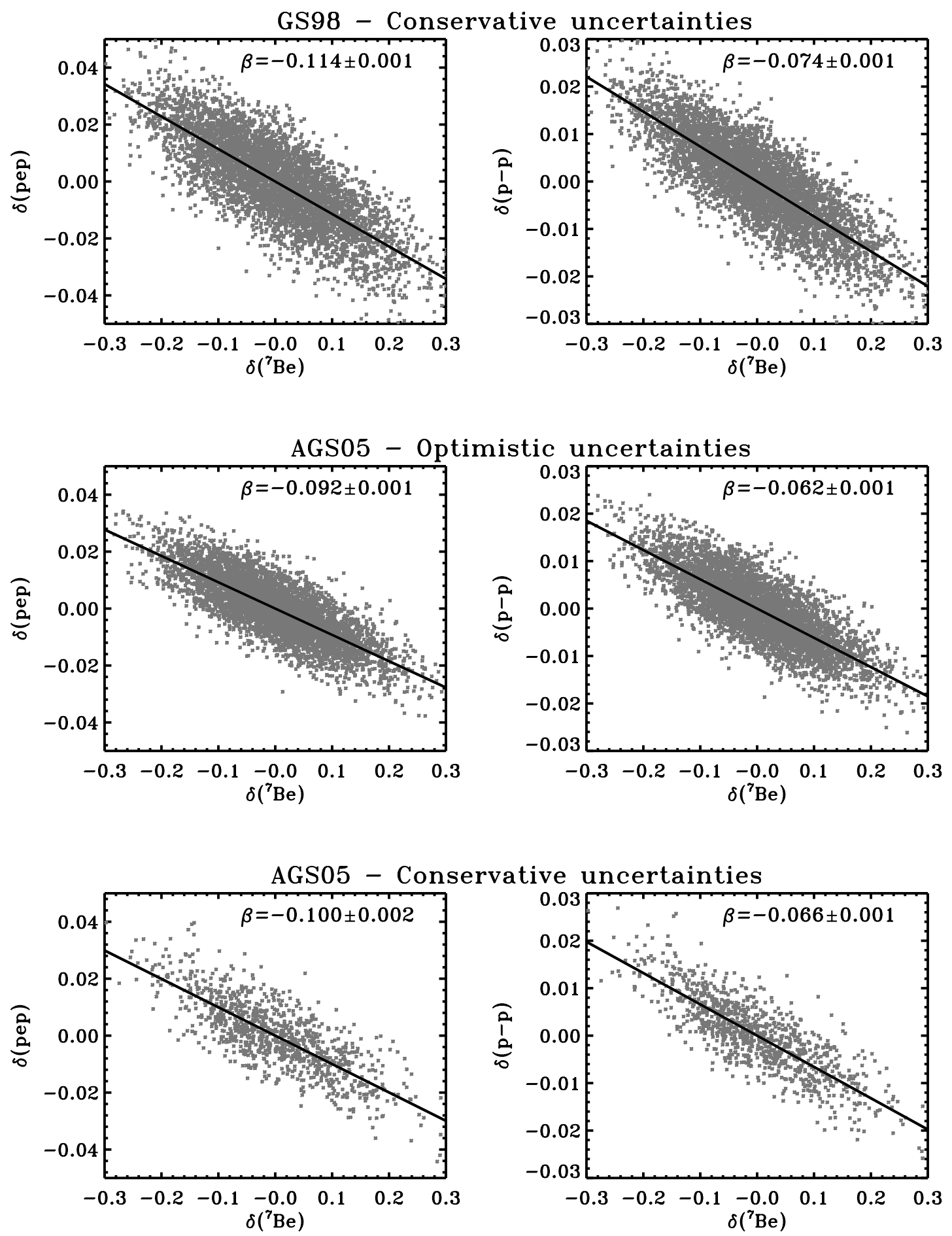

Fig. 8.-The $p e p$ vs. ${ }^{7} \mathrm{Be}$ and the $p-p$ vs. ${ }^{7} \mathrm{Be}$ anticorrelations. The two fluxes are anticorrelated since only one $p-p$ (or $p e p$ ) neutrino is produced if hydrogen burning proceeds through the ${ }^{3} \mathrm{He}\left({ }^{4} \mathrm{He}, \gamma\right)^{7} \mathrm{Be}$ reaction, whereas hydrogen burning in which ${ }^{7} \mathrm{Be}$ is not involved creates two $p-p$ neutrinos (or occasionally pep neutrinos).

The $p$ - $p$ neutrino flux is rather well determined by the existing solar and reactor experiments plus the luminosity constraint (Bahcall 2002). The available data constrain the $p$ - $p$ flux to (see Table 3 of Bahcall et al. 2004a)

$\phi(p-p)=1.01_{-0.02}^{+0.02} \phi(p-p)_{\mathrm{BP} 04}$, exp. data + luminosity constraint.

The ratio of the $\mathrm{BP} 04$ prediction for the $p$ - $p$ flux to that of the $\mathrm{BSB}(\mathrm{GS} 98)$ model flux in this paper is 5.94/5.99 $=0.992$ (cf. the $p-p$ flux in Table 6 of this paper to the flux in Table 1 of BP04). Hence, the coefficient on the right-hand side of equation (38) should be multiplied by 0.992 when the basis for the rate calculation is the currently preferred solar model with GS98 abundances. It should be stressed that the constraint on the $p-p$ neutrino flux that is given in equation (38) is somewhat indirect. Of the solar neutrino experiments performed so far, only the gallium radiochemical experiments (see, e.g., Hampel et al. 1999; Abdurashitov et al. 2002; Altmann et al. 2005) provide measurement constraints on the $p$ - $p$ flux and the gallium measurements do not give a unique flux since neutrino energies are 
TABLE 15

Total Percent $1 \sigma$ Deviations in Neutrino Fluxes due to All Sources

\begin{tabular}{|c|c|c|c|c|c|c|c|c|c|}
\hline \multirow[b]{2}{*}{$\begin{array}{c}\text { FLUX } \\
(1)\end{array}$} & \multicolumn{3}{|c|}{ GS98-Cons } & \multicolumn{3}{|c|}{ AGS05-Opt } & \multicolumn{3}{|c|}{ AGS05-Cons } \\
\hline & $\begin{array}{c}\sigma \\
(\%) \\
(2)\end{array}$ & $\begin{array}{c}\sigma_{+} \\
(\%) \\
(3)\end{array}$ & $\begin{array}{c}\sigma_{-} \\
(\%) \\
(4)\end{array}$ & $\begin{array}{c}\sigma \\
(\%) \\
(5)\end{array}$ & $\begin{array}{c}\sigma_{+} \\
(\%) \\
(6)\end{array}$ & $\begin{array}{c}\sigma_{-} \\
(\%) \\
(7)\end{array}$ & $\begin{array}{c}\sigma \\
(\%) \\
(8)\end{array}$ & $\begin{array}{c}\sigma_{+} \\
(\%) \\
(9)\end{array}$ & $\begin{array}{c}\sigma_{-} \\
(\%) \\
(10)\end{array}$ \\
\hline$p-p \ldots \ldots \ldots \ldots$ & 0.9 & & & 0.7 & & & 0.8 & & \\
\hline pep .............. & 1.5 & & & 1.1 & & & 1.3 & & \\
\hline hep ............... & 15.5 & & & 15.5 & & & 15.3 & & \\
\hline${ }^{7} \mathrm{Be} . \ldots \ldots \ldots \ldots$ & 10.5 & & & 9.3 & & & 10.3 & & \\
\hline & & 17.3 & 14.7 & & 12.7 & 11.3 & & 16.1 & 14.1 \\
\hline${ }^{13} \mathrm{~N} \ldots \ldots \ldots \ldots$ & & 36.6 & 26.8 & & 14.5 & 12.7 & & 35.5 & 26.2 \\
\hline${ }^{15} \mathrm{O} \ldots \ldots \ldots \ldots$ & & 37.4 & 27.2 & & 16.5 & 14.2 & & 36.1 & 26.5 \\
\hline${ }^{17} \mathrm{~F} \ldots \ldots \ldots \ldots$ & & 72.4 & 42.0 & & 16.6 & 14.2 & & 67.6 & 40.4 \\
\hline
\end{tabular}

Notes.-For the neutrino flux $p$ - $p$, pep, hep, and ${ }^{7} \mathrm{Be}$ neutrino fluxes the total $1 \sigma$ uncertainty is given in $\%$ of the mean values of each flux distribution listed in Table 13. The same results are obtained if the best estimate neutrino fluxes, listed in col. (2) of Table 6, are used. Cols. (2)-(10) correspond to the different composition choices described in the text. For fluxes with lognorma distributions, ${ }^{8} \mathrm{~B},{ }^{13} \mathrm{~N},{ }^{15} \mathrm{O}$, and ${ }^{17} \mathrm{~F}$, the uncertainties $\sigma_{+}$and $\sigma_{-}$that define the $1 \sigma$ confidence level are given separately. A detailed definition of $\sigma_{+}$and $\sigma_{-}$is given in the Appendix. Relative uncertainties are computed with respect to the most probable value $Q$ of each distribution, given in Table 14. Table 3 gives the numerical values for conservative and optimistic abundance uncertainties.

not measured. For the gallium experiments, all neutrinos above a fixed threshold are counted. Moreover, the luminosity constraint is critical for obtaining bounds on the $p$ - $p$ flux; without the luminosity constraint, the allowed range of the $p$ - $p$ flux is very large, much larger than the theoretical uncertainty (Bahcall et al. 2004a). At present, the accuracy of the experimental determination of the $p$ - $p$ flux when supplemented with the luminosity constraint, is comparable to the theoretical uncertainty in the prediction of this flux.

The American Physical Society multidivisional neutrino study recommended a precision measurement of the $p-p$ neutrino flux to, among other things, "test our understanding of how neutrinos change flavor, probe the fundamental question of whether the Sun shines only through nuclear fusion" (see Freedman \& Kayser 2004). Since the $p$ - $p$ reaction initiates, according to the standard solar model, more than $99 \%$ of the nuclear energy generation in the Sun (see Table 6), an accurate direct measurement of the $p-p$ flux is of great importance for testing the widely used theory of stellar evolution.

A number of promising approaches to measuring the $p$ - $p$ neutrino flux are in various stages of development (Raghavan 1976, 2001; Gorodetsky et al. 1999; Ejiri et al. 2000; Suzuki et al. 2000; McKinsey \& Doyle 2000; McKinsey \& Coakley 2005; Nakahata 2001; McDonald 2004; Suzuki 2005; Oberauer et al. 2005; Dolbeau et al. 2005; Lanou 2005).

\subsubsection{The pep Neutrino Flux}

The three right panels of Figure 7 present the histograms of the calculated flux of pep solar neutrinos. The standard deviation of the flux, $\sigma_{p e p}$, varies between $1.1 \%$ and $1.5 \%$, depending on which heavy element abundances and uncertainties are adopted. There is no existing significant experimental constraint on the pep flux, which is about 400 times smaller than the $p p$ flux.

However, the monoenergetic pep neutrinos have an energy of $1.4 \mathrm{MeV}$, compared to the maximum energy of $0.43 \mathrm{MeV}$ of the $p-p$ neutrinos. Therefore, it is possible that the pep neutrinos

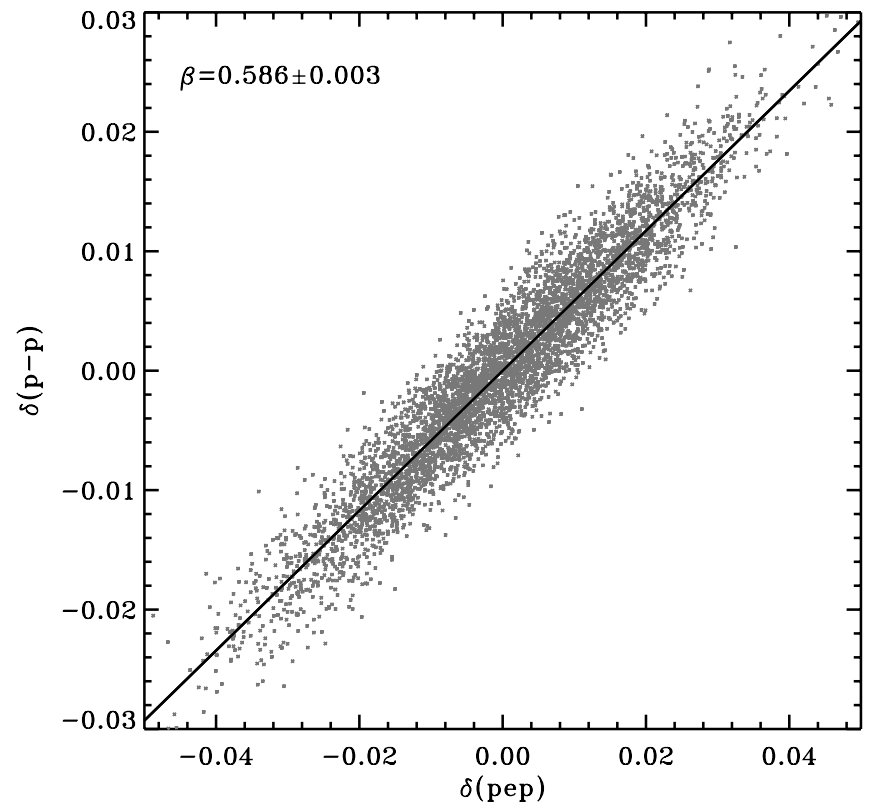

FIG. 9.-Correlation between the $p-p$ and pep fluxes. The two fluxes are approximately proportional to each other since they share the same nuclear matrix element (Bahcall \& May 1969).

could be measured in an $\nu+e$ scattering experiment like Borexino (Alimonti et al. 2002) or KamLAND (Araki et al. 2005).

\subsubsection{The pep versus ${ }^{7} \mathrm{Be}$ and $p$-p versus ${ }^{7} \mathrm{Be}$ Correlations}

We know from general considerations of the reactions in the $p$ - $p$ chain that the $p$ - $p$ and ${ }^{7} \mathrm{Be}$ solar neutrino fluxes are inversely correlated. If the $p$ - $p$ chain is terminated by the ${ }^{3} \mathrm{He}-{ }^{3} \mathrm{He}$ reaction, then two $p-p$ neutrinos, and no ${ }^{7} \mathrm{Be}$ neutrinos, are produced. If the $p$ - $p$ chain is terminated by the ${ }^{3} \mathrm{He}-{ }^{4} \mathrm{He}$ reaction, then one $p-p$ neutrino and one ${ }^{7} \mathrm{Be}$ neutrino (nearly always) is produced (only rarely is the ${ }^{7} \mathrm{Be}$ neutrino replaced by a ${ }^{8} \mathrm{~B}$ neutrino). Moreover, the pep flux is very nearly proportional to the $p$ - $p$ flux (Bahcall \& May 1969) and can be used as a surrogate for the $p$ - $p$ flux in the above discussion.

It is possible that both the pep neutrino flux and the ${ }^{7} \mathrm{Be}$ neutrino flux will be measured in the next few years in the existing Borexino solar neutrino experiment (Alimonti et al. 2002). For a discussion of this possibility, the reader is referred to the paper by Galbiati et al. (2005). In any event, we can look forward to the measurement of the $p-p$ neutrino flux in one of the solar neutrino experiments currently being developed for this purpose (see, e.g., the discussions by Raghavan 1976, 2001; Gorodetsky et al. 1999; Ejiri et al. 2000; Suzuki et al. 2000; McKinsey \& Doyle 2000; McKinsey \& Coakley 2005; Nakahata 2001; McDonald 2004; Suzuki 2005; Oberauer et al. 2005; Lanou 2005).

If either the pep or the $p$ - $p$ neutrino flux is measured and the ${ }^{7} \mathrm{Be}$ neutrino flux is also determined experimentally, then one can test directly a fundamental prediction of stellar evolution theory. The prediction to be tested is the anticorrelation between the $p$ - $p$ (or $p e p$ ) neutrino flux and the ${ }^{7} \mathrm{Be}$ neutrino flux. In what follows, we suppose for specificity that the pep neutrino flux is measured before the $p$ - $p$ flux and therefore we first explore the anticorrelation between the pep and ${ }^{7} \mathrm{Be}$ neutrino fluxes. The calculational steps involved in determining the anticorrelation are identical for $p e p$ versus ${ }^{7} \mathrm{Be}$ and $p-p$ versus ${ }^{7} \mathrm{Be}$. We will present results for both cases. 
TABLE 16

Correlation Coefficients for 5000 Sets of Neutrino Fluxes: Grevesse \& Sauval (1998)

Heavy Element Abundances and Conservative Uncertainties

\begin{tabular}{|c|c|c|c|c|c|c|c|c|}
\hline $\begin{array}{c}\text { Flux } \\
\text { (1) }\end{array}$ & $\begin{array}{l}p p \\
(2)\end{array}$ & $\begin{array}{r}\text { pep } \\
\text { (3) }\end{array}$ & $\begin{array}{l}\text { hep } \\
\text { (4) }\end{array}$ & $\begin{array}{l}{ }^{7} \mathrm{Be} \\
(5)\end{array}$ & $\begin{array}{l}{ }^{8} \mathrm{~B} \\
(6)\end{array}$ & $\begin{array}{l}{ }^{13} \mathrm{~N} \\
(7)\end{array}$ & $\begin{array}{l}{ }^{15} \mathrm{O} \\
(8)\end{array}$ & $\begin{array}{l}{ }^{17} \mathrm{~F} \\
(9)\end{array}$ \\
\hline$p-p$ & 1.000 & 0.954 & 0.082 & -0.819 & -0.720 & -0.349 & -0.381 & -0.319 \\
\hline 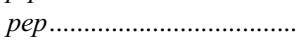 & 0.954 & 1.000 & 0.087 & -0.780 & -0.730 & -0.407 & -0.439 & -0.369 \\
\hline 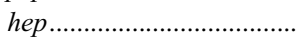 & 0.082 & 0.087 & 1.000 & -0.062 & -0.086 & -0.052 & -0.058 & -0.076 \\
\hline${ }^{7} \mathrm{Be}$ & -0.819 & -0.780 & -0.062 & 1.000 & 0.887 & 0.154 & 0.204 & 0.332 \\
\hline${ }^{8} \mathrm{~B}$ & -0.720 & -0.730 & -0.086 & 0.887 & 1.000 & 0.269 & 0.333 & 0.486 \\
\hline${ }^{13} \mathrm{~N}$ & -0.349 & -0.407 & -0.052 & 0.154 & 0.269 & 1.000 & 0.991 & 0.172 \\
\hline${ }^{15} \mathrm{O}$ & -0.381 & -0.439 & -0.058 & 0.204 & 0.333 & 0.991 & 1.000 & 0.219 \\
\hline${ }^{17} \mathrm{~F}$ & -0.319 & -0.369 & -0.076 & 0.332 & 0.486 & 0.172 & 0.219 & 1.000 \\
\hline
\end{tabular}

Notes.-The correlation coefficients in the table are defined by eq. (50). The fluxes used to evaluate the coefficients were calculated using solar models that incorporated Grevesse \& Sauval (1998) surface heavy element abundances and conservative uncertainties.

How can we determine quantitatively the $p e p$ versus ${ }^{7} \mathrm{Be}$ anticorrelation? To answer this question it is convenient to define dimensionless variables $\delta($ pep $)$ and $\delta\left({ }^{7} \mathrm{Be}\right)$ by the relations

$$
\delta(\text { pep })=\frac{\phi(p e p)-\mu(\text { pep })}{\mu(\text { pep })} ; \quad \delta\left({ }^{7} \mathrm{Be}\right)=\frac{\phi\left({ }^{7} \mathrm{Be}\right)-\mu\left({ }^{7} \mathrm{Be}\right)}{\mu\left({ }^{7} \mathrm{Be}\right)},
$$

where $\phi(p e p)$ is the pep flux from a single solar model and $\mu($ pep $)$ is the mean pep flux in our simulations given in Table 13 [with analogous definitions for $\delta\left({ }^{7} \mathrm{Be}\right), \phi\left({ }^{7} \mathrm{Be}\right)$, and $\left.\mu\left({ }^{7} \mathrm{Be}\right)\right]$.

Figure 8 shows in the three left panels the solar model prediction for the anticorrelation between $\delta(p e p)$ and $\delta\left({ }^{7} \mathrm{Be}\right)$. For the top left panel, the GS98-Cons case, the best-fit straight line of the form

$$
\delta(p e p)=\beta\left(p e p,{ }^{7} \mathrm{Be}\right) \delta\left({ }^{7} \mathrm{Be}\right)
$$

computed by a least-squares fitting, has a slope

$$
\beta\left(p e p,{ }^{7} \mathrm{Be}\right)=-0.114 \pm 0.001 .
$$

The residuals

$$
R\left(p e p,{ }^{7} \mathrm{Be}\right)=\delta(\text { pep })-\beta\left(\text { pep },{ }^{7} \mathrm{Be}\right) \delta\left({ }^{7} \mathrm{Be}\right)
$$

are normally distributed with a standard deviation

$$
\sigma\left(p e p ;{ }^{7} \mathrm{Be}\right)=0.0096 \text {. }
$$

If one measures precisely the ${ }^{7} \mathrm{Be}$ neutrino flux, then our current knowledge of solar model determines the expected pep neutrino flux to an accuracy of $0.96 \%$ at the $1 \sigma$ level. The results are similar for the other two cases shown in Figure 8. For the AGS05-Opt case we find $\beta\left(p e p,{ }^{7} \mathrm{Be}\right)=-0.092 \pm 0.001$ and $\sigma\left(\right.$ pep $\left.;{ }^{7} \mathrm{Be}\right)=0.0066$, while for AGS05-Cons we get $\beta\left(\right.$ pep, $\left.{ }^{7} \mathrm{Be}\right)=-0.100 \pm 0.0024$ and $\sigma\left(\right.$ pep $\left.;{ }^{7} \mathrm{Be}\right)=0.0079$.

The right-hand panels of Figure 8 show the anticorrelation between the $p$ - $p$ and the ${ }^{7} \mathrm{Be}$ neutrino fluxes. For the top right panel, GS98-Cons composition choice, the best-fit straight line has a slope

$$
\beta\left(p p,{ }^{7} \mathrm{Be}\right)=-0.0736 \pm 0.0007 .
$$

The corresponding $1 \sigma$ uncertainty in predicting $\delta(p p)$ from a known value of the ${ }^{7} \mathrm{Be}$ neutrino flux is

$$
\sigma\left(p-p ;{ }^{7} \mathrm{Be}\right)=0.0054 .
$$

Thus, one can predict the $p-p$ flux to an accuracy of about $0.54 \%$ from a precisely measured value of the ${ }^{7} \mathrm{Be}$ flux. The $p-p$ versus ${ }^{7} \mathrm{Be}$ anticorrelation is somewhat tighter than the $p e p$ versus ${ }^{7} \mathrm{Be}$ anticorrelation.

Similar values are obtained for the other two cases. We find, respectively, $\beta\left(p p,{ }^{7} \mathrm{Be}\right)=-0.0617 \pm 0.0006$ and $\sigma\left(p-p ;{ }^{7} \mathrm{Be}\right)=$ 0.0044 and $\beta\left(p p,{ }^{7} \mathrm{Be}\right)=-0.0659 \pm 0.0014$ and $\sigma\left(p-p ;{ }^{7} \mathrm{Be}\right)=$ 0.0046 for the AGS05-Opt and AGS05-Cons cases, respectively.

If one is interested in the inverse correlations, e.g., $\delta\left({ }^{7} \mathrm{Be}\right)=$ $\beta\left({ }^{7} \mathrm{Be}, p e p\right) \delta(p e p)$, they can be easily obtained by recalling that given two quantities $x$ and $y$, then if $x=\beta(x, y) y$ and $y=$ $\beta(y, x) x$, where $\beta(x, y)$ and $\beta(y, x)$ are computed from leastsquares fitting, then they satisfy the relation

$$
\beta(x, y) \beta(y, x)=\rho^{2}(x, y),
$$

where $\rho(x, y)$ is the correlation coefficient between $x$ and $y$. The correlation coefficients of the neutrino fluxes are discussed in $\S 9$ and summarized in Tables 16 and 17.

If our general picture of how nuclear fusion reactions occur in the solar interior is correct, then measurements of the pep (or $p-p)$ and the ${ }^{7} \mathrm{Be}$ neutrino fluxes must lie on one of the very similar lines shown in Figure 8. The values given in this subsection for $\beta\left(\right.$ pep,$\left.{ }^{7} \mathrm{Be}\right)$ represent a fundamental and testable prediction of the theory of nuclear energy generation in stars. They encapsulate the competition in the solar interior between the two primary branches, $p-p(\mathrm{I})$ and $p-p(\mathrm{II})$, of the $p-p$ chain.

\subsubsection{The pep versus $p$-p Correlation}

What is the relation between the production of the $p-p$ and the pep neutrinos? They share the same nuclear matrix element and differ in a multiplicative factor that depends approximately on the electron number density divided by the square root of the ambient temperature (Bahcall \& May 1969). This factor does not change very much from one solar model to the next. As a result, the $p e p$ rate is very nearly proportional to the $p-p$ rate.

Suppose the pep flux is measured before the $p$ - $p$ flux is determined by a direct experiment. How accurately can one infer the $p-p$ neutrino flux if one measures the pep flux? To answer this question, we plot $\delta(p e p)$ versus $\delta(p-p)$, where the meaning of the operator $\delta$ is defined in equation (39). 
TABLE 17

Correlation Coefficients for Neutrino Fluxes: Asplund et al. (2005) Heavy Element Abundances and Optimistic Uncertainties

\begin{tabular}{|c|c|c|c|c|c|c|c|c|}
\hline $\begin{array}{c}\text { Flux } \\
\text { (1) }\end{array}$ & $\begin{array}{l}p p \\
(2)\end{array}$ & $\begin{array}{l}\text { pep } \\
(3)\end{array}$ & $\begin{array}{l}\text { hep } \\
(4)\end{array}$ & $\begin{array}{l}{ }^{7} \mathrm{Be} \\
(5)\end{array}$ & $\begin{array}{l}{ }^{8} \mathrm{~B} \\
(6)\end{array}$ & $\begin{array}{l}{ }^{13} \mathrm{~N} \\
(7)\end{array}$ & $\begin{array}{l}{ }^{15} \mathrm{O} \\
(8)\end{array}$ & $\begin{array}{l}{ }^{17} \mathrm{~F} \\
(9)\end{array}$ \\
\hline$p-p$ & 1.000 & 0.967 & -0.012 & -0.796 & -0.642 & -0.127 & -0.132 & -0.111 \\
\hline 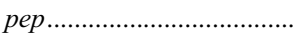 & 0.967 & 1.000 & 0.001 & -0.793 & -0.667 & -0.162 & -0.171 & -0.137 \\
\hline 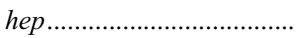 & -0.012 & 0.001 & 1.000 & 0.022 & 0.021 & -0.005 & -0.008 & -0.014 \\
\hline${ }^{7} \mathrm{Be}$ & -0.796 & -0.793 & 0.022 & 1.000 & 0.878 & 0.125 & 0.155 & 0.237 \\
\hline${ }^{8} \mathrm{~B} \ldots \ldots \ldots$ & -0.642 & -0.667 & 0.021 & 0.878 & 1.000 & 0.257 & 0.296 & 0.412 \\
\hline${ }^{13} \mathrm{~N}$ & -0.127 & -0.162 & -0.005 & 0.125 & 0.257 & 1.000 & 0.984 & 0.299 \\
\hline${ }^{15} \mathrm{O}$ & -0.132 & -0.171 & -0.008 & 0.155 & 0.296 & 0.984 & 1.000 & 0.338 \\
\hline${ }^{17} \mathrm{~F}$ & -0.111 & -0.137 & -0.014 & 0.237 & 0.412 & 0.299 & 0.338 & 1.000 \\
\hline
\end{tabular}

Notes.-The correlation coefficients in the table are defined by eq. (50). The fluxes used to create evaluate the coefficients were calculated using Asplund et al. (2005) surface heavy element abundances and optimistic uncertainties.

Figure 9 shows the rather tight correlation between $\delta(p e p)$ and $\delta(p-p)$. For the GS98-Cons case, the best-fit straight line of the form

$$
\delta(p-p)=\beta(p-p, p e p) \delta(p e p)
$$

has a slope

$$
\beta(p-p, p e p)=0.586 \pm 0.003,
$$

and

$$
\sigma(p-p, p e p)=0.0028 \text {. }
$$

Thus, the $p-p$ neutrino flux can be inferred from a precisely measured pep neutrino flux to an accuracy of about $0.3 \%$. The results are similar for the other two cases we are considering. For the AGS05-Opt case, we find $\beta(p-p, p e p)=0.642 \pm 0.002$ and $\sigma(p-p, p e p)=0.0019$. Similarly, for AGS05-Cons we find $\beta(p-p, p e p)=0.598 \pm 0.007$, while $\sigma(p-p, p e p)=0.0027$.

In summary, the $p$ - $p$ flux can be inferred from a precisely measured pep flux to an accuracy of $0.25 \% \pm 0.05 \%$, depending on which heavy element abundances are adopted and which abundance uncertainties are used. From the inverse correlation, the $p e p$ flux can be inferred from a precisely measured $p$ - $p$ flux to an accuracy of $0.39 \% \pm 0.09 \%$.

\subsection{The hep, ${ }^{13} \mathrm{~N},{ }^{15} \mathrm{O}$, and ${ }^{17} \mathrm{~F}$ Neutrino Fluxes}

Figure 10 shows, for GS98 abundances and conservative uncertainties, the histograms of the number of solar models with different values of the hep, ${ }^{13} \mathrm{~N},{ }^{15} \mathrm{O}$, and ${ }^{17} \mathrm{~F}$ solar neutrino fluxes. While the hep flux is normally distributed $(99.9 \%$ confidence level), the three CNO fluxes follow lognormal distributions (better than $95 \%$ confidence level). The results for all three composition cases that we are considering are summarized in Tables 13, 14 , and 15 .

The total uncertainty for the hep neutrino flux is dominated by the $15.1 \%$ uncertainty (Park et al. 2003) from the calculation of the nuclear matrix element. All other sources of uncertainty contribute less than or on the order of $2 \%$. Therefore, the calculated standard deviations for the hep neutrinos are essentially independent of the adopted heavy element abundances and their uncertainties.

For the ${ }^{13} \mathrm{~N},{ }^{15} \mathrm{O}$, and ${ }^{17} \mathrm{~F}$ solar neutrino fluxes, the standard deviations are dominated by composition uncertainties if we adopt conservative uncertainties. The marked asymmetry of the distributions of the CNO neutrino fluxes is apparent in Figure 10.
This asymmetry reflects the lognormal distribution for composition uncertainties that we have adopted and discussed in $\S 2.3$. If we use optimistic composition uncertainties, the cross section factor $S_{1,14}$ for the ${ }^{14} \mathrm{~N}(p, \gamma){ }^{15} \mathrm{O}$ reaction $(8.4 \%$ uncertainty) and the composition uncertainties make comparable contributions to the ${ }^{13} \mathrm{~N}$ and ${ }^{15} \mathrm{O}$ neutrino fluxes uncertainties.

There are no funded experiments for which the detection of the hep, ${ }^{13} \mathrm{~N},{ }^{15} \mathrm{O}$, and ${ }^{17} \mathrm{~F}$ solar neutrino fluxes seems likely if the fluxes and their uncertainties calculated in this paper are correct.

\section{NEUTRINO FLUX CORRELATION COEFFICIENTS}

We have seen in $\S 8$ that some of the neutrino fluxes are highly correlated. These correlations are exhibited in, e.g., Figure 8, which illustrates the anticorrelation of the pep and $p p$ fluxes with the ${ }^{7} \mathrm{Be}$ flux, and Figure 9 , which shows the correlation of the pep and the $p p$ fluxes. These correlations play an important role in global analysis of solar neutrino experiments (see, e.g., Fogli \& Lisi 1995; Bahcall et al. 2001a; Fogli et al. 2002).

The correlations of the fluxes arise from two sources: (1) the solution of the equations of stellar evolution; and (2) the effects of changes in individual input parameters.

In the past, the correlations of solar neutrino fluxes have been taken into account by using the logarithmic partial derivatives of individual neutrino fluxes with respect to nine input parameters (Bahcall \& Ulrich 1988; Bahcall 1989). The standard treatment of the flux correlations, when represented by partial derivatives of fluxes with respect to input parameters, is contained in the important paper by Fogli \& Lisi (1995).

In this section, we use the results of our Monte Carlo simulations to derive the correlations due to all 21 input parameters and to the solution of the equations of stellar evolution. By directly calculating the correlation coefficients among the Monte Carlo neutrino fluxes, we are able to present a simple and complete summary of the correlations. These results will enable simpler and more accurate theoretical analysis of solar neutrino oscillations.

We summarize the correlations in terms of the correlation coefficients, $\rho(i, j)$, defined in the usual way by

$$
\rho(i, j)=\frac{N^{-1} \sum_{n=1}^{N} \Delta \phi_{i}^{n} \Delta \phi_{j}^{n}}{\sigma_{i} \sigma_{j}}
$$

where $\Delta \phi_{i}^{n}=\phi_{i}^{n}-\phi_{i \text {,average }}, \sigma_{i}$ is the standard deviation of the $i$ th flux type ( $i=p p$, pep, hep, ${ }^{7} \mathrm{Be},{ }^{8} \mathrm{~B},{ }^{13} \mathrm{~N},{ }^{15} \mathrm{O}$, and ${ }^{17} \mathrm{~F}$ ), and $N=5000$ is the total number of cases considered in the separate Monte Carlo simulations that incorporated GS98 or AGS05 

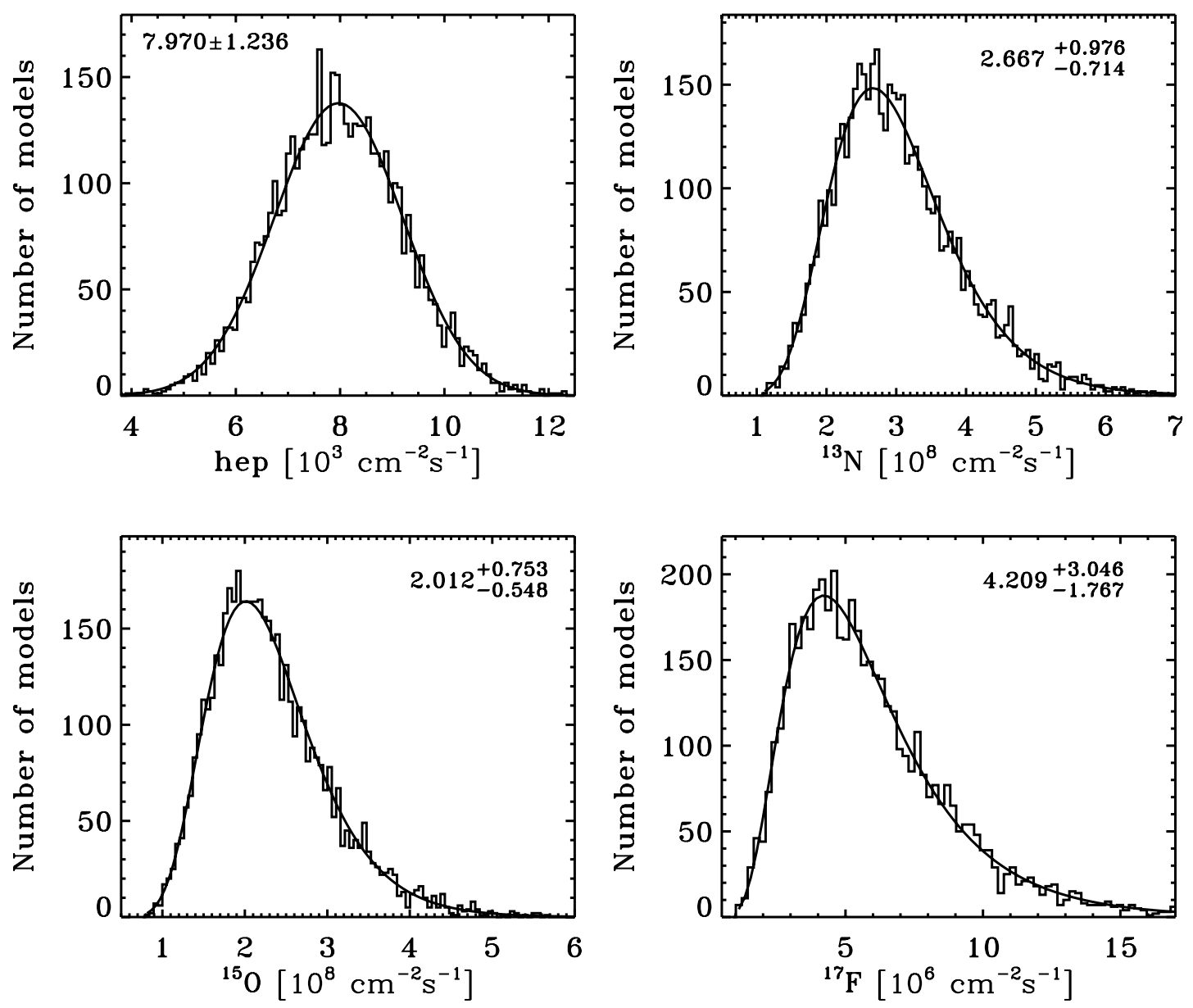

FIG. 10.-The hep, ${ }^{13} \mathrm{~N},{ }^{15} \mathrm{O}$, and ${ }^{17} \mathrm{~F}$ neutrino fluxes from out Monte Carlo simulation with the GS98-Cons composition choice. The hep distribution follows a normal distribution with the parameters shown in the top left panel. The distributions of the CNO fluxes are markedly asymmetric as they reflect the lognormal distribution for the composition uncertainties that is discussed in $\S 2.3$. Results are analogous for the AGS05-Opt and AGS05-Cons composition choices.

heavy element abundances. The correlation matrix is symmetric, $\rho(i, j)=\rho(j, i)$, and has by definition unit diagonal elements.

Tables 16 and 17 present the correlation coefficients calculated with the Monte Carlo simulations that used, respectively, the Grevesse \& Sauval (1998) heavy element abundances with conservative uncertainties and the Asplund et al. (2005) heavy element abundances with optimistic uncertainties.

We see from Tables 16 and 17 that the $p p$ and pep neutrino fluxes are strongly anticorrelated with the ${ }^{7} \mathrm{Be}$ and ${ }^{8} \mathrm{~B}$ neutrino fluxes and mildly anticorrelated with the $\mathrm{CNO}$ neutrino fluxes. The $p p$ and $p e p$ fluxes are, as discussed in $\S 8.3 .4$, very strongly correlated. The ${ }^{7} \mathrm{Be}$ and ${ }^{8} \mathrm{~B}$ neutrino fluxes are strongly correlated, since both are initiated by the same fusion reaction, ${ }^{3} \mathrm{He}\left({ }^{4} \mathrm{He}, \gamma\right){ }^{7} \mathrm{Be}$. Since the ${ }^{7} \mathrm{Be}$ and ${ }^{8} \mathrm{~B}$ fluxes both occur predominantly in higher temperature regions of the Sun (see Fig. 1), where the Gamow penetration factor is more easily overcome, these fluxes are also mildly correlated with the CNO neutrino fluxes that are also mostly produced at higher temperatures. Of course, the ${ }^{13} \mathrm{~N}$ and ${ }^{15} \mathrm{O}$ neutrino fluxes are strongly correlated with each other since they are both involved in the $\mathrm{CN}$ cycle that operates close to steady state in the inner $\left(R<0.12 R_{\odot}\right)$ regions of the Sun.

Comparing the correlation coefficients given in Tables 16 and 17 , we see that the same general trends are obtained independent of which assumption we make regarding the heavy element abundances and their uncertainties. However, there are important quantitative differences. In particular, the correlations involving the CNO neutrino fluxes with fluxes from the $p p$ chain are weaker when the Asplund et al. (2005) abundances and optimistic uncertainties are adopted.

\section{NUCLEAR FUSION FRACTIONS}

For pedagogical purposes and in order to have a conceptual overview of solar energy generation, it is useful to calculate the fraction of the total nuclear energy generation that occurs via each of the most important fusion paths. We present in this section the best estimates (given in the last column of Table 6 for the best current standard solar models) and the $1 \sigma$ uncertainties in the best estimates of the fractions that correspond to different ways of burning hydrogen.

Table 18 gives the fractions of the total nuclear fusion energy generation in standard solar models that are produced by different fusion reaction paths. We present results for all three of the composition options.

More than $99 \%$ of the total nuclear energy generation is produced by the $p-p$ reactions in our solar models, while less than $1 \%$ of the energy is generated by the CNO reactions. These fractions are robust to all the input uncertainties of our standard solar models. The total standard deviations from variations in all the 21 input parameters in the Monte Carlo simulations are between $0.07 \%$ or $0.3 \%$.

About $88 \%$ or $90 \%$ of the energy is derived, on average for our standard models, from reactions that begin with the fundamental $p$ - $p$ reaction and terminate with the ${ }^{3} \mathrm{He}\left({ }^{3} \mathrm{He}, 2 p\right)^{4} \mathrm{He}$ 
TABLE 18

Fractions of Nuclear Energy Generation That Are Produced By Different Reaction Pathways

\begin{tabular}{|c|c|c|c|}
\hline $\begin{array}{l}\text { Fusion Branch } \\
\text { Fraction } \\
\text { (1) }\end{array}$ & $\begin{array}{l}\text { GS98-Cons } \\
\text { (2) }\end{array}$ & $\begin{array}{l}\text { AGS05-Opt } \\
\text { (3) }\end{array}$ & $\begin{array}{c}\text { AGS05-Cons } \\
\text { (4) }\end{array}$ \\
\hline$p-p$ & $99.2 \pm 0.3$ & $99.5 \pm 0.1$ & $99.5 \pm 0.2$ \\
\hline $\mathrm{CNO}$ & $0.8 \pm 0.2$ & $0.5 \pm 0.07$ & $0.5 \pm 0.1$ \\
\hline$p-p(\mathrm{I})$ & $88.3 \pm 1.3$ & $89.6 \pm 1.0$ & $89.6 \pm 1.1$ \\
\hline$p-p($ II $) \ldots \ldots \ldots \ldots \ldots \ldots$ & $10.8 \pm 1.1$ & $9.6 \pm 0.9$ & $9.6 \pm 1.0$ \\
\hline$p-p(\mathrm{III}) \ldots \ldots \ldots \ldots \ldots \ldots$ & $0.9 \pm 0.1$ & $0.8 \pm 0.08$ & $0.8 \pm 0.08$ \\
\hline
\end{tabular}

Notes.-The table presents results for percentages of solar energy generation via different nuclear paths: all $p$ - $p$ reactions (row 1); all CNO reactions (row 2); $p$ - $p$ (I) (terminated by ${ }^{3} \mathrm{He}\left({ }^{3} \mathrm{He}, 2 \mathrm{p}\right){ }^{4} \mathrm{He}$ or $\mathrm{p}\left({ }^{2} \mathrm{H}, \gamma\right){ }^{3} \mathrm{He}$, row 3); $p$ - $p$ (II) (terminated through $e^{-}\left({ }^{7} \mathrm{Be}, \nu_{e}\right)^{7} \mathrm{Li}$, row 4$)$ and $p-p$ (III) (terminated through $p\left({ }^{7} \mathrm{Be}\right.$, $\gamma)^{8} \mathrm{~B}$, row 5). The values in col. (2) correspond to GS98 heavy element abundances and conservative uncertainties, col. (3) corresponds to AGS05 abundances and optimistic uncertainties, and col. (4) corresponds to AGS05 abundances and conservative uncertainties. Table 3 gives the numerical values for conservative and optimistic abundance uncertainties.

reaction $(p-p(\mathrm{I}))$. Nearly all of the remainder of the nuclear energy, $10 \%$ or $11 \%$, is generated in our models by $p-p$ reactions that go through the reaction ${ }^{3} \mathrm{He}\left({ }^{4} \mathrm{He}, \gamma\right){ }^{7} \mathrm{Be}$ that creates ${ }^{7} \mathrm{Be}$ solar neutrinos by electron capture $(p-p(\mathrm{II}))$. These fractions also have relatively small variations (standard deviations) due to the choice of different input parameters. The standard deviations are typically $1 \%$ for the $p-p(\mathrm{I})$ and $p-p(\mathrm{II})$ fractions.

Extraordinary as it seems, most of solar neutrino astronomy so far has focused on the $p$ - $p$ (III) reactions that involve the production of rare ${ }^{8} \mathrm{~B}$ neutrinos. The crucial reaction sequence terminating these $p$ - $p$ reactions consists of the reaction ${ }^{3} \mathrm{He}\left({ }^{4} \mathrm{He}, \gamma\right)^{7} \mathrm{Be}$ followed by ${ }^{7} \mathrm{Be}(p, \gamma){ }^{8} \mathrm{~B}$. Less than $1 \%$ of the energy generation in our solar models corresponds to this rare reaction pathway and the standard deviation of this fraction is only about $0.1 \%$. The Kamiokande (Fukuda et al. 1996), Super-Kamiokande (Fukuda et al. 2001, 2002), and SNO solar neutrino experiments (Ahmed et al. 2004; Aharmim et al. 2005) only detect neutrinos from this rare set of reactions. Moreover, the original chlorine solar neutrino experiment by R. Davis, Jr., and his colleagues (Cleveland et al. 1998) is primarily sensitive to ${ }^{8} \mathrm{~B}$ neutrinos because of a special superallowed transition between ${ }^{37} \mathrm{Cl}$ and ${ }^{37} \mathrm{Ar}$ (Bahcall 1964).

\section{CONCLUSIONS AND DISCUSSION}

We provide in this paper quantitative estimates of the accuracy with which standard solar models predict measurable quantities by means of a Monte Carlo simulation. These estimates provide the most comprehensive summary of solar model predictions and for many of the predicted quantities, e.g., the eight helioseismologically measured quantities $\left(Y_{\text {surf }}, R_{\mathrm{CZ}}\right.$, and the six difference rms) the estimates given here provide the only consistent quantitative estimates of the uncertainties of the predictions.

As of this writing, there is considerable uncertainty regarding the best estimates for the surface chemical composition of the Sun, one of the important sets of input parameters for our Monte Carlo simulations. We have therefore carried out parallel sets of calculations for two very different sets of heavy element abundances: the Grevesse \& Sauval (1998; GS98) abundances and the Asplund et al. (2005; AGS05) recommended abundances. We have used conservative estimates for the composition uncertainties together with the GS98 abundances and optimistic uncertainties together with the AGS05 abundances. Through- out this paper, and unless otherwise noted, we give without parentheses the results calculated with the GS98 abundances and conservative composition uncertainties and with parentheses the results calculated with AGS05 abundances and optimistic uncertainties.

Input parameters and their uncertainties. - In $\S 2$, we present and discuss the best estimates and uncertainties for 19 important input parameters that are used in constructing the solar models that are considered in this paper. These parameters include nuclear fusion cross sections, the solar age and luminosity, the diffusion coefficient, and the nine most important heavy element abundances on the surface of the Sun. Two additional input "parameters," the radiative opacity and the equation of state, are discussed separately in $\S 3$. The opacity and the equation of state are complicated functions of the local conditions in the star and must therefore be treated in a different way than the singlevalued input parameters discussed in $\S 2$. For each standard solar model we simulate, all 21 input parameters are chosen from their separate probability distributions that are described in $\S \S 2$ and 3.

Standard solar models: 23 predicted quantities and some model characteristics. - We present in $\S 5$ the best estimate predictions for 23 solar quantities that are either already measured or potentially measurable. These quantities include the eight dominant neutrino fluxes, the event rates for the chlorine and gallium solar neutrino experiments, eight quantities that have been determined precisely by helioseismological measurements, and five quantities (not all independent) that characterize the relative frequency of different nuclear fusion reactions in the Sun. We also summarize some of the main characteristics (not directly measurable) of the standard solar models, including the principal physical variables at the center of the Sun and at the base of the convective zone, as well as the initial composition. For the reader's convenience, we also present compact tables of the profile of the solar sound speed and the density. Using quadratic interpolation, these tables can be used to reproduce precise values of the sound speed and density through the Sun. Finally, we present quantities that are useful in precise analysis of solar neutrino propagation, including the radial distribution of the production of the individual neutrino sources, as well as the electron and neutron number densities as a function of solar radius.

The depth of the convective zone and the surface helium abundance.-The measured depth of the solar convective zone is in good agreement with the predictions of standard solar models constructed with the GS98 heavy element abundances (see eq. [31]). However, solar models constructed with the AGS05 recommended abundances and uncertainties ("optimistic uncertainties") disagree with the measured depth of the solar convective zone by the equivalent of $3.9 \sigma$ (see eq. [32]). The strong disagreement goes away if we adopt "conservative uncertainties" for the heavy elements together with the AGS05 abundances (see eq. [33]); this results because the optimistic uncertainties are large enough to reproduce a solar composition that resembles that of GS98.

The measured surface helium abundance is in very good agreement with solar models constructed with the GS98 heavy element abundances (see eq. [34]). The agreement is poor, however, if AGS05 abundances are used: the discrepancy between measured and predicted surface helium abundance is $3.6 \sigma$ (effective) if the AGS05 optimistic uncertainties are used and $2.8 \sigma$ (effective) if conservative uncertainties are adopted (see eqs. [35] and [36]). We conclude that the measured depth of the solar convective zone and the surface helium abundance both indicate that models constructed with the GS98 heavy element abundances are significantly closer to the actual Sun than are models 
constructed with the AGS05 recommended abundances. Figure 3 shows, for different assumed abundances and uncertainties, the distribution of solar models with different values of the depth of the convective zone and different values of the surface helium abundance.

Sound speed and density profiles.-We present in $\S 7$ the distributions of the sound speed and density difference rms. Table 12 gives the most probable values for the rms distributions and the respective uncertainties. The Monte Carlo simulation of models constructed with the GS98 composition have distributions strongly peaked very close to zero for all the six rms defined and used in this work. These distributions reflect a very good agreement between solar models constructed with the GS98 composition and results from helioseismology, reinforcing the conclusions drawn from the depth of the convective zone and surface helium abundance discussed above. The set of models constructed with the AGS05 composition, on the contrary, give rise to rms distributions that make evident the discrepancy in the sound speed and density profiles introduced by the adoption of the AGS05 composition. In addition, Figures 4 and 5 allow us to conclude that the uncertainties in all the other input parameters entering a standard solar model cannot compensate for the degradation introduced by the new recommended set of solar abundances.

The predicted solar neutrino fluxes.-We analyze in $\S 8$ the calculated distributions of solar neutrino fluxes. Table 15 gives the total $1 \sigma$ uncertainty for each neutrino source and for all three choices of heavy element composition and their uncertainties. The results are in very good agreement with the uncertainties estimated using power-law dependences of the fluxes as a function of input parameters. The distribution of calculated fluxes for each neutrino source is well described by either a normal or a lognormal distribution, depending on what is the dominant source of uncertainty, with the tabulated standard deviation.

The calculated ${ }^{8} \mathrm{~B}$ solar neutrino flux is in good agreement with the value measured by solar neutrino experiments. The theoretical uncertainty in the prediction of the ${ }^{8} \mathrm{~B}$ neutrino flux (11\%-17\%, depending on the choice of heavy element composition and uncertainties) is larger than the uncertainty $(5 \%)$ in the experimental determination. For all other solar neutrino sources, the experimental uncertainties greatly exceed the solar model uncertainties.

The ${ }^{7} \mathrm{Be}$ solar neutrino flux will be measured in the next few years. The solar model uncertainties in the prediction of the ${ }^{7} \mathrm{Be}$ neutrino flux vary from $9.3 \%$ to $10.5 \%$ depending on the choice of heavy element abundances and their uncertainties. It is possible that the pep neutrino flux will be measured in one of the same experiments as the ${ }^{7} \mathrm{Be}$ neutrino flux. The predicted anticorrelation between the pep and ${ }^{7} \mathrm{Be}$ neutrino fluxes is given in equations (40) and (41) and is shown in Figure 8.

The solar model predictions for the $p$ - $p$ and the pep neutrino fluxes are strongly correlated with each other and the $p$ - $p$ neutrino flux is anticorrelated with the ${ }^{7} \mathrm{Be}$ neutrino flux. These relations between the predicted neutrino fluxes represent important testable predictions of the solar models and are discussed and analyzed quantitatively in $\S 8$.

The correlation coefficients of the neutrino fluxes.-The correlations between the different neutrino fluxes are succinctly summarized by the matrix of correlation coefficients. Tables 16 and 17 present the correlation coefficients of the neutrino fluxes for, respectively, GS98 heavy element abundances and conservative uncertainties and AGS05 heavy element abundances and optimistic uncertainties. These correlation coefficients can be used to make a more constrained and precise analysis of solar neutrino oscillations.

Nuclear energy generation pathways.-Table 18 summarizes the calculated fraction of the total nuclear energy generation that is produced by different nuclear fusion pathways. For all choices of the surface heavy element abundances and their uncertainties, the $p$ - $p$ chain is responsible for more than $99 \%$ of the total energy generation. The estimated uncertainty in the $p$ - $p$ energy generation fraction is less than or of order $0.2 \%$. The CNO energy fraction is less than $1 \%$. About $88 \%-90 \%$ of the $p-p$ energy generation is from reactions that are terminated by the ${ }^{3} \mathrm{He}\left({ }^{3} \mathrm{He}\right.$, $2 p)^{4} \mathrm{He}$ reaction with an uncertainty of about $1 \%$. Although most of solar neutrino astronomy has so far been focused on the high-energy ${ }^{8} \mathrm{~B}$ neutrinos, the nuclear fusion reactions that lead to the production of ${ }^{8} \mathrm{~B}$ represent less than $1 \%$ of the total solar energy generation (best estimate varies from $0.81 \%$ to $0.91 \%$ with an uncertainty of only $0.08 \%$ ).

Future solar neutrino experiments that measure different solar neutrino fluxes can determine empirically the nuclear energy generation fractions and test the solar model predictions given in Table 18 .

The results presented in this work are a corollary of the continuous effort John Bahcall had put for about 40 years in studying and understanding the Sun. This paper took shape and was written by John during April and May, before most of the calculations were done (see $\S 1.2$ ). Most results were already incorporated in it by July. John Bahcall passed away on 2005 August 17. With great pain, A. M. S. and S. B. finished the preparation of the paper, particularly $\S 7$, during October. John will be deeply missed.

J. N. B. and A. M. S. were supported in part by NSF grants PHY-0070928 and PHY-0503584 to the Institute for Advanced Study. A. M. S. was also supported by the W. M. Keck Foundation through a grant-in-aid to the Institute for Advanced Study and by the Association of Members of the Institute for Advanced Study. S. B. was partially supported by NSF grants ATM-0206130 and ATM-0348837. This work utilizes data from the Solar Oscillations Investigation/Michelson Doppler Imager (SOI/MDI) on the Solar Heliospheric Observatory $(\mathrm{SOHO})$. The MDI project is supported by NASA grant NAG58878 to Stanford University. SOHO is a project of international cooperation between ESA and NASA. We are grateful to M. Chen, P. Goldreich, E. Lisi, A. McDonald, M. Pinsonneault, and S. Tremaine, and particularly to C. Peña-Garay for valuable comments, discussions, and suggestions during the extended period in which this project was carried out and the results analyzed. Computations presented in this paper were done with the Scheides Beowulf cluster at the Institute for Advanced Study.

\section{APPENDIX}

\section{LOGNORMAL DISTRIBUTION}

We briefly summarize some important properties of lognormal probability distribution functions used in this work. We also define the $1 \sigma$ confidence level interval we have adopted throughout this paper when a given quantity is lognormally distributed. 
A random variable $x$ is lognormally distributed when its probability density function $f(x)$ is given by

$$
f(x)=[s \sqrt{2 \pi}(x-\theta)]^{-1} \exp \left[-\frac{(\log (x-\theta)-m)^{2}}{2 s^{2}}\right] .
$$

Here $m$ and $s$ are the scale and shape parameters, respectively. The quantity $\theta$ is the location parameter, which we assume equal to 0 for simplicity. The most probable value (mode) and the mean values of a lognormally distributed quantity, respectively, are

$$
Q=\exp \left(m-s^{2}\right) ; \quad \mu=\exp \left(m+s^{2} / 2\right) .
$$

In general, we adopt as the $1 \sigma$ confidence level interval that given by the limits of the integral

$$
\int_{x_{-}}^{x_{+}} f(x) d x=0.683, \text { with } f\left(x_{-}\right)=f\left(x_{+}\right) .
$$

Equation (A3) uniquely defines the $1 \sigma$ confidence level interval $\left[x_{-}, x_{+}\right]$. An interesting relation between $x_{-}$and $x_{+}$is

$$
x_{-} x_{+}=Q^{2} .
$$

The $1 \sigma$ confidence level interval is conveniently expressed with respect to the mode $Q$ by introducing the quantities $\sigma_{+}$and $\sigma_{-}$ defined by

$$
\sigma_{+}=x_{+}-Q ; \quad \sigma_{-}=Q-x_{-} .
$$

When relative values for $\sigma_{+}$or $\sigma_{-}$are quoted in this paper, they are effectively calculated as $\sigma_{+} / Q$ and $\sigma_{-} / Q$.

In practice, for a given data set $\left\{x_{i}\right\}_{i=1}^{N}, m$ and $s$ are estimated as

$$
m=N^{-1} \sum_{i=1}^{N} \log x_{i}
$$

and

$$
s^{2}=(N-1)^{-1} \sum_{i=1}^{N}\left(\log x_{i}-m\right)^{2} .
$$

Abdurashitov, J. N., et al. 2002, J. Exp. Theor. Phys., 95, 181 . 2003, Nucl. Phys. B, 118, 39

Adelberger, E. G., et al. 1998, Rev. Mod. Phys., 70, 1265

Aharmim, B., et al. 2005, preprint (nucl-ex/0502021)

Ahmed, S. N., et al. 2004, Phys. Rev. Lett., 92, 181301

Alexander, D. R., \& Ferguson, J. W. 1994, ApJ, 437, 879

Alimonti, G., et al. 2002, Astropart. Phys., 16, 205

Allende Prieto, C., Lambert, D. L., \& Asplund, M. 2001, ApJ, 556, L63 2002, ApJ, 573, L137

Altmann, G., et al. 2005, Phys. Lett. B, 616, 174

Angulo, C., \& Descouvemont, P. 2001, Nucl. Phys. A, 690, 755

Antia, M., \& Basu, S. 2005, ApJ, 620, L129

Araki, T., et al. 2005, Phys. Rev. Lett., 94, 081801

Asplund, M. 2000, A\&A, 359, 755

Asplund, M., Grevesse, N., \& Sauval, A. J. 2005, in ASP Conf. Ser. 336, Cosmic Abundances as Records of Stellar Evolution and Nucleosynthesis, ed. T. G. Barnes III, \& F. N. Bash (San Francisco: ASP), 25

Asplund, M., Grevesse, N., Sauval, A. J., Allende Prieto, C., \& Kiselman, D. 2004, A\&A, 417, 751

Asplund, M., Nordlund, A., Trampedach, R., \& Stein, R. F. 2000, A\&A, 359, 743

Badnell, N. R., Bautista, M. A., Butler, K., Delahaye, F., Mendoza, C., Palmeri, P., Zeippen, C. J., \& Seaton, M. J. 2005, MNRAS, 360, 458

Bahcall, J. N. 1964, Phys. Rev. Lett., 12, 300 1989, Neutrino Astrophysics (Cambridge: Cambridge Univ. Press)

1994, Phys. Rev. D, 49, 3923

1997, Phys. Rev. C, 56, 3391

2002, Phys. Rev. C, 65, 025801

Bahcall, J. N., Bahcall, N. A., \& Shaviv, G. 1968, Phys. Rev. Lett., 20, 1209

Bahcall, J. N., Basu, S., Pinsonneault, M. H., \& Serenelli, A. M. 2005a, ApJ, 618,1049

Bahcall, J. N., Basu, S., \& Serenelli, A. M. 2005b, ApJ, 631, 1281

\section{REFERENCES}

Bahcall, J. N., Gonzalez-Garcia, M. C., \& Peña-Garay, C. 2004a, J. High Energy Phys., 8, 16

Bahcall, J. N., Krastev, P. I., \& Smirnov, A. Yu. 2001a, Phys. Rev. D, 63, 053012

Bahcall, J. N., \& May, R. M. 1969, ApJ, 155, 501

Bahcall, J. N., \& Pinsonneault, M. H. 1995, Rev. Mod. Phys., 67, 781 2004, Phys. Rev. Lett., 92, 121301 (BP04)

Bahcall, J. N., Pinsonneault, M. H., \& Basu, S. 2001b, ApJ, 555, 990

Bahcall, J. N., Pinsonneault, M. H., Basu, S., \& Christensen-Dalsgaard, J. 1997, Phys. Rev. Lett., 78, 171

Bahcall, J. N., \& Serenelli, A. M. 2005, ApJ, 626, 530

Bahcall, J. N., Serenelli, A. M., \& Basu, S. 2005c, ApJ, 621, L85

Bahcall, J. N., Serenelli, A. M., \& Pinsonneault, M. H. 2004b, ApJ, 614, 464

Bahcall, J. N., \& Ulrich, R. K. 1988, Rev. Mod. Phys., 60, 297

Barger, V., Deshpande, N., Pal, P. B., Phillips, R. J. N., \& Whisnant, K. 1991, Phys. Rev. D, 43, R1759

Basu, S. 1998, MNRAS, 298, 719

- 2002, in Proc. SOHO 11 Symp., From Solar Min to Solar Max: Half a Solar Cycle with $\mathrm{SOHO}$, ed. A. Wilson (ESA SP-508; Noordwijk: ESA), 7 Basu, S., \& Antia, H. M. 1997, MNRAS, 287, 189 2004, ApJ, 606, L85

Basu, S., Bahcall, J. N., \& Pinsonneault, M. H. 2000, ApJ, 529, 1084

Bertello, L., et al. 2000, ApJ, 535, 1066

Boothroyd, A. I., \& Sackmann, I.-J. 2003, ApJ, 583, 1004

Christensen-Dalsgaard, J., Gough, D. O., \& Thompson, M. J. 1991, ApJ, 378, 413

Cleveland, B. T., Daily, T., Davis, R., Jr., Distel, J. R., Lande, K., Lee, C. K., Wildenhain, P. S., \& Ullman, J. 1998, ApJ, 496, 505

Couvidat, S., Turck-Chièze, S., \& Kosovichev, A. G. 2003, ApJ, 599, 1434

Davis, R., Jr., Harmer, D. S., \& Hoffmann, K. C. 1968, Phys. Rev. Lett., 20, 1205

Dolbeau, J., Giomataris, I., Gorodetzky, P., Patzak, T., Salin, P., \& Sarat, A. 2005, Nucl. Phys. B, 138, 94 
Ejiri, H., Engel, J., Hazama, R., Krastev, P., Kudomi, N., \& Robertson, R. G. H. 2000, Phys. Rev. Lett., 85, 2917

Ferguson, J. W., Alexander, D. R., Allard, F., Barman, T., Bodnarik, J. G., Hauschildt, P. H., Heffner-Wong, A., \& Tamanai, A. 2005, ApJ, 623, 585 Fogli, G. L., \& Lisi, E. 1995, Astropart. Phys., 3, 185

Fogli, G. L., Lisi, E., Marrone, A., Montanino, D., \& Palazzo, A. 2002, Phys. Rev. D, 66, 053010

Formicola, A., et al. 2004, Phys. Lett. B, 591, 61

Freedman, S. J., \& Kayser, B. 2004, preprint (physics/0411216)

Friedland, A., Lunardini, C., \& Peña-Garay, C. 2004, Phys. Lett. B, 594, 347

Fröhlich, C., \& Lean, J. 1998, Geophys. Res. Lett., 25, 4377

Fukuda, S., et al. 2001, Phys. Rev. Lett., 86, 5651 2002, Phys. Lett. B, 539, 179

Fukuda, Y., et al. 1996, Phys. Rev. Lett., 77, 1683

Galbiati, C., Pocar, A., Franco, D., Ianni, A., Cadonati, L., \& Schönert, A. 2005, Phys. Rev. C, 71, 055805

Gorodetsky, P., Patzak, T., Seguinot, J., Vanel, J. C., Ypsilantis, T., Derre, J., Giomataris, I., \& Zaccone, H. 1999, Nucl. Instrum. Methods Phys. Res., 433, 554

Grevesse, N. 1984, Phys. Scr., 8, 49

Grevesse, N., \& Sauval, A. J. 1998, Space Sci. Rev., 85, 161

Gribov, V. N., \& Pontecorvo, B. M. 1969, Phys. Lett. B, 28, 493

Gruzinov, A. V., \& Bahcall, J. N. 1997, ApJ, 490, 437

Guenther, D. B., Demarque, P., Kim, Y.-C., \& Pinsonneault, M. H. 1992, ApJ, 387,372

Guenther, D. B., Kim, Y.-C., \& Demarque, P. 1996, ApJ, 463, 382

Guzik, J. A., \& Cox, A. N. 1993, ApJ, 411, 394

Guzik, J., \& Watson, L. S. 2004, in Proc. SOHO 14th-GONG 2004 Workshop, ed. D. Dansey (ESA SP-559; Noordwijk: ESA), 456

Guzik, J. A., Watson, L. S., \& Cox, A. N. 2005, ApJ, 627, 1049

Hampel, W., et al. 1999, Phys. Lett. B, 447, 127

Iglesias, C. A., \& Rogers, F. J. 1996, ApJ, 464, 943

Junghans, A. R., et al. 2003, Phys. Rev. C, 68, 065803

Junker, M., et al. 1998, Phys. Rev. C, 57, 2700

Kosovichev, A. G., \& Fedorova, A. V. 1991, Soviet Astron., 35, 507

Lanou, R. E. 2005, Nucl. Phys. B, 138, 98

Lim, C. S., \& Marciano, W. 1988, Phys. Rev. D, 37, 1368

Lodders, K. 2003, ApJ, 591, 1220

McDonald, A. B. 2004, New J. Phys., 6, 121

McKinsey, D. N., \& Coakley, K. J. 2005, Astropart. Phys., 22, 355

McKinsey, D. N., \& Doyle, J. M. 2000, J. Low Temp. Phys., 118, 153

Mikheyev, S. P., \& Smirnov, A. Y. 1985, Soviet J. Nucl. Phys., 42, 913
Mikheyev, S. P., \& Smirnov, A. Y. 1986, in '86 Massive Neutrinos in Astrophysics and in Particle Physics, Proc. of the Sixth Moriond Workshop, ed. O. Fackler \& Trân Thanh Vân (Gif-sur-Yvette: Editions Frontières), 355

Montalbán, J., Miglio, A., Noels, A., Grevesse, N., \& di Mauro, M. P. 2004, in Proc. SOHO 14th GONG 2004 Workshop, ed. D. Dansey (ESA SP-559; Noordwijk: ESA), 574

Mukhamedzhanov, A. M., et al. 2003, Phys. Rev. C, 67, 065804

Nakahata, M. 2001, in Proc. Third International Workshop on Neutrino Oscillations and Their Origin (NOON2001), ed. Y. Suzuki, M. Nakahata, Y. Fukuda, Y. Takeuchi, T. Mori, \& T.Yoshida (Singapore: World Scientific), 92 Oberauer, L., von Feilitzsch, F., \& Potzel, W. 2005, Nucl. Phys. B, 138, 108 Park, T. S., et al. 2003, Phys. Rev. C, 67, 055206

Pijpers, F. P., \& Thompson, M. J. 1992, A\&A, 262, L33 1994, A\&A, 281, 231

Proffitt, C. R. 1994, 425, 849

Rabello-Soares, M. C., Basu, S., \& Christensen-Dalsgaard, J. 1999, MNRAS, 309,35

Raghavan, R. S. 1976, Phys. Rev. Lett., 37, 259

. 2001, preprint (hep-ex/0106054)

Rogers, F. J. 2001, Contrib. Plasma Phys., 41, 179

Rogers, F. J., Swenson, F. J., \& Iglesias, C. A. 1996, ApJ, 456, 902

Runkle, R. C., et al. 2005, Phys. Rev. Lett., 94, 082503

Sackmann, I.-J., Boothroyd, A. I., \& Fowler, W. A. 1990, ApJ, 360, 727

Schou, J., Christensen-Dalsgaard, J., Howe, R., Larsen, R. M., Thompson, M. J., \& Toomre, J. 1998, in Structure and Dynamics of the Interior of the Sun and Sun-like Stars, ed. S. G. Korzennik \& A. Wilson (ESA SP-418; Noordwijk: ESA), 845

Seaton, M. J. 2005, MNRAS, 362, L1

Seaton, M. J., \& Badnell, N. R. 2004, MNRAS, 354, 457

Singh, B. S., Hass, M., Nir-El, Y., \& Haquin, G. 2004, Phys. Rev. Lett., 93, 262503

Spiro, M., \& Vignaud, D. 1990, Phys. Lett. B, 242, 279

Suzuki, Y. 2005, Nucl. Phys. B, 143, 27

Suzuki, Y., et al. 2000, preprint (hep-ph/0008296)

Thoul, A. A., Bahcall, J. N., \& Loeb, A. 1994, ApJ, 421, 828

Turck-Chièze, S., Cahen, S., Casse, M., \& Doom, C. 1988, ApJ, 335, 415

Turck-Chièze, S., Couvidat, S., Piau, L., Ferguson, J., Lambert, P., Ballot, J., García, R. A., \& Nghiem, P. 2004, Phys. Rev. Lett., 93, 211102

Turck-Chièze, S., \& Lopes, I. 1993, ApJ, 408, 347

Weiss, A., \& Schlattl, H. 2000, A\&AS, 144, 487

Wolfenstein, L. 1978, Phys. Rev. D, 17, 2369 